Linköping studies in science and technology.

Dissertation, No. 1614

\title{
MEDICAL VOLUME VISUALIZATION BEYOND SINGLE VOXEL VALUES
}

Stefan Erik Lindholm

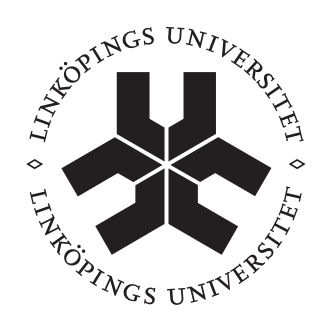

\section{Linköping University INSTITUTE OF TECHNOLOGY}

\author{
Division of Media and Information Technology \\ Department of Science and Technology \\ Linköping University, SE-601 74 Norrköping, Sweden \\ Norrköping, October 2014
}

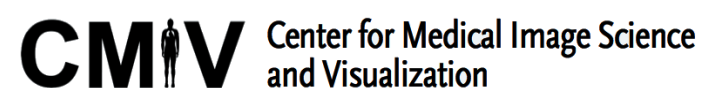

Center for Medical Image Science and Visualization (CMIV)

Linköping University Hospital, SE-581 85 Linköping, Sweden 
Medical Volume Visualization Beyond Single Voxel Values

Copyright (C) 2014 Stefan Lindholm (unless otherwise noted)

Division of Media and Information Technology

Department of Science and Technology

Campus Norrköping, Linköping University

SE-601 74 Norrköping, Sweden

ISBN: 978-91-7519-256-7

ISSN: 0345-7524

Printed in Sweden by LiU-Tryck, Linköping, 2014 


\section{Acknowledgments}

I have had the great pleasure of working with many skilled and dedicated people during my phd. Yet two people deserve extra recognition for their unending support. These people are my supervisor Anders Ynnerman and my wife Theresa Lindholm.

Thank you!

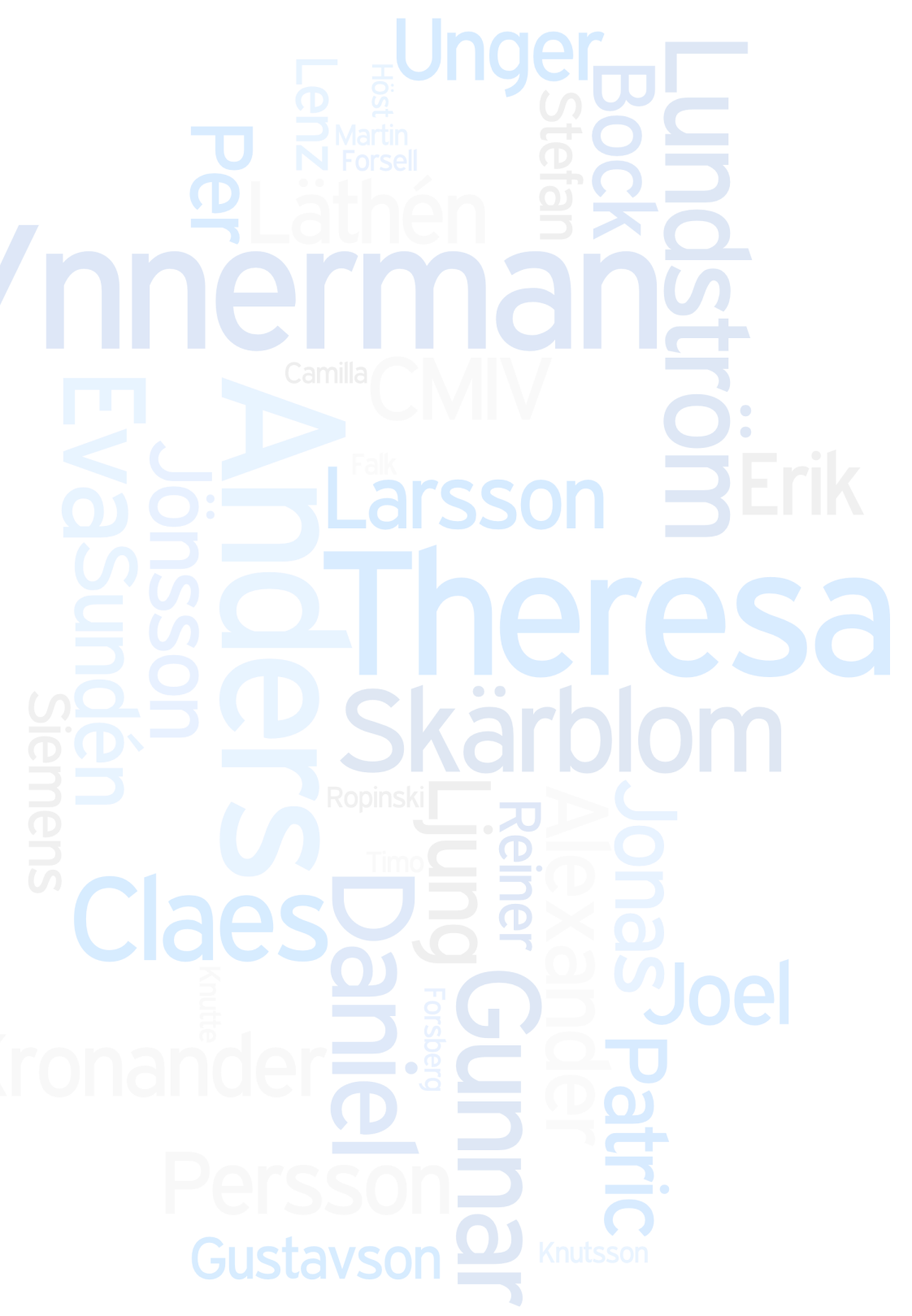


"Det handlar om att tänka lite mer och koda lite mindre." 


\section{Abstract}

Medical visualization involves many complex decisions for both the user and the imaging algorithms. This thesis aims to improve medical volume visualization through a series of technical contributions to aid such decision processes. Improvements are achieved by using more data, beyond single voxels, in the associated visual analyses.

Simultaneous visualization of multiple data sources and different data formats is rapidly becoming a necessity. This is due to both the growing number of data producing image acquisition techniques as well as the increase in geometric data representations that can be created. Maintaining high rendering performance under these circumstances is challenging, but necessary, to support an exploratory visualization process. This thesis proposes two algorithms to address this challenge: a multi-volume approach that applies binary-space partitioning to solve painters' algorithm geometrically and a rendering algorithm for hybrid data that improves the management of the available graphics memory.

Additional information for decision support is often derived from the captured image data. Classification techniques, in particular, often utilize secondary information sources or neighborhood analysis as means to improve specificity. One example is a proposed algorithm that improves visualization of blood vessels by automatically optimizing visualization parameters based on observed vesselness. This thesis also proposes algorithms involving neighborhood analysis, with a particular focus on domain specific classification knowledge provided by the user. One algorithm provides the ability to semantically state spatial relations between tissues based on encoded material information. Another algorithm improves the representation of discrete features by integrating the users' knowledge in the reconstruction step of the visualization pipeline.

Many of the methods proposed in this thesis can also be applied to other domains, but are all described here in the context of medical volume visualization as most of the research has been performed within this field. 


\section{Populärvetenskaplig Sammanfattning}

Medicinsk visualisering involverar en mängd komplexa beslut, både för användaren och för bildsystemet. I många fall handlar dessa beslut om vilken information som skall visas och hur den skall visas. Utfallen av dessa beslut kan potentiellt förbättras ju mer data som inkluderas i beslutsprocessen bortom enskilda voxlar. Den här avhandlingen avser att förbättra och effektivisera medicinsk volumsvisualisering genom en rad tekniska forskningsbidrag.

Samtidig visualisering av multipla datakällor och olika dataformat är snabbt på väg att bli en nödvändighet. Dels på grund av det växande antalet dataproducerande bildmetoder, men även på grund av det stigande antalet geometriska representationer som kan skapas. Att producera snabba effektiva renderingar under dessa förutsättningar är svårt, men också nödvändigt för att stödja den ofta explorativa visualiseringsprocessen. Ytterligare information för beslutstagande härleds även från existerande bilddata. Speciellt klassificeringstekniker använder ofta data från sekundära källor eller analyser av lokala regioner för att till exempel bättre kunna skilja olika vävnader åt. Ett exempel är en metod som föreslås i denna avhandling vilken automatiskt optimerar visualiserinsparametrar med avseende på ett mått om hur mycket kärl som förekommer i den renderade bilden. Denna avhandling presenterar även metoder baserade på analys av lokala dataregioner, med ett speciellt fokus på domänspecifik klassificeringskunskap som tillhandahållits av användaren själv.

Flera av de metoder som presenteras i denna avhandling är även applicerbara inom andra domäner men är samtliga presenterade här i kontexten av medicinsk volymsvisualisering då majoriteten av forskningen utförts inom detta område. 


\section{Publications}

The following publications are included in the dissertation:

Paper I: S. Lindholm, P. Ljung, M. Hadwiger, and A. Ynnerman. Fused multivolume DVR using binary space partitioning. Computer Graphics Forum, 28(3):847-854, 2009. doi:10.1111/j.1467-8659.2009.01465.x.

Paper II: S. Lindholm, M. Falk, E. Sundén, A. Bock, A. Ynnerman, and T. Ropinski. Hybrid data visualization based on depth complexity histograms. Computer Graphics Forum, 2014. doi:10.1111/cgf.12460, In press.

Paper III: G. Läthén, S. Lindholm, R. Lenz, A. Persson, and M. Borga. Automatic tuning of spatially varying transfer functions for blood vessel visualization. IEEE Transactions on Visualization and Computer Graphics, 18(12):2345-2354, 2012. doi:10.1109/TVCG.2012.203.

Paper IV: G. Läthén, S. Lindholm, R. Lenz, and M. Borga. Evaluation of transfer function methods in direct volume rendering of the blood vessel lumen. In Eurographics Workshop on Visual Computing for Biology and Medicine (VCBM), Vienna, Austria, 2014. The Eurographics Association, In press.

Paper V: S. Lindholm, D. Forsberg, A. Ynnerman, H. Knutsson, M. Andersson, and C. Lundström. Towards clinical deployment of automated anatomical regions-of-interest. In Eurographics Workshop on Visual Computing for Biology and Medicine (VCBM), Vienna, Austria, 2014. The Eurographics Association, In press.

Paper VI: S. Lindholm, P. Ljung, C. Lundström, A. Persson, and A. Ynnerman. Spatial conditioning of transfer functions using local material distributions. IEEE Transactions on Visualization and Computer Graphics, 16 (6):1301-1310, 2010. doi:10.1109/TVCG.2010.195.

Paper VII: S. Lindholm, D. Jönsson, C. Hansen, and A. Ynnerman. Boundary aware reconstruction of scalar fields. IEEE Transactions on Visualization and Computer Graphics, 20(4), 2014. doi:10.1109/TVCG.2014.2346351, In press. 
The following publications were published as part of the work related to this thesis but are not part of the dissertation:

- S. Lindholm and A. Bock. Poor man's rendering of segmented data. In SIGRAD, pages 19-54, Norrköping, Sweden, 2013. [64]

- S. Lindholm, D. Jönsson, H. Knutsson, and A. Ynnerman. Towards coherent sampling in volume rendering using iso surfaces. In $S I G R A D$, pages 55-60, Norrköping, Sweden, 2013. [68]

- S. Lindholm and J. Kronander. Accounting for uncertainty in medical data: A CUDA implementation of normalized convolution. In SIGRAD, pages 35-42, Stockholm, Sweden, 2011. [65]

- C. Lundström, A. Persson, S. Ross, P. Ljung, S. Lindholm, F. Gyllensvärd, and A. Ynnerman. State-of-the-art of visualization in post-mortem imaging. Acta Pathologica (APMIS), 120(4):316-326, 2012. doi:10.1111/j.16000463.2011.02857.x. [78]

- S. Doyle, J. Monaco, A. Madabhushi, S. Lindholm, P. Ljung, L. Ladic, J. Tomaszewski, and M. Feldman. Evaluation of effects of JPEG2000 compression on a computer-aided detection system for prostate cancer on digitized histopathology. In IEEE International Symposium on Biomedical Imaging (ISBI), pages 1313-1316, Rotterdam, Netherlands, 2010. doi:10.1109/ISBI.2010.5490238. [16] 


\section{Contributions}

Paper I proposes advancements in the field of multi-volume fusion. The method achieves efficient and flexible fusion through utilization of a binary space partitioning data structure.

Paper II improves upon existing literature for visualization of hybrid data containing both volumetric and geometric objects. The methods yield significant performance gains through improved utilization of local cache memory. The performance increase becomes an enabling factor for realtime visualization of larger data sets.

Paper III proposes improvements to visualization of vascular trees. The method facilitates quick vessel tree overviews by automatically adjusting the transfer function to local shifts in vessel intensity. As a second author, my contribution was primarily the design of the regularization step as well as the presentation of the method in the manuscript.

Paper IV consists of an evaluation of the methods presented in Paper III. The evaluation assesses the feasibility of using locally adjusted transfer functions and their correctness in vessel lumen visualization.

Paper V investigates requirements for clinical deployment of automatic regionof-interest selection. A combination of image registration and distance based transfer functions is presented and evaluated in a qualitative study.

Paper VI introduces spatial conditioning of transfer functions based on material proximity relations for volume visualization. The method analyzes and encodes material footprints in attribute space, which are used to form semantic expressions such as "internal air" or "contrast agent inside liver".

Paper VII improves the representation of discontinuities and transitional regions in volume visualization. The method relies on user-provided classifiers, thereby avoiding costly segmentation procedures. Results include simple-to-achieve visualizations that capture the discrete nature of objects with disjunct features. 


\section{Contents}

Acknowledgments

Abstract $\quad$ v

Populärvetenskaplig Sammanfattning vi

List of Publications vii

$\begin{array}{ll}\text { Contributions } & \text { ix }\end{array}$

1 Motivation 1

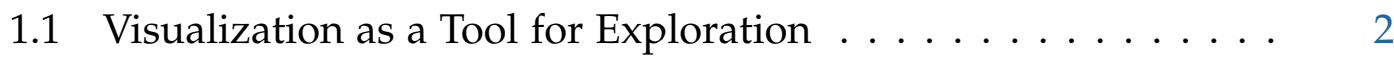

1.2 Visualization in Modern Healthcare . . . . . . . . . . . . . . . . . 2

1.3 Painter's Algorithm and Visualization . . . . . . . . . . . . 4

2 Core Aspects of Medical Visualization $\quad 7$

2.1 Short Terminology of Visualization . . . . . . . . . . . . . . . 7

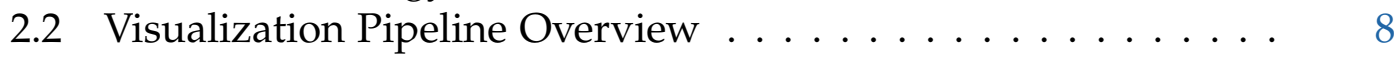

2.3 Data Acquisition . . . . . . . . . . . . . . . . 8

2.3.1 Common Data Types . . . . . . . . . . . . . . . 9

2.3 .2 Histograms . . . . . . . . . . . . . . . . . . 11

2.4 Direct Volume Rendering . . . . . . . . . . . . . . . . . . . 12

2.4 .1 The Optical Model . . . . . . . . . . . . . . . . . . . 12

2.4 .2 Computational Solvers . . . . . . . . . . . . . . . . . . 14

2.4.3 Sampling and Reconstruction . . . . . . . . . . . . 15

2.5 Fused Data Visualization . . . . . . . . . . . . . . . . . . 15

2.5.1 Multi-volume Fusion . . . . . . . . . . . . . . . . . 15

2.5 .2 Levels of Intermixing . . . . . . . . . . . . . . . . . . . . . . . . . . . . . .

2.6 Transfer Functions . . . . . . . . . . . . . . . . . . . . . . . 17

2.6.1 Pre- and Post-classified DVR . . . . . . . . . . . . 17

2.6.2 One-dimensional Transfer Functions . . . . . . . . . . . . . . 17

2.6.3 Multi-dimensional Transfer Functions . . . . . . . . . . . 18

2.6.4 Widget Based Transfer Functions . . . . . . . . . . . 18

3 Trends and Challenges in Medical Visualization 21

3.1 Challenges of Modern Healthcare . . . . . . . . . . . . . . . . . 21

3.225 Years of Visualization . . . . . . . . . . . . . 22

3.3 Advancements in Fused Data Visualization . . . . . . . . . . . . 24

3.3.1 Geometry Based Approaches To Multi-volume Fusion . 24 
3.3.2 Depth Peeling and the Transition to the GPU . . . . . . . 25

3.3.3 A-buffers and the Inclusion of Geometry . . . . . . . . . 25

3.4 Transfer Functions $2.0 \ldots \ldots \ldots$. . . . . . . . . . . . 27

3.4.1 Classification Specificity and Multiple Dimensions . . . . 27

3.4 .2 Explicit Spatial Information . . . . . . . . . . . . . . 28

3.4.3 High-level Semantics and Work Flow . . . . . . . . . . 29

3.5 Visualization of Vessels . . . . . . . . . . . . . . . . . 30

3.5.1 Vesselness Measures and Transfer Functions . . . . . . . . 30

3.5.2 Centerlines and Segmentation . . . . . . . . . . 30

3.6 Discontinuities and Partial Volume . . . . . . . . . . . . . 31

3.6.1 Visual Classification ................ . . 32

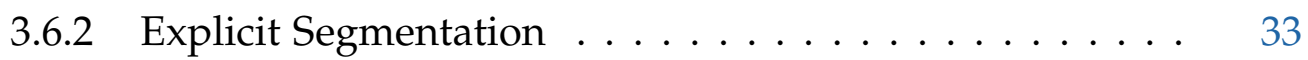

3.6 .3 Statistical Models . . . . . . . . . . . . . . . 33

4 Medical Volume Visualization Beyond Single Voxel Values 37

4.1 Overview of Contributions . . . . . . . . . . . . . . . 37

4.2 Consolidation of Multiple Data Sources . . . . . . . . . . . . . 38

4.2.1 Binary Space Partitioning for Volumetric Fusion . . . . . 38

4.2.2 Per-Pixel Depth Complexity Adjustments for Hybrid Data 40

4.3 Feature Prediction and Data Prioritization . . . . . . . . . . . 44

4.3.1 Automatic Transfer Function Tuning Based on Vesselness 44

4.3.2 Registration Based Regions-of-Interest . . . . . . . . . . 46

4.4 Probabilistic Classifiers and User Knowledge . . . . . . . . . . . . 48

4.4.1 Clinical Data Prioritization by Spatial Conditioning . . . 49

4.4.2 Improved Representations of Discontinuities . . . . . . . 52

5 Generalizations and Reflections $\quad 59$

5.1 Transfer Functions $3.0 \ldots \ldots$. . . . . . . . . . . . . . . 59

5.1.1 Problematizing Transfer Function Classification . . . . . . 60

5.1.2 Complications of Interpreting User Input . . . . . . . . . . . . 61

5.1.3 Problematizing Material Probability ........... 62

5.2 Summary and Future Work . . . . . . . . . . . . . . . 63

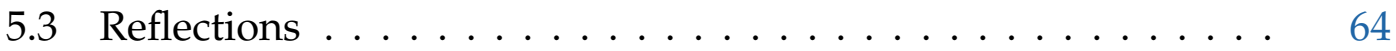

$\begin{array}{ll}\text { Bibliography } & 67\end{array}$ 



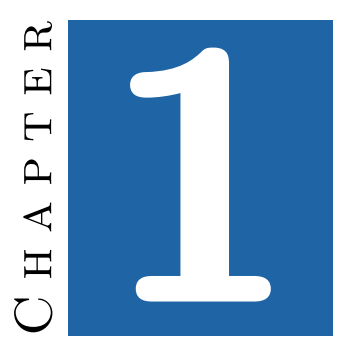

Motivation

The art of putting complex information into visible form-to visualize-has been employed for centuries. One example is the drawings penned in the notebooks of Leonardo Da Vinci, exemplified through the sketch "view of a skull" in Figure 1.1 (dated ca. 1489). Da Vinci made many similar sketches throughout his life, portraying his findings as he explored the human body and the world around him. Yet the process that led to Da Vinci's drawings does not fully capture what we today call visualization.

Visualization is today not only synonymous with images generated by the observer but also for the observer. What used to be a tool only for documenting and spreading existing knowledge is now also a tool for gaining new knowledge.

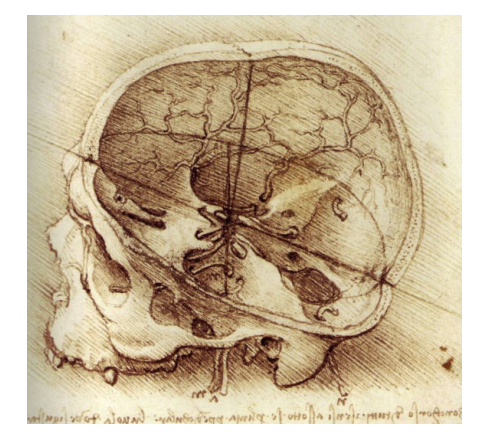

A Figure 1.1 — “View of a skull” by Leonardo Da Vinci; painted ca. 1489. 

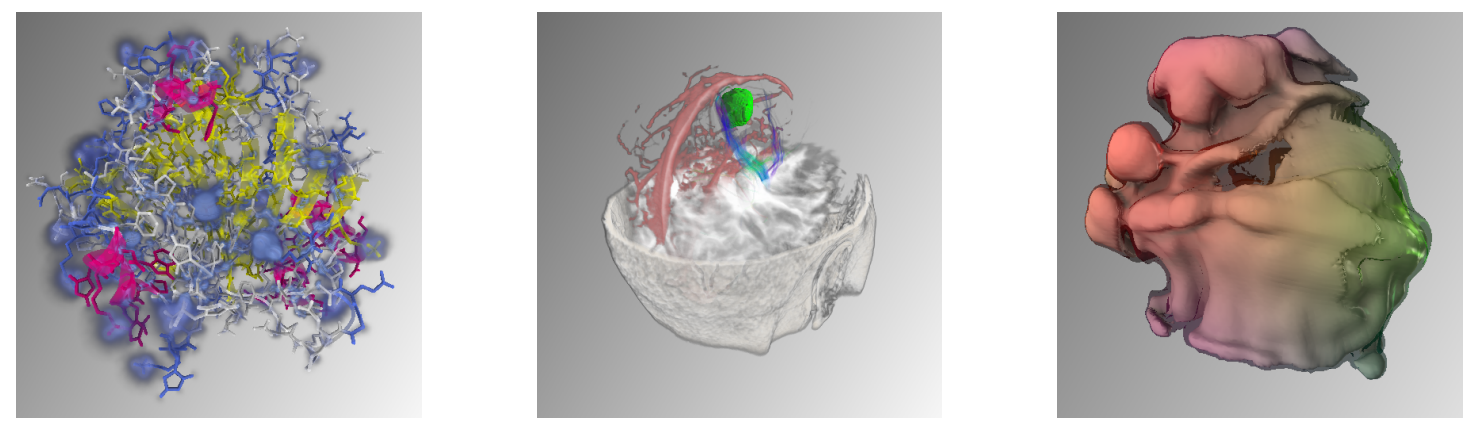

$\Delta$ Figure 1.2 - Examples of contemporary visualizations from the very small (molecular science) to the very big (space science).

\subsection{Visualization as a Tool for Exploration}

Perhaps the most fascinating use of visualization is the possibility to visually explore complex real-world data captured in domains which previously could only be artistically illustrated. This includes data of vastly different form and content, from tiny observations in the micro world to larger phenomena in the macro world. Even so, as much of the data have inherent structures, there are often specific ways in which it is most naturally explored.

Take the images in Figure 1.2 as examples. The first image (left) shows a threedimensional (3D) rendering of the human protein 'carbonic anhydrase II'. The rendering includes both geometric and volumetric data sources, respectively providing information on protein structure and charge densities. Combining these data sources in a single visualization allows the user to interact and explore the protein and the interplay of its different properties. The second image (center) shows a planning scenario for neurosurgery where some of the structures are prioritized while others are cut away to increase focus on the important neural pathways. The third image (right) shows a 3D rendering of particle density after a coronal mass ejection from the sun. Here, researchers are studying the similarities and differences among individual events to improve predictions regarding future events.

While the three examples in Figure 1.2 represent vastly different phenomena, the tools utilized for visualization and exploration are very similar.

\subsection{Visualization in Modern Healthcare}

Of particular interest to this thesis is the role of visualization in healthcare. Visualization already plays a key role in many areas of medical research and practice, and its importance is predicted to increase. To put in perspective how 

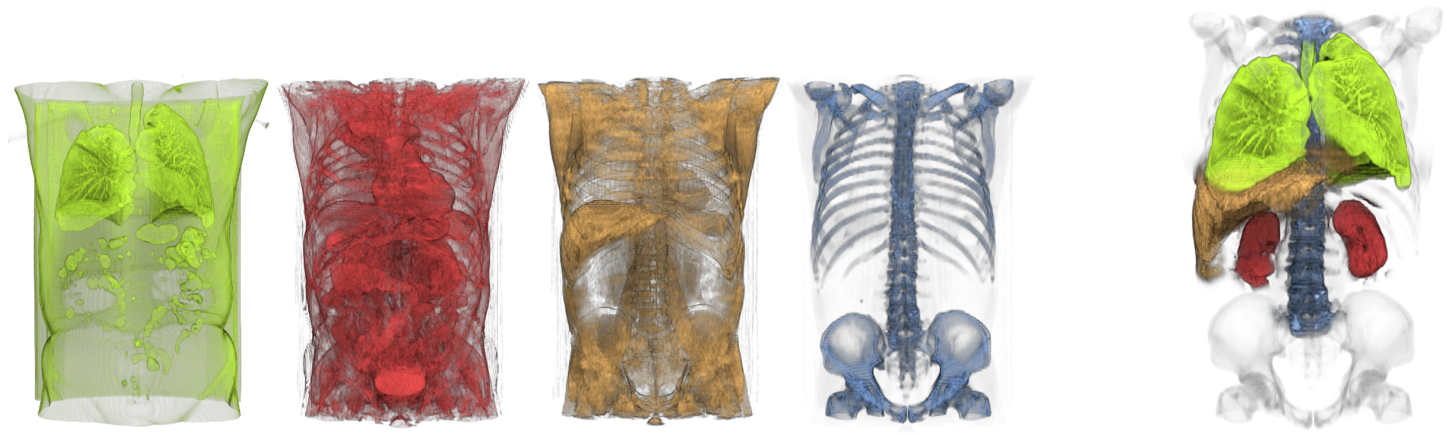

$\Delta$ Figure 1.3 - One challenge of volumetric visualization is that too much data are often shown. More useful visualizations can be created if less important data can be de-emphasized.

far visualization has already come, consider the contemporary visualization of the clinical case in Figure 1.2 (center). This visualization looks, in many ways, similar to Da Vinci's drawing from over 500 years ago (cf. Figure 1.1). Yet Da Vinci likely needed time to draw his sketch and reportedly did so often by first opening and studying his model (post-mortem). In contrast, the contemporary visualization is completed in a matter of milliseconds, using data captured with non-invasive procedures. Da Vinci was also studying human anatomy in general, whereas the majority of today's visualizations are concerned with studying the anatomy of specific patients for specific purposes.

Medical visualization is today dominated by established slice-viewing procedures where the user interacts with the data one two-dimensional (2D) image at a time. The alternative investigated in this thesis is volume visualization, where medical data is viewed in 3D, such as in the example of Figure 1.2 (center). Many of the benefits of volume visualization in the medical context can be traced to difficulties of the slice-based procedures. In two dimensions, the user often finds herself with a shortage of visual data, being forced to repeatedly scroll through stacks of images to construct a mental image of the data. For volume visualization, the situation is often the opposite; with a user forced to clip or cut the data in order to access important regions.

As an example, consider a user who intends to create a visualization including the liver, lungs, kidneys and spine. This visualization is likely to result in a combination of the first four images to the left in Figure 1.3, while the user aims for and would prefer the image on the right. The solution is as evident as it is difficult to accomplish—to only visualize the information that the user wants to see. 


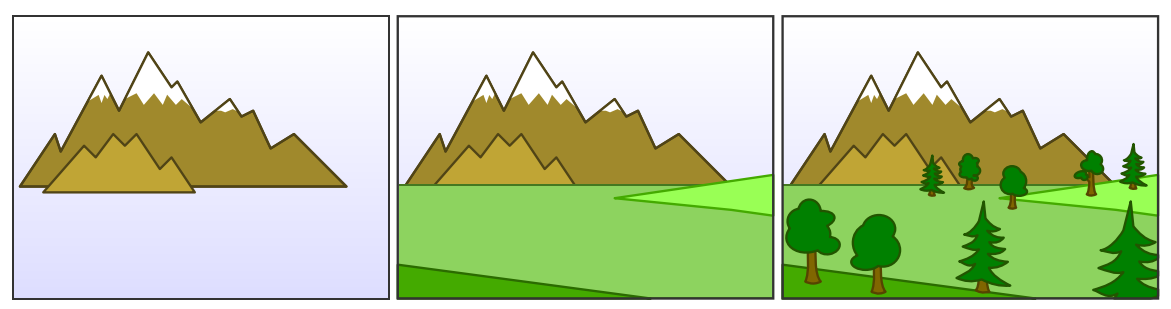

$\Delta$ Figure 1.4 - Painter's algorithm refers to the technique of painting layers of an image in order of depth. The same practice remains the core of visualization even to this day. Illustration by Zapyon, licensed under CC-SA 3.0.

\subsection{Painter's Algorithm and Visualization}

Despite immense progress, many aspects of visualization are still as important now as they were in the times of Da Vinci. One example is painter's algorithm, which refers to a solution for painting a 3D scene on a 2D canvas. The algorithm mandates that objects in the scene are painted in order from background to foreground to ensure correct occlusion, as illustrated in Figure 1.4.

While this thesis will contain many concepts and theories which may be strange or unfamiliar for an uninitiated reader, I encourage you to keep the following in mind. In its core, visualization remains very simple: find out which pieces to draw, which colors to draw them, and draw them in the correct order. The remaining 180 or so pages of this thesis are really only about finding the most important pieces and picking the best colors. 




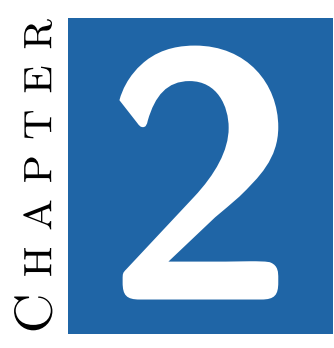

\section{Core Aspects of Medical Volume Visualization}

This chapter introduces a set of key concepts of medical visualization and Direct Volume Rendering (DVR). The presentation is not meant to be exhaustive, but rather an introduction to the field, with sufficient background information and focus on selected topics necessary for understanding the latter parts of this thesis.

For a comprehensive account of medical visualization and its aspects, readers are referred to Visualization in Medicine: Theory, Algorithms, and Applications by Preim and Bartz [92].

\subsection{Short Terminology of Visualization}

Classification and segmentation are two terms often used interchangeably in visualization community. The following quotation exemplifies their ambiguity

Segmentation means to divide up the image into a patchwork of regions, each of which is "homogeneous", that is, the "same" in some sense. Intensity, texture, colour, etc.

Classification means to assign to each point in the image a tissue class, where the classes are agreed in advance. Grey matter, White matter, Air, and so on. 
Note that the problems are inter-linked: a classifier implicitly segments an image, and a segmentation implies a classification.

Prof. Michael Brady, University of Oxford [7]

In this thesis, the use of the two terms are explicitly separated. Classification is used in reference to functions which map values or local statistics to either visual attributes or probabilistic estimates. Segmentation on the other hand is used in reference to more computationally demanding processes such as region growing or level set algorithms.

Another set of terms that are occasionally used interchangeably are interpolation and reconstruction. The latter term is preferred; as parts of the thesis involves Gaussian kernels, which are not strictly interpolative. Unless noted otherwise, the term reconstruction is always used in reference to the estimation of an off-grid signal value.

Finally, the terms tissue, material and feature are all used frequently in this thesis. The more commonplace terms, tissue and material, are preferred in earlier chapters for presentational purposes while the more generic term, feature, is preferred in later chapters.

\subsection{Visualization Pipeline Overview}

Medical visualization can be described by the flow of information from the patient up to the clinician's decision. This is outlined in the visualization pipeline, here composed of the following stages: data acquisition, data processing, image rendering, and visual analysis.

While this pipeline is traditionally linear, new research discoveries and changes in the work flow increase the interdependency between different stages. This is evidenced in an illustration provided by Birkeland et al. [6], shown in Figure 2.1. The illustration is made specifically for the ultrasound pipeline, but captures well the complexities of decision making in medical visualization.

This thesis mainly concerns the image rendering and visual analysis stages of the visualization pipeline, with a particular focus on DVR.

\subsection{Data Acquisition}

The first stage of the visualization pipeline refers to the gathering of data, or data acquisition. 


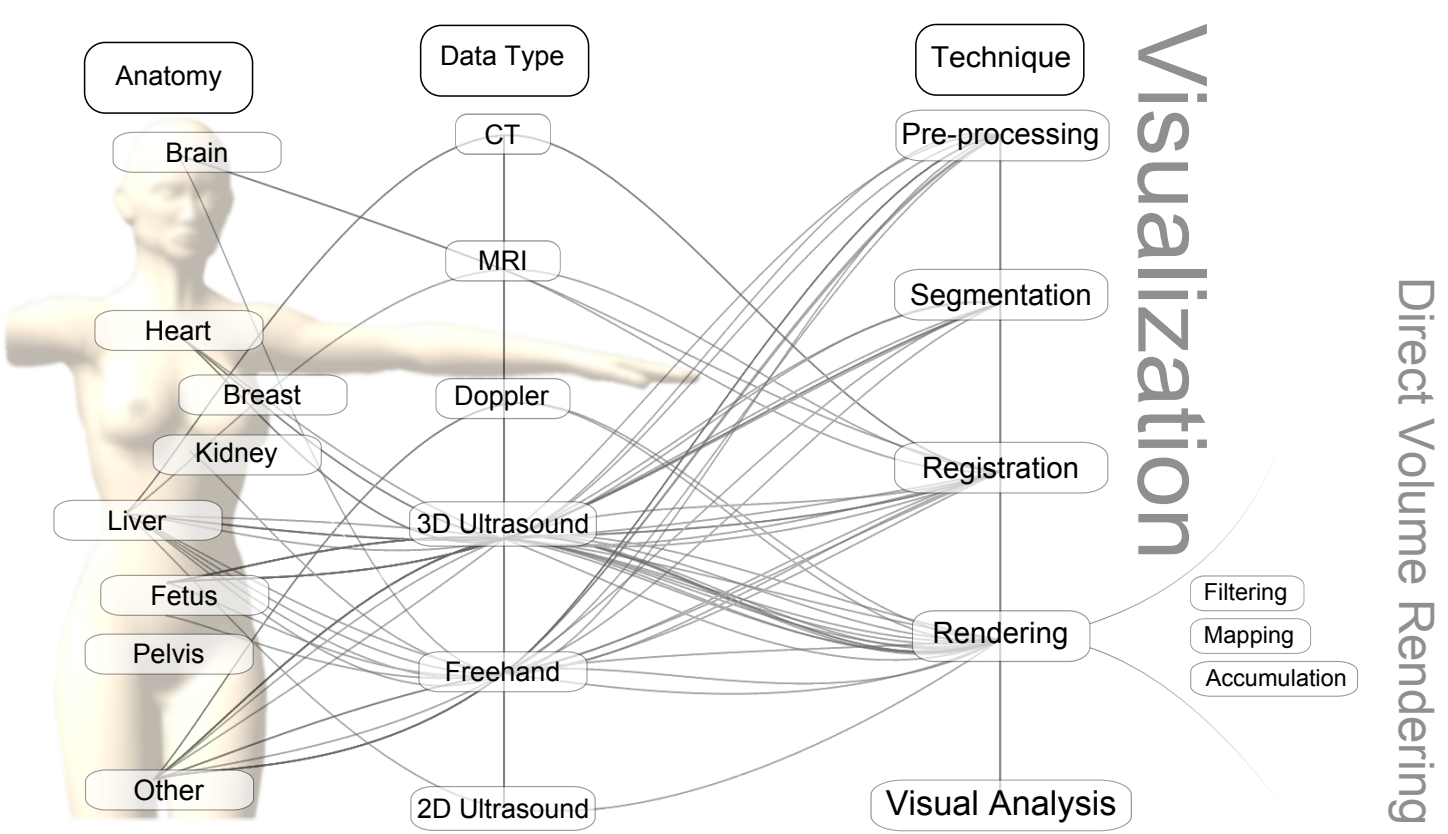

$\Delta$ Figure 2.1 - Information processing in medical visualization. Visualization pipeline: data acquisition, data processing (segmentation/registration), image rendering and visual analysis. DVR pipeline: filtering, mapping and accumulation. Original illustration created specifically for ultrasound by $\AA$. Birkeland [6]. Re-printed with permission.

\subsubsection{Common Data Types}

Described below are a number of acquisition techniques, or data types, commonly used in various medical procedures.

\section{Anatomical Imaging}

Computed Tomography (CT) is an advancement of the traditional flat x-rays. The modality produces highly detailed images and is particularly well suited for studying dense materials, such as bone or artery calcifications. The procedure is not risk free, as CT relies on tissue damaging radiation. CT is for medical purposes measured in Hounsfield units (HU) with highly predictable value ranges for known tissue types.

Magnetic Resonance Imaging (MRI) is the second of the two most common sources of volumetric information. It is preferred over CT for distinguishing softer tissues and its procedure is considered near risk free, as it relies on oscillating magnetic fields rather than radiation. Tissue value ranges are traditionally 


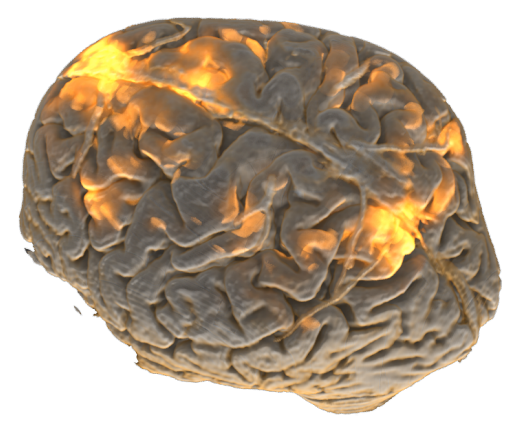

$\triangle$ Figure 2.2 - Magnetic Resonance Imaging (MRI) and functional MRI (fMRI) exemplify how anatomical and functional imaging can be used together. () K. T. Nguyen. Re-printed with permission.

less predictable for MRI than for CT due to factors such as its dependency on target temperature.

Both CT and MRI are capable of capturing time sequence data, as well as smaller focused regions at increased resolutions. Both modalities are also used in conjunction with so-called contrast agents, which are used to make certain tissues easier to distinguish. A specialized form of vessel study relying on such contrast agents is called CT Angiography (CTA). In the CTA procedure, a high density contrast agent is injected into the patent's blood flow prior to the acquisition, which highlights the vessel lumen in the resulting data.

\section{Functional Imaging}

Functional imaging is a growing field of data acquisition focusing on capturing changes in, for example, metabolism, oxygen levels and perfusion. Specific modalities include positron emission tomography (PET), single photon emission computed tomography (SPECT), perfusion imaging and functional MRI (fMRI). The latter of these is illustrated in Figure 2.2 where oxygen levels in the blood, known as the BOLD signal, are used to highlight currently active regions in the brain.

Functional imaging modalities generally tend to produce lower resolution images than anatomical imaging modalities. Functional data can be captured as an independent scan, but it is also commonly captured in the same scan procedure as the anatomical data, such as in a combined PET/CT system. Simultaneous capture is preferable, since it often eliminates the necessity to align the data in a separate post-processing stage after the scan. 

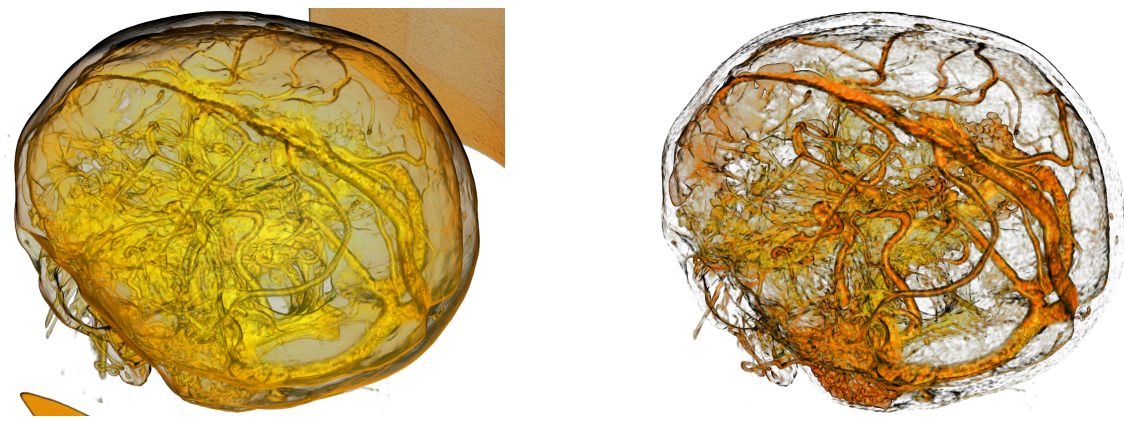

$\triangle$ Figure 2.3 - DECT exploits material differences in energy response by capturing and using two energy levels to differentiate otherwise similar looking materials. Images show CT (left) and DECT (right).

\section{Multi-source and Multi-variate Data}

As exemplified in Figure 2.1, many sources of data can be used in a single procedure. This is often referred to as multi-source data, where each modality has its own sample grid and coverage. If the data are aligned and defined on the same grid, it is instead called multi-variate.

For example, a particular form of CT called Dual-energy CT (DECT) captures two energy levels simultaneously. Although two separate x-ray tubes are used, the data consists of two values for each sample point and is therefore referred to as multi-variate.

Having more information per sample point can, for instance, be used to increase the separability between different tissues. This is illustrated in Figure 2.3 which compares renderings between single-source CT and DECT.

\subsubsection{Histograms}

One of the most frequently used tools to analyze data is the histogram. Histograms provide information on how many times different data values occur, i.e. their frequency. They are often represented graphically as bar charts, where each bar corresponds to the number of occurrences for a specific value range.

In medical visualization, histograms play a central role in detecting and categorizing materials through identifiable 'peaks' in the associated bar charts. For commonly occurring tissues, such as blood or fat, these peaks are observable directly. For less frequent tissues, the peaks may be obscured and hard to identify for a human observer, particularly if the data contains noise. This is illustrated in Figure 2.4 which shows two histograms from the same synthetic data set. In the left histogram, the three materials are clearly separated. In the 

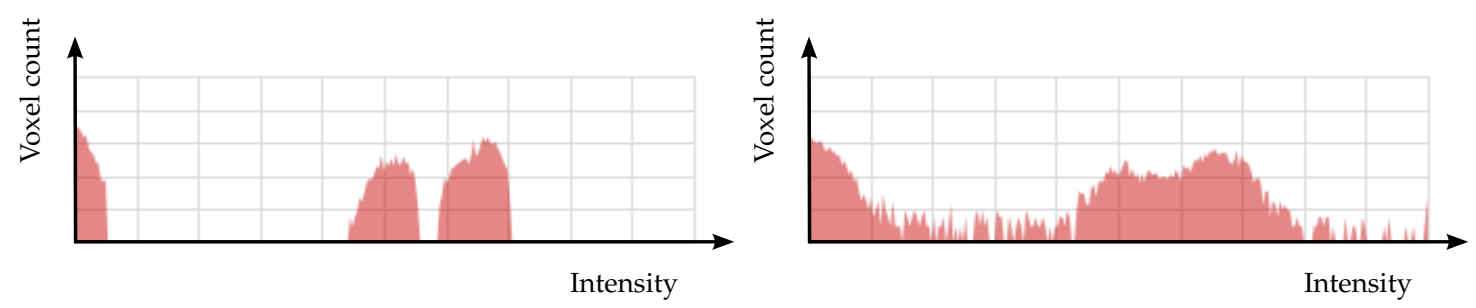

$\Delta$ Figure 2.4 - Histograms for a synthetic data set, using the original data (left) and the same data with added noise (right).

right histograms, noise has been added to the data which makes the individual materials harder to distinguish.

Histograms computed over smaller sub-regions of the data are referred to as local histograms. A key benefit of local histograms is that minor peaks which are not visible in a global histogram may still dominate a local histograms.

\subsection{Direct Volume Rendering}

Direct Volume Rendering is an important family of tools for the process of producing viewable 2D images from 3D data. The DVR rendering process is very reminiscent of painter's algorithm as it mimics the functionality of a pinhole camera, with (imaginary) light passing through the data before reaching the observer. Figure 2.5 illustrates raycasting for a single viewing ray.

The rendering process is commonly described in three steps, as highlighted in the magnified DVR pipeline to the right in Figure 2.1. First, a function value is estimated from the discrete data (data filtering). Optical properties, such as color and transparency, are then assigned based on this function value (visual mapping). Final pixel colors are then determined by sequentially analyzing the data and computing visual contributions along one viewing ray per pixel (color/opacity accumulation).

This section will look more closely at some of these aspects and introduce the concepts of reconstruction, Transfer Functions (TFs) and data fusion.

\subsubsection{The Optical Model}

The interaction between light and a participating media is often described as the net gain or loss (differential change) of radiance at any spatial location. Common terms of the applied models include emission and absorption, as well as scattering towards and away from the point of interest. For increased physical correctness, the equations should be defined for all possible light directions and 


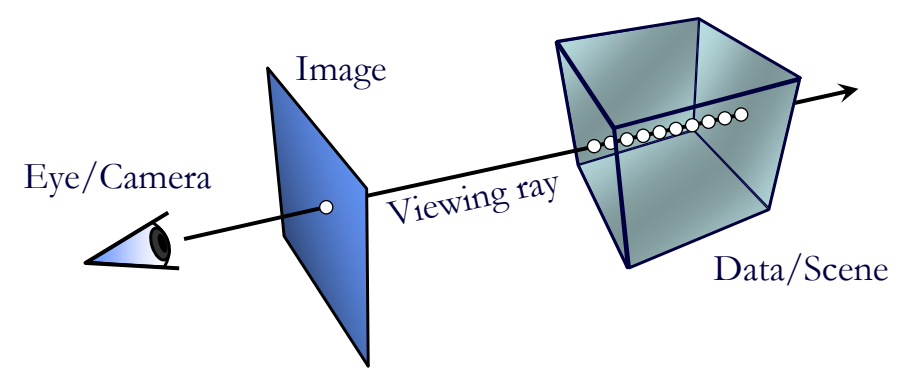

$\Delta$ Figure 2.5 - Direct Volume Rendering (DVR) is the process of creating 2D images (blue square) from 3D data (teal cube) by tracing rays from the observer through the scene. Original illustration by Engel et al. [19].

include all dominant sources of radiance. Such models are unfortunately often very expensive and unpractical. A common trade-off utilized in DVR is to limit the differential expression to two terms: emission $q$ and absorption $\kappa$. Another simplification is that the model is considered only for a single direction along the viewing ray. With the viewing ray parameterized by $\lambda$, the simplified one dimensional radiance differential can be written as

$$
\frac{\mathrm{d} I}{\mathrm{~d} \lambda}=\kappa(\lambda) I(\lambda)-q(\lambda)
$$

which solution when solved for $I$ is

$$
I(D)=\int_{0}^{D} q(\lambda) e^{-\int_{0}^{\lambda} \kappa(t) \mathrm{d} t} \mathrm{~d} \lambda
$$

The expression in Equation 2.2 forms the so-called volume rendering integral. It is presented here in its front-to-back form with the ray parameterized in the same direction. The expression also assumes that there is no background intensity.

For efficient evaluation, Equation 2.2 is partitioned to a large number of small segments $i \in[0, n]$. The following simplifications are also made within each segment: emission is modeled as a function of absorption, $q(\lambda) \approx c(\lambda) \kappa(\lambda)$, both color and absorption are assumed constant, $c(\lambda) \kappa(\lambda) \approx c_{i} \kappa_{i}$, and the resulting transparency expression is approximated by a Taylor expansion, $e^{-\kappa_{i} \Delta \lambda} \approx 1-$ $\kappa_{i} \Delta \lambda$. Between the partitioning and simplifications, the integral in Equation 2.2 is reduced to the following expression

$$
I(D) \approx \sum_{i=0}^{n}\left(c_{i} \kappa_{i} \Delta x \prod_{j=0}^{i}\left(1-\kappa_{j} \Delta x\right)\right) .
$$


Equation 2.3 is, in turn, expressed as a set of blending operations that incrementally solves the integral along a single viewing ray

$$
\begin{aligned}
& C_{\mathrm{dst}} \leftarrow C_{\mathrm{dst}}+\left(1-\alpha_{\mathrm{dst}}\right) C_{\mathrm{src}} \\
& \alpha_{\mathrm{dst}} \leftarrow \alpha_{\mathrm{dst}}+\left(1-\alpha_{\mathrm{dst}}\right) \alpha_{\mathrm{src}} .
\end{aligned}
$$

The presentation of the derivation from the radiance differential (Equation 2.1) to the blending equations (Equation 2.4) in this work is very brief and many notable assumptions are not discussed in detail. For the interested reader, there are extensive literature that includes both the above derivation and its alternatives [20, 22, 38, 40, 63, 84].

Unfortunately, notation differs across the literature and few intermediate derivation steps are provided. Inquisitive readers are encouraged to begin with the work of Max [84] and note that the derivation from Equation 2.1 to 2.2 involves the product rule, the chain rule and the second theorem of calculus. Another aspect often left out in the literature is that front-to-back and back-to-front variations of Equation 2.2 can be derived by inverting the initial differential.

\subsubsection{Computational Solvers}

Raycasting, which is illustrated in Figure 2.5, is arguably the most common way to computationally solve the discrete DVR problem in Equation 2.3. In short, one ray for each pixel of the rendered image is traversed through the scene as a loop over samples in the view direction. Entry and exit points are computed as the first and last intersections between the ray and the scene. This ensures that only those parts of the ray that intersect the volume are rendered. At all sample points along the ray, the data are sampled and transformed into visual contributions that are blended to the total color and transparency of the pixel, according to Equation 2.4. Raycasting implementations are often intuitive in relation to the volume rendering integral, thanks to their separable ray-by-ray evaluation. Relatively simple implementations are also available for both Central Processing Unit (CPU) and Graphics Processing Unit (GPU) architectures. On the GPU, entry and exit points are commonly extracted from the volume bounding box in the rasterization step of the graphics pipeline.

A competing technique called texture slicing has also been proposed, but is today less common as it is arguably both more complicated to implement and less adaptable for non-standard scenarios or alternative architectures. For further details on implementation options or alternative solvers, the reader is directed to the work of Engel et al. [20]. 


\subsubsection{Sampling and Reconstruction}

So far in this section, data values have assumed to be available whenever and wherever needed. This is of course typically not the case, as data are mostly available as discrete samples on a grid. Whenever a sample point is invoked at an off-grid, its value must be computed from surrounding grid points. This process is called interpolation, or more generally, reconstruction. In Figure 2.1, reconstruction takes place in the 'filtering' stage of the DVR pipeline.

Modern DVR implementations on the GPU typically make use of the hardware supported tri-linear interpolation. Unfortunately, there are significant performance losses associated with anything else than tri-linear interpolation due to lack of hardware support. Popular alternatives to the tri-linear kernel includes both cubic and Gaussian variants. For later parts of the thesis, it should also be noted that despite spatial weights being the most common, sometimes additional weights are used. One example is the common bilateral kernel, which takes into account not only the distance between samples in the spatial domain (spatial distance), but also in attribute space (radiometric distance).

\subsection{Fused Data Visualization}

Earlier sections contained several examples of scenes with more than one data source. When data are neither the same size or have the same orientation, explicit methods are required in order to utilize the different sources within the same rendering. Where and when to access the different sources is thus an important consideration, as well as how to combine their respective contributions.

\subsubsection{Multi-volume Fusion}

When rendering multiple volumes, it is beneficial to know where to sample each individual volume. Scenes with two or more volumes are therefore divided into homogeneously occupied regions. This is illustrated in Figure 2.6 (top), where two objects result in three distinct regions: one region for either volume and one regions occupied by both volumes.

For DVR and raycasting, this introduces the concept of homogenous ray segments. Each viewing ray is split into consecutive segments as it passes through the homogeneously occupied regions, as illustrated in Figure 2.6 ((bottom)). Rendering parameters and sampling can, in this way, be optimized for different combinations of volumes.

In terms of the optical model, ray segments are naturally incorporated when the integral in Equation 2.2 (p. 13) is partitioned into the sum in Equation 2.3. 

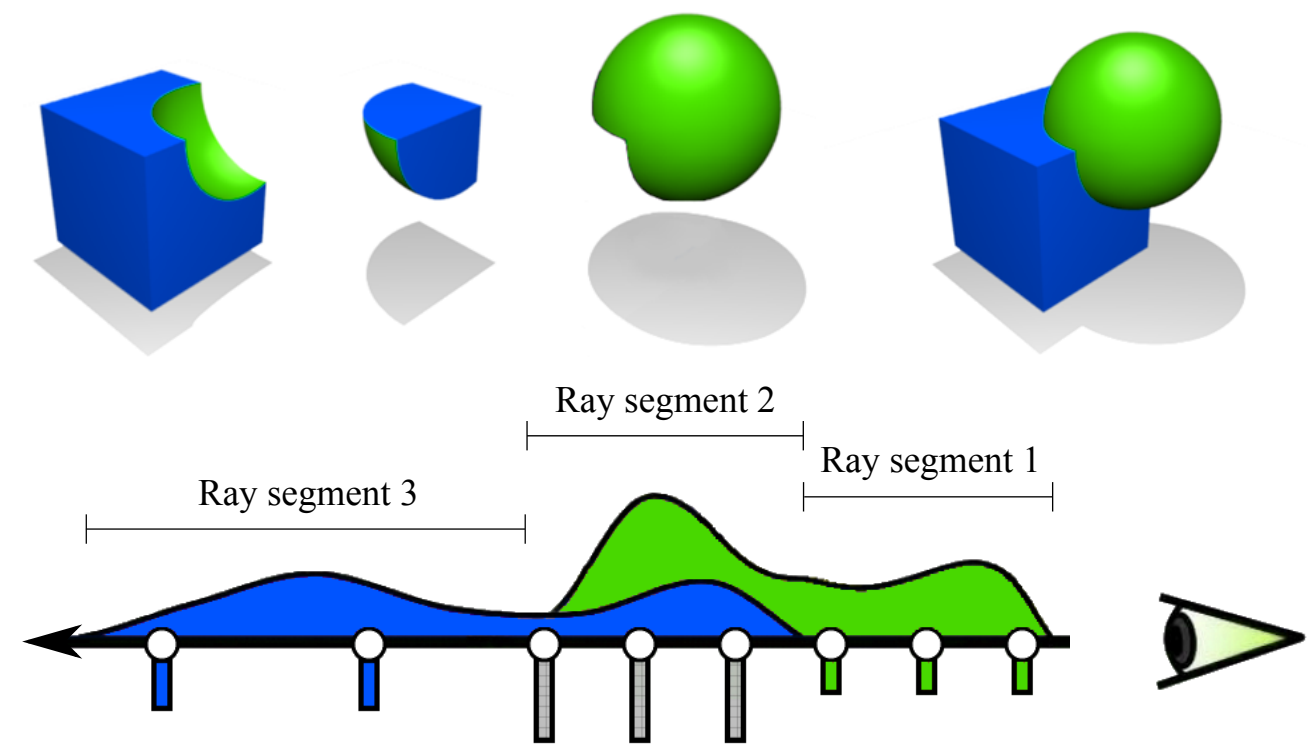

$\Delta$ Figure 2.6 - Scene partitioning by region occupancy (top) and the resulting ray segments (bottom). Original illustrations by Captain Sprite (top), licensed under CC-SA 3.0, and Engel et al. [19] (bottom). Colors were adjusted.

The ray would then be split into a set of homogeneously occupied segments before being partitioned into further segments for blending purposes.

The regions extracted for DVR purposes are nearly always convex, which greatly simplifies depth sorting for occlusion purposes. Convex regions also lend themselves well to space partitioning schemes. These algorithms have been used since the early days of computer graphics to speed up scene queries, by allowing large parts of the data to be ignored based on its spatial location. Common representations include Octrees, Kd-trees and Binary Space Partitioning (BSP), which all successively divide any space into smaller regions. They are naturally implemented as tree structures on the CPU.

Opaque geometry can be included in fusion scenes through an efficient two stage process [20]. A discussion on semi-transparent geometry, which requires very different techniques, is provided in Chapter 3.

\subsubsection{Levels of Intermixing}

Visual contributions from multiple sources can be fused at different stages of the DVR pipeline. Cai and Sakas [11] categorize three levels of intermixing in: illumination, accumulation and image level. Both the illumination and accumulation levels are performed for each step during raycasting, while the image 
level refers to the blending of two completed images. The illumination level also refers to the use of a single multi-volume illumination/TF model, while the accumulation level refers to the use of separate illumination/TF models for each volume. Unless otherwise stated, this thesis concerns accumulation level intermixing.

\subsection{Transfer Functions}

While the optical model of DVR relies on color contributions, most scientific data hold little information about optical properties of the depicted materials. Instead, a mapping is performed that converts observed data values into color and opacity contributions (cf. $c_{i}$ and $\kappa_{i}$ in Equation 2.2 p. 13). The function that controls this mapping is the TF.

One of the key roles of the TF in visualization is to provide a way for the user to interact with and explore the data. In a simple scenario, the user selects a range of data values that corresponds to a certain material, then selects the color for that material to be used in the rendering. The capability of the TF to separate different tissues, referred to as its specificity, is therefore a very important aspect when comparing different TF solutions. In general, the specificity of a TF depends on which, and how many, attributes (variables) are considered in the analysis. More attributes generally leads to higher specificity. The space spanned by the attributes is referred to simply as the attribute space, or alternatively, the TF space or classification space.

For rendering purposes, TFs are commonly stored as fixed resolution textures, known as look up tables (LUTs).

\subsubsection{Pre- and Post-classified DVR}

The TF is most often applied after the reconstruction step of the visualization pipeline in what is known as post-classified DVR. Sometimes the TF is first applied to all grid points before the resulting colors are interpolated for a final contribution. This is known as pre-classified DVR, but is less frequently used due to the overly blurry quality of the resulting images.

\subsubsection{One-dimensional Transfer Functions}

The arguably most common way to work with TFs is through saved presets with the option of manual adjustment through a dedicated editor. Figure 2.7 illustrates a standard one-dimensional (1D) TF editor, where the TF is overlaid on a background showing the histogram of the data. The horizontal axis 


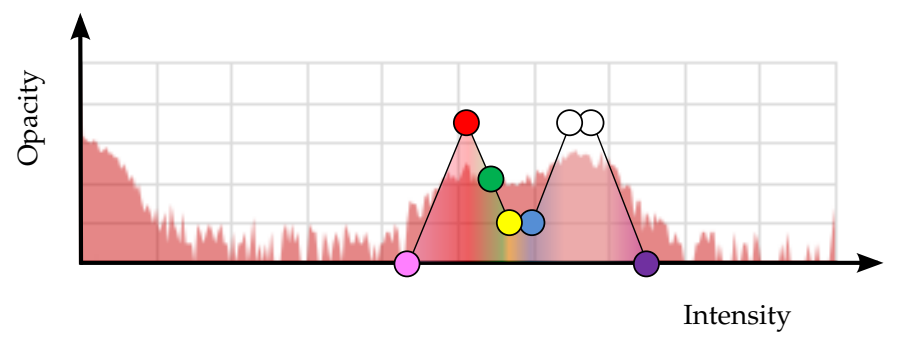

$\Delta$ Figure 2.7 - Piecewise linear TFs use control points for interaction.

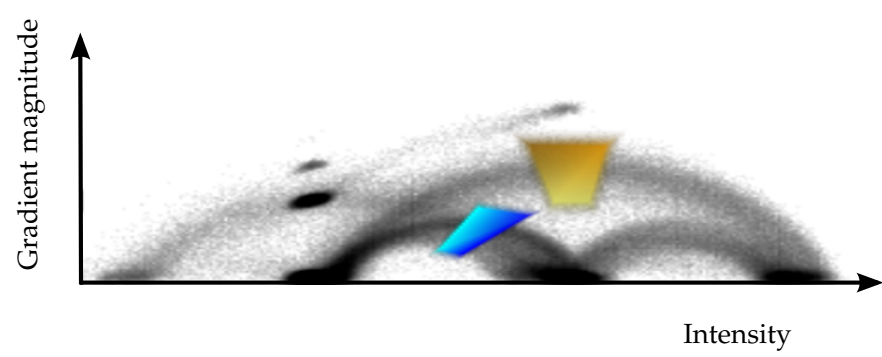

$\Delta$ Figure $2.8-2 \mathrm{D}$ TFs are used to increase specificity at the cost of complexity.

corresponds to the range of possible data values, while the vertical axis dictates the opacity of the output. In this example, the mapping from data values to visual contributions is defined through a set of, by the user, editable control points.

\subsubsection{Multi-dimensional Transfer Functions}

The TF concept is often extended to higher dimensions to improve the separability between different materials. Figure 2.8 exemplifies a 2D TF where the horizontal axis corresponds to the intensity of the data while the vertical axis corresponds to the magnitude of the gradient. Since the second axis has a dedicated attribute, opacity is typically edited only through visual cues. Any TF of more than one attribute is often referred to as a Multi-dimensional TF (MDTF).

A critical aspect of any MDTF solution is the increased interaction complexity that comes from the increased parameter space. This and related issues will be discussed in more detail in Section 3.4.

\subsubsection{Widget Based Transfer Functions}

In Figure 2.7, the TF is parameterized by control points. A different form of TF is shown in Figure 2.9, where the color mapping is defined through a set of 


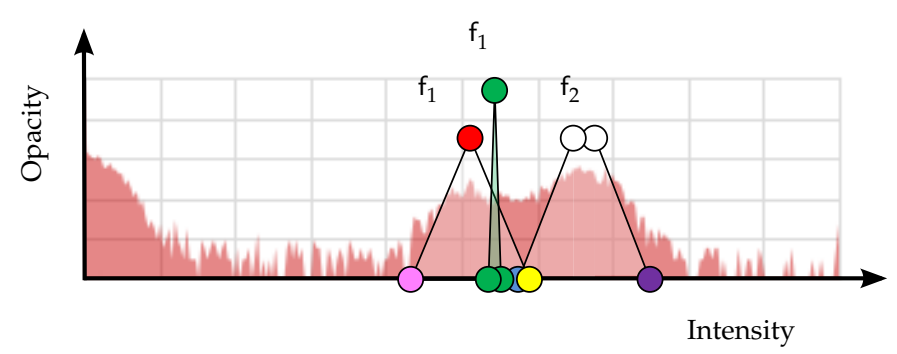

$\Delta$ Figure 2.9 - Widget based TFs are built from pre-defined primitives and can be extended to incorporate material labels (see $f_{1}$ and $f_{2}$ above).

widgets (graphical representations of primitives). Each widget is composed of multiple restricted control points, which tends to reduce overall complexity.

Individual widgets often correspond to particular features in the data, such as tissue types, opening the possibility to add semantic labels to the interface. Such labels will be used in later parts of this thesis to add additional widget-specific rendering conditions. Widget based TFs are the preferred solution in this work and a requirement for several of the concepts proposed in Chapter 4 . 



\section{3 \\ Trends and Challenges in Medical Volume Visualization}

In the last two decades, medical visualization and DVR has seen an immense development. Yet new challenges emerge as old problems are solved. This chapter will provide further detail into a few directions in which visualization research has diverged and underline some of its remaining challenges.

Before moving on to the technical challenges, however, the presentation begins with a motivation for why further research toward medical visualization is necessary.

\subsection{Challenges of Modern Healthcare}

Efficiency is already a major concern in medical imaging [2, 3, 74, 97]. At the same time, populations all over the world are increasing while people are living longer with less healthy lifestyles. As a result, the healthcare system needs to drastically increase its efficiency in the near future. To set this necessity for efficiency in perspective, consider the 'population pyramid' visualized in Figure 3.1.

The pyramid shows the number of people in various age groups for the population of western Europe. What can be read from it is that an increasingly large fraction of the population is middle aged or older. In terms of healthcare, this 


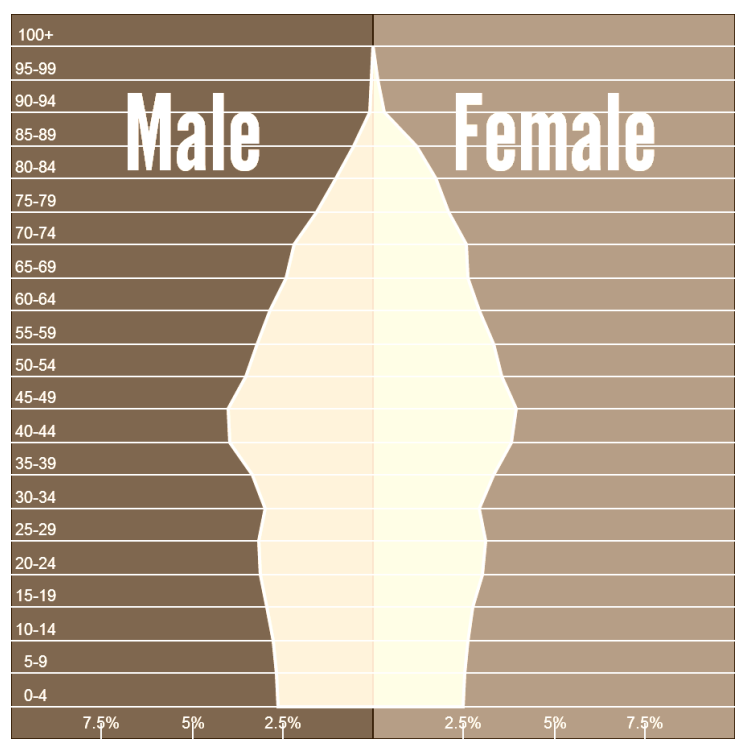

$\Delta$ Figure 3.1 - The population pyramid of western Europe shows an aging population with ever increasing healthcare needs. Visualization plays an important role in increasing the efficiency enough to address these issues. (c) populationpyramid.net. Reprinted with permission.

will require the system to increase its efficiency just to maintain the level of care available today.

At the same time, the amount of data generated for each patient and procedure is continuously rising, a trait often referred to as the 'data explosion'. One aspect of this data explosion is the increased size of the generated data, in terms of more images at higher resolutions. Another aspect is an increase in the number of different modalities that are used for a single procedure. Visualization already plays a key role in meeting these new challenges and its importance is only likely to increase.

Challenges, such as the increasing load on the healthcare system described above, have already shaped medical visualization and will likely continue to guide its growth in the future. Provided next is a brief timeline outlining a few key elements in the emergence of the visualization research community.

\subsection{Years of Visualization}

As a research field, volume visualization is relatively young. It is here outlined in three eras from the perspective of this thesis. 


\section{The Early Days}

In many ways, visualization first emerged as a subsidiary to the field of computer graphics. Early pioneers made their first contributions in the late 80's through works toward surface extraction algorithms and raycasting of volumetric data. The 90's brought refinements of the optical model as well as further development of the TFs concept in terms of design and classification. Multiple volume setups were also beginning to attract attention. New publication venues dedicated to visualization undoubtedly added to the growth of the field.

\section{The GPU Explosion}

DVR entered a new era around the turn of the millennium, triggered by the introduction of programmable GPUs. The new hardware proved to be a game changer which saw real-time interaction becoming a norm rather than an exception. Ensuing research investigated computational efficiency and performance trade-offs, including nominal work on pre-integrated volume rendering and explicit hardware techniques. The early 00's also brought further investigations into both translucency effects and extended lighting models for more photo-realistic renderings.

Many of today's sub fields were also being established at this time, including higher dimensional TFs, focus/context techniques as well as importance driven and labeled volume rendering to name a few. The concept of illustrative volume rendering also saw considerable growth along with other non-photo realistic techniques.

Comprehensive information on the state-of-the-art of volume rendering by the end of this era is well documented: see The Visualization Handbook by Johnson and Hansen [40], Real-Time Volume Graphics by Engel et al. [20], and Visualization in Medicine: Theory, Algorithms, and Applications by Preim and Bartz [92].

\section{The Modern Diversification}

Volume visualization has continued to broaden and has diversified in many more interesting directions than what is reasonable to list in this brief outline. Yet some general themes can be observed. Computationally expensive tasks, for example, have taken advantage of the ever increasing power of the GPUs with research related to even larger data, fusion of multiple data sources and more expensive forms of filtering and classification. At the same time, visualization has also been made more accessible to non-expert users through dedicated research on user interfaces and semi-automation of previously manual tasks. Visualization has also been further adapted towards public dissemination 
with further work on illustrative rendering schemes and exposure to new audiences through specialized hardware, such as visualization tables for public dissemination.

Yet perhaps the largest change lies in the shift towards new domains, including molecular and biological sciences as well as environmental data. Still, the medical domain remains one of the cornerstones of volume visualization, with increased focus on applied visualization and application aspects as core techniques have become more established.

The following sections will expand upon concepts of particular interest to this thesis.

\subsection{Advancements in Fused Data Visualization}

The first focus area is that of fused data visualization. Much like visualization at large, fusion has transitioned from the CPU over to the GPU, with recent work addressing the highly complex case of so-called hybrid data featuring both volumetric and geometric sources. Below, existing solutions from across this spectrum are presented along with their respective strengths and weaknesses.

\subsubsection{Geometry Based Approaches To Multi-volume Fusion}

A minimal fusion scenario may involve only a few volumes. Such scenarios have been addressed through intermediate CPU side containers called V-objects [28]. Blocked representations of the volume bounding boxes are used for increased entry/exit point granularity. Viewing rays are segmented into mono- and multi-volume clusters to improve cache performance. Other CPU side data structures have also been proposed, including pre-sorted texture slices [100], octree representations $[79,90]$ as well as BSP $[66,79]$.

In the last few years, focus has shifted away from the CPU. Even so, geometry based approaches are still valuable in many situations. Static scenes, for instance, require very little additional computation once a sufficient geometric scene partitioning is achieved. In contrast, GPU based approaches spend considerable resources recomputing much of the information for each successive rendering. Geometry based solutions are also widely applicable as they impose fewer requirements on the choice of rendering method and are more widely compatible with CPU side solutions such as the HDVR ${ }^{\circledR}$ renderer from Fovia, Inc. [23]. Additionally, space filling structures are also well suited for secondary tasks, such as containment queries. 
Challenge C.1: A challenge of geometry based fusion approaches is the maintenance and sorting of the geometric representations. Many previous works have utilized space filling structures which need to be combined with external methods for the necessary sorting. BSP presents a viable solution in this scenario. The use of the BSP structure was first proposed in Paper I and later combined with octrees to support larger volumes [79].

\subsubsection{Depth Peeling and the Transition to the GPU}

For highly dynamic or complex scenes, fusion has adopted a family of graphics techniques known as Order Independent Transparency (OIT). First out was depth peeling, which was initially introduced for fast rendering of semi-transparent geometry [81]. In depth peeling, successive depth layers are processed by iteratively rendering all scene geometry while extracting and processing only the nearest layer per iteration. The depth of the extracted layers are stored on a per-pixel basis between iterations to ensure that previously processed layers are ignored in subsequent passes. Multiple works have addressed the efficiency of the method, for example, by peeling multiple layers per iteration [4, 13, 21, 72].

When utilized in volumetric fusion, depth peeling solves the visibility sorting where each successive peel corresponds to a layer of ray segments. Depth peeling is commonly applied either directly to the volume bounding boxes [8, 101] or on specialized focus lenses over underlying octrees [90].

Challenge C.2: Despite extensive research efforts, depth peeling still requires the scene to be re-drawn multiple times, where the number of re-draws is dependent on the maximum depth complexity. This means that the efficiency of the approach decreases as the complexity of the data increases. In such cases, A-buffers have been shown to provide better performance $[44,124]$.

\subsubsection{A-buffers and the Inclusion of Geometry}

An increasingly common data source for fusion are geometric representations in the form of triangle meshes. As triangle meshes generally cannot be assumed convex, their inclusion in fusion scenes often result in drastically higher depth complexities. For example, a bundle of 30 fibers, each represented by a tube, could add anywhere between 2 to 60 depth layers even if no ray intersects the 

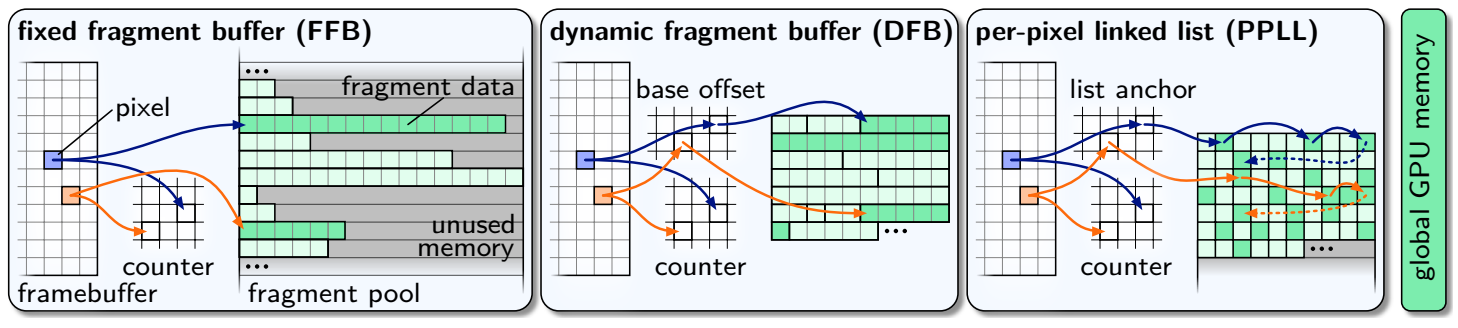

$\triangle$ Figure 3.2 - Three A-buffer implementations exist for managing global GPU memory. DFB and PPLL both adapt to scenes with non-uniform depth complexities. None of the methods include optimizations for local GPU cashes.

same tube twice. In contrast, a convex volume bounding box adds only two depth layers.

The A-buffer is an OIT solution designed to draw complex scenes in a fix number of rendering passes $[5,12,18,46,86,119]$. Only one or two passes are required depending on the implementation. In short, the A-buffer algorithm collects and sorts a list of intersection points per pixel, called a Fragment List, where the intersection points are computed from rasterized scene geometry. For fusion purposes, each pair of fragments in the Fragment List correspond to a ray segment.

The A-buffer algorithm performs two critical memory intensive tasks per pixel and frame: the construction of the Fragment List and its subsequent sorting. The first of these steps allocates and writes to global GPU memory, while the latter allocates and reads/writes to local GPU memory (cache memory or shared memory). Three approaches have been presented to optimize global GPU memory usage: Fixed Fragment Buffer (FFB) [15], Dynamic Fragment Buffer (DFB) [83, 118] and Per-Pixel Linked Lists (PPLL) [15, 42, 124]. These are illustrated side by side in Figure 3.2. A-buffers have been used for visualization of hybrid data [42].

Challenge C.3: While existing A-buffer techniques are very good at managing global GPU memory, they are less adapt at managing local GPU memory. The biggest challenge in the local case is the use of statically allocated arrays in the fast cache memory. As a result, existing algorithms enforce a trade-off between supported depth complexity and rendering speed. 


\subsection{Transfer Functions 2.0}

Aside from camera positioning and clip-planes, the TF is one of the primary interaction tools in medical volume visualization and thus an important focus area. Yet in its role as the link between the human and the computer, the TF is not entirely unproblematic. For one, the TF ultimately represents a trade-off. If classification specificity is maximized, interaction complexity usually suffers. If automatic solutions are applied, medical users do not trust the methods, and so on. These contradictions have instigated extensive research into evolving the TF in drastically different directions [89].

While the search for a panacea TF may be futile, advances in the field still increase the possibility to, for a given case, find some algorithm that strikes a reasonable compromise. This section presents some of the more established approaches.

\subsubsection{Classification Specificity and Multiple Dimensions}

A significant portion of the available research focuses on increasing TF classification specificity. Arguably, the most common way to achieve this is to increase the dimensionality of the TF to form an MDTF. Gradient magnitudes are perhaps the most straightforward extension, which are used to improve identification of boundaries between materials [62]. Further derivatives can be added to form boundary emphasis functions targeting material transitions [48]. These methods also form the basis of the first widget-based MDTFs [50,51], allowing users to manually create 'thick' isosurface like representations.

Several works explore local statistical measures or variations of the derivative approach. Examples include local intensity structures such as sheets and blobs [106], statistical moments [113, 114] and moment curves [88], as well as estimations of local mean and standard deviations [33, 102]. Local histogram correlation [75, 76] and low-high (LH) histograms [107] are also proposed. Additional secondary attributes include feature size [14] and shape [91, 106]. The core principles of the above mentioned works are very similar in finding a particular space or metric which makes the targeted materials separable. Once such a space or metric is found, unique materials can be identified based on their unique projection or 'footprint'.

Challenge C.4: There are a number of problematic consequences of increasing TF dimensionality. The primary of these are probably the interaction costs associated with the drastic increase of the parameter space. As more 
parameters get exposed to the user, difficulties involved in designing a TF grow drastically and the process becomes increasingly ad-hoc. Increased parameter spaces also make optimization strategies more complex and computationally demanding. Another, less frequently mentioned consequence, is the storage requirements of LUTs. For 2D TFs based on control points this is less problematic. But for higher dimensional MDTFs, or widget based 2D TFs where each widget needs to be accessed separately, storage increases drastically. Parametric and analytically evaluated TFs have been proposed [52] and are also used in many of the works of this thesis. Even so, maintaining a lower dimensional interface is still key to maximize applicability in the medical domain.

\subsubsection{Explicit Spatial Information}

One branch of TF research focuses on the connection between the classified features and their spatial location. This is of particular interest for the medical field, as the spatial location of tissues can be important in the diagnostic process. The related research can broadly be divided into two categories based on the source of the spatial information: methods that rely on topology and methods that rely on segmentation.

One benefit of including topological information is that tissues in different parts of the body can be distinguished even if they cannot be separated by the TF. Visualizations can also be partly automated in terms of color selection. Methods in this category typically rely on tree structures, where the data are divided into subsequently smaller nodes [25, 110, 111, 121, 122]. For example, if the data is a human, the highest node might be the body, which is then split into upper and lower parts. The upper body can then be split into head, torso and arms, and so on. Critical points, such as joints or branches, are often found by studying the behavior of isosurfaces in the scalar field.

Segmentation is used for medical visualization purposes, such as pro-operative and surgical scenarios [37, 98, 108]. The algorithms themselves are many and varied and detailed descriptions would be outside of the scope of this thesis. What is more relevant here are their application within TF design. In one approach, individual visualization settings are created for all materials and then applied only within each material's own spatial extent as defined by the segmentation [31,34]. Linking the visual output of the TF with the distance to the nearest segment border has also been proposed [112], as well as to extend the area-of-effect of the TF to outside of the segmented regions [45]. Segmented data have also been used to select focus regions in importance driven rendering, 
which effectively modulates the output of the TF to ensure visibility of selected features [120].

Challenge C.5: In specific cases, the inherent feature focus and quantitative measures can outweigh the cost of segmentation. Unfortunately, there remain many cases where segmentation simply does not work or where its performance is insufficient. Furthermore, potential imperfections in the segmentations or uncertainties in the data may critically endanger user trust, something which is particularly important in the medical domain.

\subsubsection{High-level Semantics and Work Flow}

Closely related to the quest for increased specificity is the strive for simple and intuitive interfaces. Several works focus on lowering interaction related costs by having the user sequentially interact with lower dimensional projections [14, 32, $76,91,125]$. Another way to aid the user is to supply additional information to aid the design process. A frequent form of guidance for TF creation is the utilization of the global data histogram as a background in the editor. This allows the user to identify peaks and place widgets accordingly. In an extension of this idea, local histograms are used to expose smaller peaks that are not easily detected in the global histogram [75, 76]. Semi-automatic methods that rely on segmentation or feature space analysis to identify unique tissues are also utilized $[39,80]$. Another extension builds on presets provided by recurring settings as motifs [109]. Higher-level user interfaces can be used to couple semantic expressions to parameters in the TF. Approaches include both principal component analysis of user input [99] and text based user input [94, 96]. A natural form of interaction, which is explored, is to 'paint' the TF directly on the data $[29,30,117]$ with a work flow that is reminiscent of the Photoshop imaging software. The aim of these methods is to facilitate a more natural or efficient creation processes, preferably using language from the user's own domain. This aim is also shared by many of the papers included in this thesis.

Challenge C.6: One of the biggest challenges related to semantics and work flow is how to make a simple interface without restricting the user. Again, the contradictory nature of the TF means that there is no universal solution to this challenge, but many situationally acceptable compromises. One way to increase compatibility of different TF extensions is to focus on solutions that do not extend the classification space. 


\subsection{Visualization of Vessels}

Vessels have long been one of the primary applications for volumetric visualization due to their inherent 3D structure. Many sophisticated methods have been proposed to find, analyze and visualize vessels. In a survey by Preim and Oeltze [93], these methods are broadly divided into model-based and modelfree surface methods as well as methods that rely on DVR and TFs. A key benefit of TF centered approaches over surface algorithms is a lower dependency on expensive skeletonization or segmentation techniques.

\subsubsection{Vesselness Measures and Transfer Functions}

Vessels can be identified and located much like any other feature using a TF. 1D TFs are, however, rarely sufficient for this task, which is why higher-dimensional or computational approaches are often used. A particular family of filters used in this scenario are simply referred to as vesselness filters. Vesselness is often analyzed through locally applied filters designed to identify tubular structures through the Hessian matrix [24]. Sato et al. [106] propose a multi-scale extension to this filter in order to identify vessels of varying thickness. Similar filters based on polar neighborhood intensity profiles have also been proposed [41]; reportedly achieving better results in non-tubular sections.

TFs for vessel visualization can be constructed in many ways. Image centric approaches include stochastic optimizations as well as approaches based on multiple renderings [35, 82, 123]. Vesselness measures can also be directly integrated in the TF. The vesselness can then be exposed to the user through the use of an MDTF [106] or remain a hidden system parameter that automatically modulates vessel opacities [41].

Challenge C.7: There are several challenges related to TF driven vessel visualization. Particular challenges of MDTFs have already been discussed in C.4, p. 27. Vesselness filters, and how they are best used in combination with a TF, are also problematic. Using them directly to modulate opacity carries a risk of over-emphasizing non-vessel structures [58]. Such a solution is also further complicated due to the often varying intensity in different regions of the vessel.

\subsubsection{Centerlines and Segmentation}

Segmentation based vessel analysis relates to methods such as centerlines, curved planar reformatting and surface representations. The literature on this 
topic is extensive, but well categorized by Lesage et al. [61] and summarized by Läthén [57]. Automatic parameter adjustments provide an excellent example of how segmentation data can be used in visualization [26].

While centerline and segmentation based approaches are valuable in many scenarios, they are often based on interaction schemes or have parameter spaces which make them less suited for the same clinical work flow as TF driven approaches. Therefore, only a brief summary is provided below.

The categorization provided by Lesage et al. [61] separates works that rely on photometric, geometric and feature driven models. The previously mentioned vesselness filters are here categorized among the feature driven models. Extraction methods are subsequently categorized into region growing algorithms, active contours and centerlines. Many of these algorithms rely on seed points or representations of internal and external forces. For particular contributions the reader is referred to existing surveys [10, 49, 61, 92].

\subsection{Discontinuities and Partial Volume}

Visualization often depicts data where the object of interest consists of discrete features. Our understanding of the human body, for example, includes discontinuities at multiple levels. At a larger scale, features such as major organs are seen as separate entities. On smaller scales, tissues or cell types are also considered in distinct classes. For visualizations of phenomena with discontinuities to be intuitive, it is important that the discrete nature of the features is preserved.

Unfortunately, today's imaging modalities do not deliver even a fraction of the resolution necessary to delineate discontinuities at all scales. As illustrated in Figure 3.3, at just below $1 \mathrm{~mm}$, today's x-rays cannot identify single cells but are able to identify larger features. To make things worse, most image acquisition techniques will result in convoluted data due to frequency limiting filtering operations (anti-aliasing). One of the effects this has on the data is referred to as partial volume, i.e. that many smaller volume parts, originating from different tissues, contribute to a single voxel value.

Challenge C.8: Partial volume is an ill-posed problem that can only be solved in full for synthetic data. Methods devoted to this problem are thereby forced to rely on assumptions and approximations to find the boundaries or to come up with alternative representations of the uncertainty. It is also not straightforward how data values near a boundary should be treated, even if the location of the boundary can be estimated. In medical 


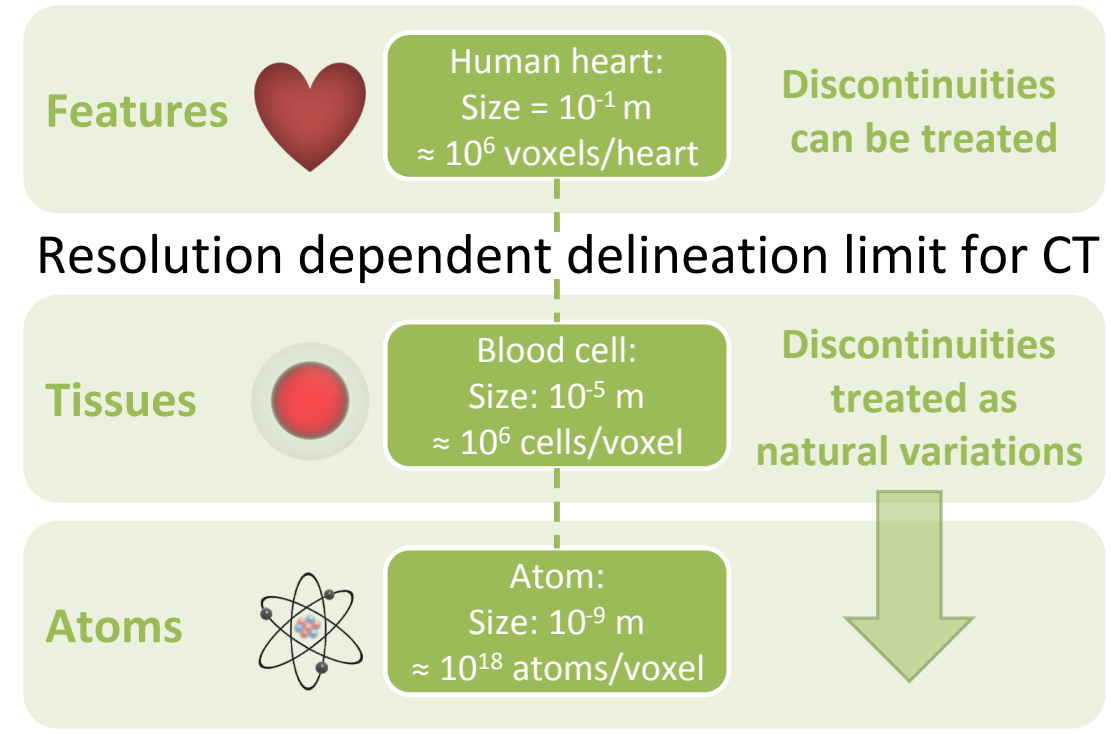

$\Delta$ Figure 3.3 - Discontinuities exist on many scales but can only be represented for larger scales in visualizations due to the limited data resolutions.

visualization, partial volume has the undesirable effect that voxels may be assigned intensity values unrelated to any of the originally participating tissues. For example, a voxel with partial volume contributions from both air and bone may be assigned an intensity value identical to the heart muscle.

The literature devoted to partial volume and its effects is extensive and only a brief review is within the scope o this thesis. Below, selected approaches are divided into three categories: visual classification, explicit segmentation and statistical models. Regardless of the category, the output is typically one of the following: either the signal is reconstructed as fully continuous and eventual misclassification is dealt with in the subsequent classification step, or the signal is reconstructed as a piece-wise constant function of discrete material classes.

\subsubsection{Visual Classification}

Without continuous data interpolation, such as in the case of pre-classified DVR, partial volume often creates a speckled noisy pattern in feature transitions. In this case, a continuously interpolated representation, and its associated 'sheet' artifacts, may instead be preferable. To remove the artifacts altogether, the visual approach relies on the user to construct a sufficiently good TF, primarily through higher dimensional variants. Gradient based MDTFs aimed at isosurface 
like representations of material transitions were introduced in Section 3.4.1. The same methods can also be applied to address the effects of partial volume, as the transitional regions can be either classified explicitly or avoided in the TF design $[48,51,62]$.

Challenge C.9: A problem with the MDTF approach is that it, by design, is better at creating visual representations for the transitional regions than it is to suppress their associated artifacts. For example, creating an MDTF widget for even a single solid material is not a straightforward process. To do so, extensive manual manipulation is required to include all transitional arcs related to the targeted material in the design. This is not only time consuming, but also causes further misclassification at low gradient magnitudes where the arcs inevitably start to overlap.

\subsubsection{Explicit Segmentation}

Another way to address discontinuities in visualization is through labeled volume data. To be fair, these methods do not identify material transitions themselves as much as they rely on these transitions to be provided through complimentary label volumes containing per-voxel material designations. Segmentation based methods also tend to focus mostly on smooth binary representations [31, 115] without representing the uncertainty of the transitions. Even so, segmentation based approaches can be used to reduce classification artifacts by spatially constraining the TF response near uncertain regions. Any binary aspects could potentially be addressed by the use of weighted distance fields [45].

Challenge C.10: The challenges of segmentation have already been discussed in C.5, p. 29, with existing methods being specialized or reliant on time consuming manual interaction $[1,85]$. There are still many cases for which reliable, unsupervised segmentation procedures simply do not exist. Methods that rely on labeled data will, in these cases, remain situational.

\subsubsection{Statistical Models}

Statistical models can be been used to reverse engineer the partial volume effect, either into percentile tissue components or into probabilistic expressions [36, 56]. Probabilistic classifiers and statistical tools, such as maximum likelihood 


\section{Chapter 3 - Trends and Challenges}

estimation, are then used to output either multiple values (weighted estimates) or a single value (the most likely estimate) per data point. The former case retains more information related to classification uncertainty, which has been used in numerous works [53, 55, 77, 103]. For an overview of statistical models and their application in image processing, the reader is referred to the works of Santago and Gage [104] and Gonzalez and Woods [27].

Challenge C.11: A common trait for the statistical models used in visualization is that they rely on complete data models, i.e. that all materials in the data are known. While this makes sense for data consisting of relatively few tissue types, it has a number of inconvenient consequences in the general case. First, all data point will be forced to belong to the available classes, even if they in reality belong to a material not included in the model. Second, all potentially occurring materials need to be modeled even if the visualization only concerns a single material, resulting in increased costs related to both computational load and interaction complexity. 




\section{4}

\section{Medical Volume Visualization Beyond Single Voxel Values (contributions)}

This chapter describes and contextualizes the contributions from the papers included in this thesis. The presentation follows the theme of combining and utilizing information sources beyond single voxel values, from improved methods for data fusion to efficient user driven visualizations.

\subsection{Overview of Contributions}

The contributions of the individual papers are illustrated in Figure 4.1 in relation to the DVR pipeline. Paper I \& II both relate to data fusion, while Paper III-VII address interaction efficiency and visualization effectiveness. More specifically, Paper III-V consider secondary information from filters and external data while Paper VI \& VII rely on user provided classification. With the exception of the evaluation in Paper IV, all contributions can be used as standalone components or be combined into a larger pipeline.

A shared aspect of many of the presented approaches is the goal to emphasize important data to achieve more effective visualizations. To this end, the visualization pipeline traditionally includes a 'data filtering' or 'pre-processing' stage related to the exclusion of less important data. Techniques such as focus and 


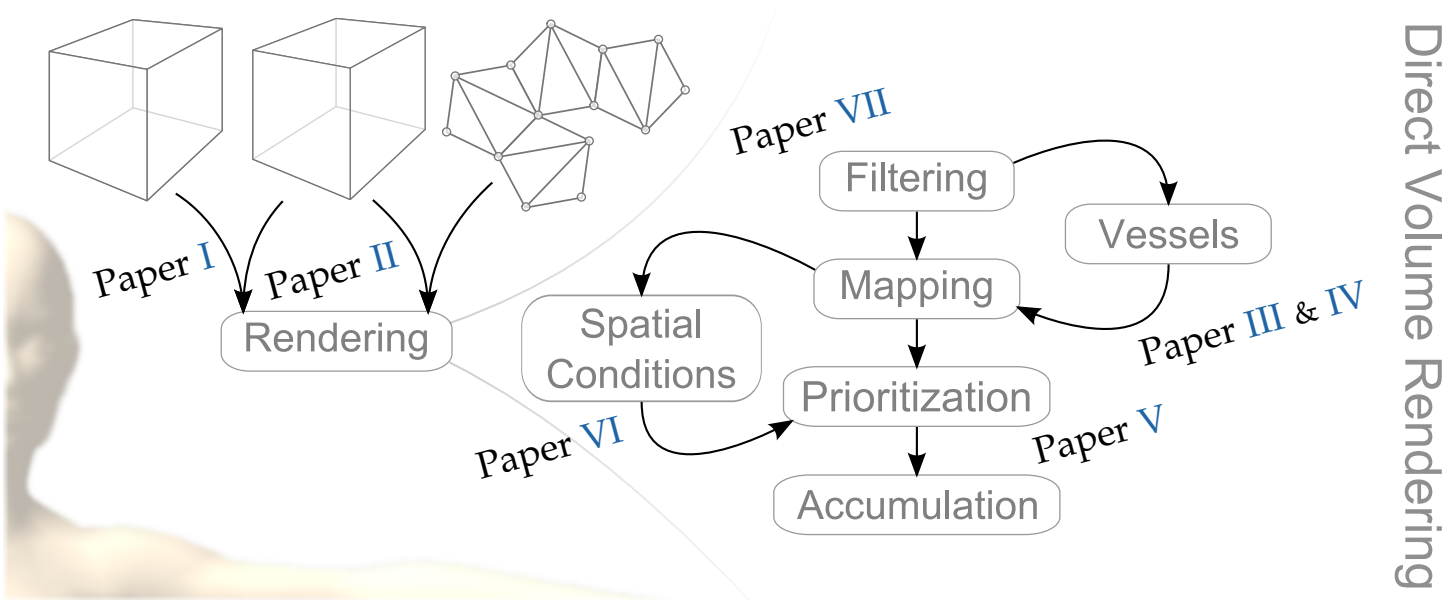

$\triangle$ Figure 4.1 - Illustration of the contributions of this thesis in relation to the DVR pipeline. Original illustration theme provided by $\AA$. Birkeland [6].

context rendering suggest, however, that much can be gained by de-emphasizing rather than removing contextual data. This is here referred to as data prioritization and introduced as an explicit stage in the DVR pipeline in Figure 4.1. As indicated by its position towards the end of the pipeline, it is separated from the mapping stage and is applied on the mapped samples candidates.

\subsection{Consolidation of Multiple Data Sources}

In order to utilize information from more than one source, the information must first be aligned and consolidated. This section presents two methods to the field of fused data visualization targeting different stages of the rendering pipeline. Both methods address performance penalties associated with the enforcements of painter's algorithm.

\subsubsection{Binary Space Partitioning for Volumetric Fusion}

The first contribution to fused data visualization primarily targets scenes containing a limited number of volumetric objects. The approach uses BSP to produce a region based scene description. It is designed to use a single data structure to provide both the scene description as well as the necessary sorting, which was identified as a deficiency of existing approaches in C.1, p. 24. The use of BSP also provides flexibility regarding the choice of rendering algorithm and maintains a low overhead during camera interaction. The approach was originally presented in Paper I. 

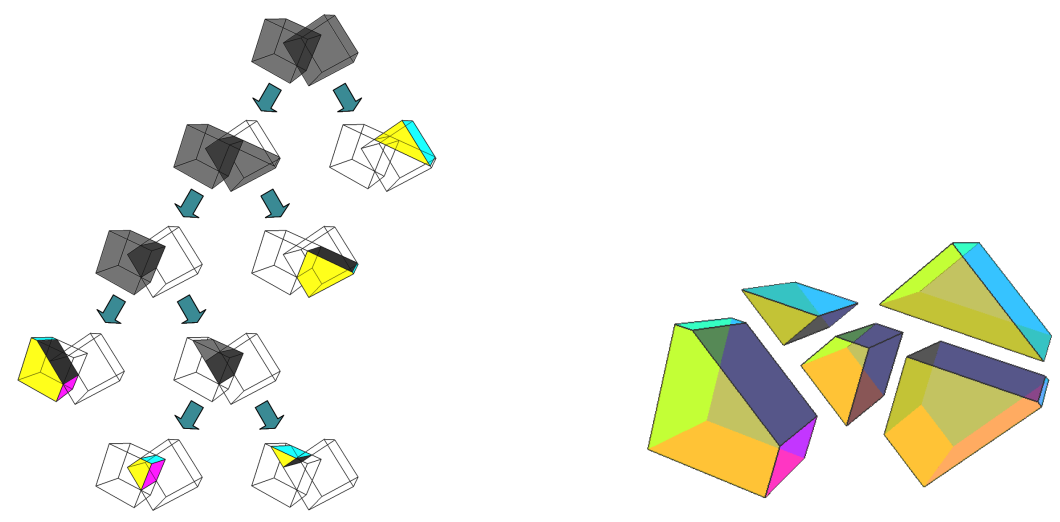

$\triangle$ Figure 4.2 - BSP subdivision of two overlapping volumes. Images show the BSP-tree (left) and the resulting convex regions (right).

\section{Plane Selection and Space Partitioning}

The use of BSP for the purpose of tracking volume occupancy results in a cost efficient scene description with a set of homogeneously occupied, convex regions. BSP is a non-axis aligned algorithm well-suited to produce accurate representations for intersections of multiple convex polyhedra [87]. Its output is a binary tree structure, referred to as the BSP-tree, where the top node represents the entire space and the leafs represent the smallest homogenous regions.

BSP has a number of benefits of particular value in a fusion scenario: the bounding boxes of the participating volumes provide excellent starting polyhedra and guarantee that all nodes and leafs are convex, the planes of the initial bounding boxes guarantee sufficient partitioning without further input, the plane normals provide efficient sorting of nodes and leafs in depth relative any point in space, and the partitioning planes can additionally be used for efficient inclusion queries.

Figure 4.2 illustrates the subdivision process for a scene containing two volumes. The construction of the BSP-tree (left) results in a set of convex regions (right). (Note that the volumes are aligned in one of the three dimensions to simplify the example.)

The proposed algorithm operates directly on the polyhedra bounding boxes of the participating volumes and the criteria for terminating the subdivision is that no polyhedra boundaries are left in the interior of any regions. The only exception to this rule are single polyhedron regions, which can be terminated without further subdivision. 


\section{Performance Considerations}

The performance of a BSP process heavily depends on scene complexity. Two scenes with the same number of volumes may have drastically different complexity, in terms of unique homogenous regions, due to volume placement. In Paper I, two scenes were constructed with five volumes each: one with with well below 200 and the other with ca. 400 volume fragments. BSP generation time for the two scenes were timed at $2.25 \mathrm{~ms}$ and $9 \mathrm{~ms}$. Due to the recursive nature of the generation, the use of object pools and paged memory allocation is recommended. Performance improvements by a factor of ten were observed for access and allocation times using the above optimizations.

\subsubsection{Per-Pixel Depth Complexity Adjustments for Hybrid Data}

The second contribution to fused data visualization primarily targets dynamic scenes with hybrid data consisting of both geometric and volumetric objects. Significant performance improvements are achieved for A-buffer based algorithms to overcome the performance related challenges of OIT algorithms outlined in C.2 \& C.3, pp. 25-26. The method exploits the characteristically non-uniform depth complexity distributions often found in scenes with hybrid data. Paper II presents two components, Per-Pixel Array Optimization (ppAO) and Per-Pixel Depth Peeling (ppDP), designed to improve performance by adapting the A-buffer pipeline to non-uniform depth complexities.

\section{Non-uniform Depth Complexities}

In Paper II, depth complexity distributions for rendered images are analyzed through Depth Complexity Histograms (DCHs). In short, a DCH portrays the distributions of depth complexities for a specific scene, camera and viewport. A DCH is computed by first generating a complexity image where each pixel holds its associated number of fragments produced by a full scene rasterization. A standard histogram over the complexity image then forms the $\mathrm{DCH}$, such that each bin holds the number of pixels that fall within a certain complexity range.

Figure 4.3 shows a hybrid scene consisting of the human protein carbonic anhydrase II (left). The scene's complexity distribution is illustrated in the intermediate depth complexity image (center) and the resulting DCH (right). Through the $\mathrm{DCH}$, it is possible to quantify the amount of pixels for a certain minimum complexity. In the protein scene, for instance, less than $10 \%$ of the pixels have a depth complexity of 16 or higher. (Note that the DCH is plotted in log-scale.) 

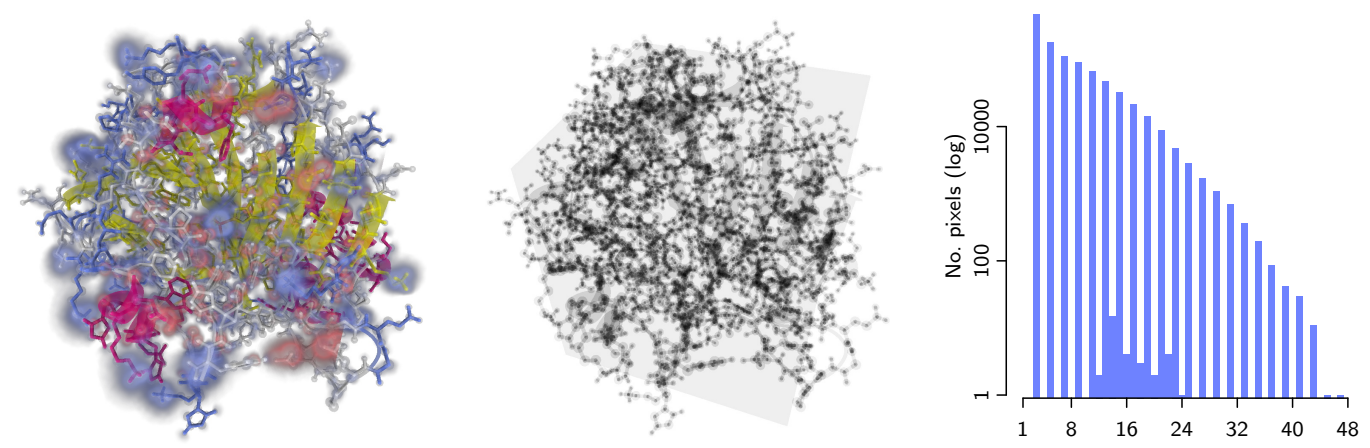

$\Delta$ Figure 4.3 - Hybrid data scenes (left) often have non-uniformly distributed depth complexities (center), resulting in rapidly decreasing DCHs (right).

A-buffer implementations commonly avoid expensive read/write operations on global GPU memory by first copying the data to local cache memory. Due to limitations in dynamic allocation, existing methods select a globally appropriate cache array size to be used for all pixels. For the protein scene in Figure 4.3, the ideal value would be $N=64$, which is also the most frequently reported number among existing literature. Knowing that $90 \%$ of all pixels have a depth complexity of 15 or less, the fixed array size results in a lot of wasted memory. The ppAO and ppDP components are designed to reduce this waste.

\section{Semi-dynamic Resource Management and Overflow Prevention}

The purpose of the ppAO component is to trigger each pixel with an as-small-aspossible array size. A set of pre-defined array sizes (generally powers-of-two) are defined and instantiated in separate shader programs. Image pixels are then split into segments of similar depth complexity before the rendering of each segment is triggered with the smallest available array size.

The integration of ppAO into existing A-buffer implementations is simplified in that the segmentation step can be performed implicitly as a masking step using a stencil buffer or equivalent. Integration is also greatly facilitated by the fact that most A-buffer implementations already produce the necessary per-pixel depth complexities as part of the core pipeline.

Pixels of very high complexity can still become a bottleneck, or even be impossible to evaluate, if large enough arrays cannot be allocated. The ppDP component addresses this issue by splitting each array into a sequential series of segments of size $M$. Using insertion sort and a 'current minimum depth' threshold, the algorithm finds, sorts and resolves the front-most $M$ fragments (that clears the threshold) per iteration. This way, much smaller array sizes can be used for 


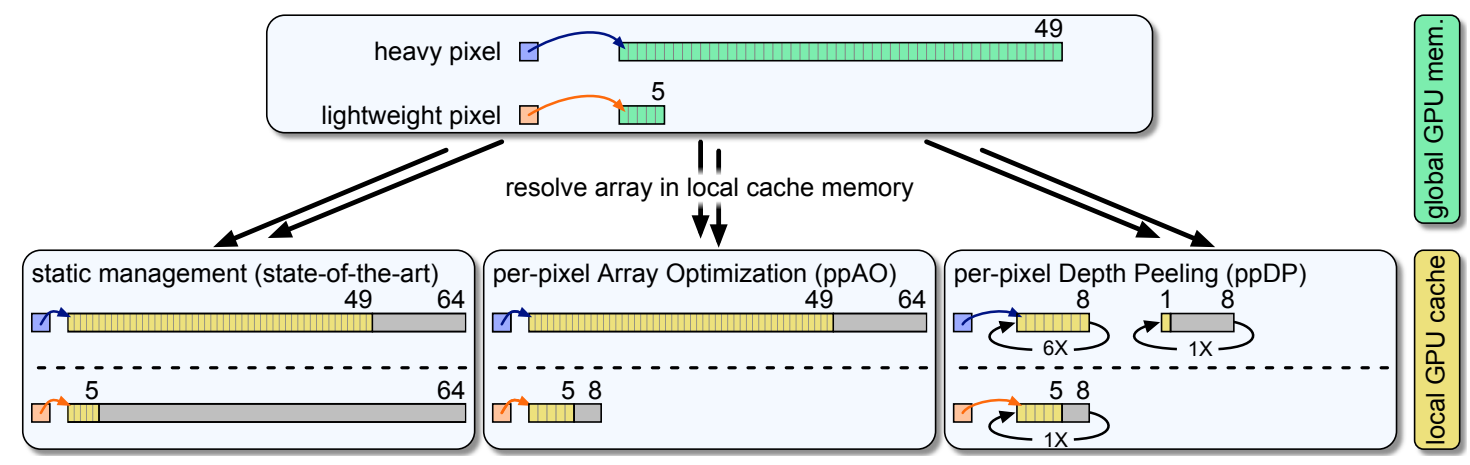

Figure 4.4 - Illustrations of the ppAO and ppDP components for A-buffer optimization in relation to static cache allocation.

highly complex pixels. ppDP also prevents the maximum size of the local array from limiting system complexity support.

Similar to standard depth peeling, the ppDP approach is susceptible to z-fighting issues. The issues could potentially be resolved using additional flags to separate processed from non-processed fragments, at the cost of additional read/write operations. Unlike regular depth peeling, the ppDP component operates fully on the GPU with no need to redraw scene geometry.

Both the ppAO and ppDP components are illustrated in Figure 4.4, where they are compared against static cache allocation.

\section{View dependency and Applicability}

Figure 4.5 shows the resulting frame time with and without the proposed components (red and blue line plots). The background shows the complexity distribution as screen coverage for different complexity segments with the DCH of every tenth frame stacked vertically. As can be seen in the performance graphs, significant gains are achieved regardless of scene configuration and system load.

The two components can be combined or executed separately. Both should also be compatible with most A-buffer implementations and therefore have wide applicability in both visualization and computer graphics.

The possibility to apply A-buffer techniques to highly complex data sets is shown in 4.6, which is rendered at $12 \mathrm{fps}$ using the proposed techniques. Static allocation yields $2.5 \mathrm{fps}$ in the same setup, but then also discards all but the first 64 layers. 


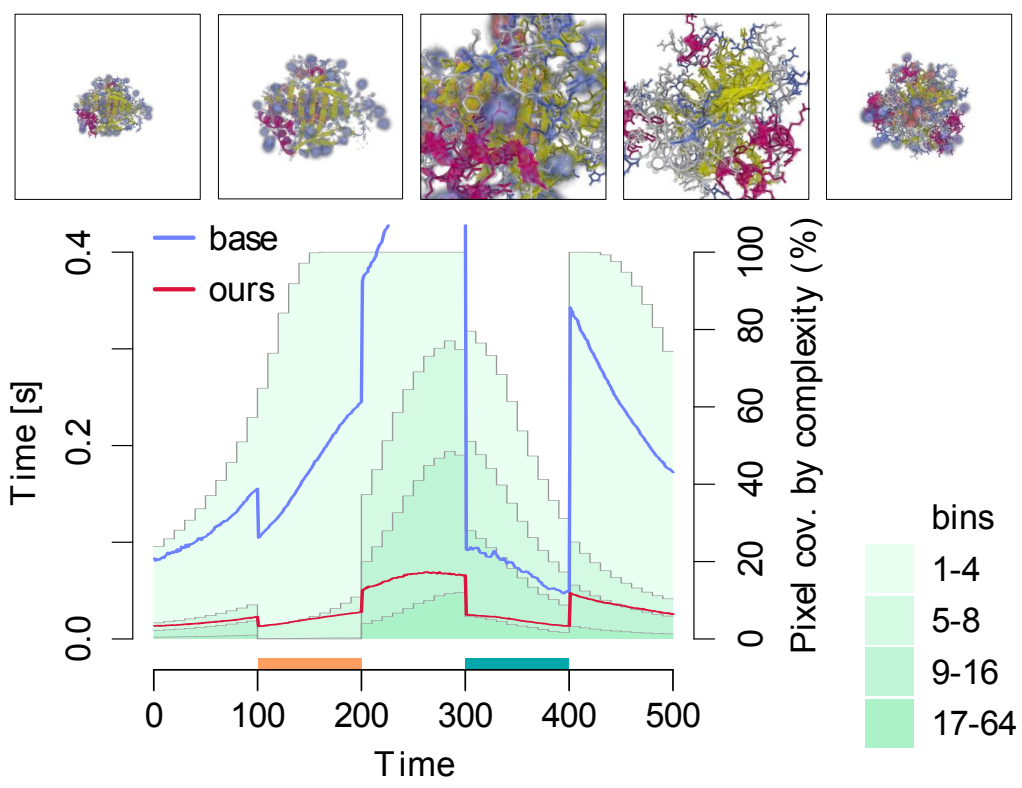

$\Delta$ Figure 4.5 - The plot shows rendering performance during camera animation. The scene starts out the same as in Figure 4.3, but changes in composition every 100th time step. Rendering times are shown as lines (left y-axis) and DCHs as bar charts (right y-axis).
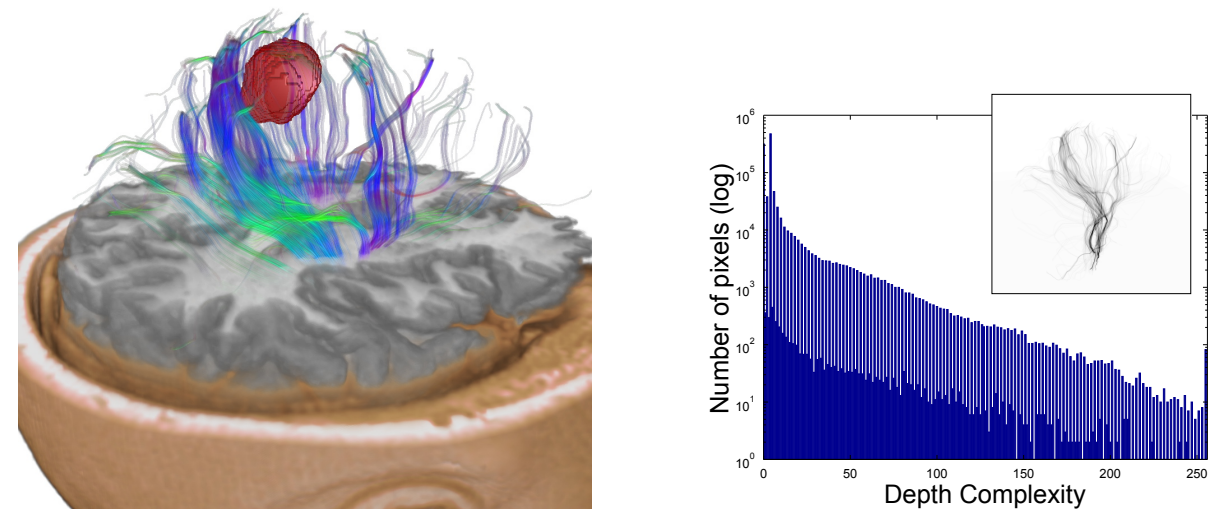

$\Delta$ Figure 4.6 - Visualization of hybrid data in the form of volumetric data of a human head and geometric representations of neuro pathways near a tumor (left). The pathways drastically increase depth complexity, but only for certain areas of the rendered image, resulting in a rapidly decreasing DCH (right). 


\subsection{Feature Prediction and Data Prioritization}

As noted in Chapter 1, a major challenge of volumetric visualization is to find and highlight only the data the user wants to see. This section will present two solutions targeting different aspects of this problem. One is a technique which emphasize vascular trees by automatically adjusting the TF to local vessel variations. The other is a technique for efficient anatomically related region-ofinterest (ROI) selection, with a particular focus on requirements for their clinical deployment.

\subsubsection{Automatic Transfer Function Tuning Based on Vesselness}

Visualization of blood vessels is a good example of how assumptions regarding the targeted features can be used to identify and retain important information. For vessels, these assumptions relate to their cylindrical nature and the vesselness filter discussed in Section 3.5. As detailed in C.5 \& C.7, pp. 29-30, however, challenges remain in regards to how best to utilize the output of a vesselness filter in a user driven visualization.

A particularly challenging aspect of CTA examinations is that contrast agents are rarely distributed uniformly throughout the body. This leads to variations in captured image intensity, with differences in the observed vessels as a result. Complete views of the vascular tree are therefore hard to achieve, and TF settings need to be changed when focus shifts from one region to the next.

These challenges are addressed in Paper III through an algorithm capable of automatically adjusting the TF for local intensity changes. A qualitative study of the method is provided in Paper IV. Both works are included in the dissertation of Läthén [57].

\section{Computational Vesselness Measurement}

When adjusting TFs for viewing CTA data, clinicians follow a procedure similar to an optimization process. Optimal TF settings are found by continuously adjusting the TF widget along the intensity axis until the best possible representation of the vessel is achieved on the screen.

The automatic algorithm presented here follows the same principles as human clinicians. Instead of observing the image, however, the method instead relies on vesselness filters applied in object space to the TF mapped data. For each grid point, the TF shift that produces the highest vesselness score is selected and stored as the optimum local shift. During rendering, the local shifts are automatically applied as the TF is sampled. 


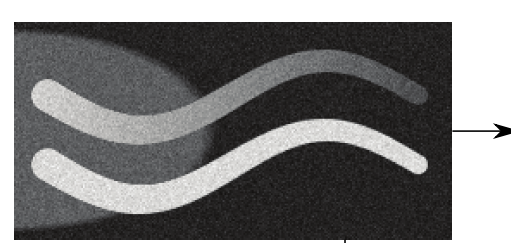

(a)

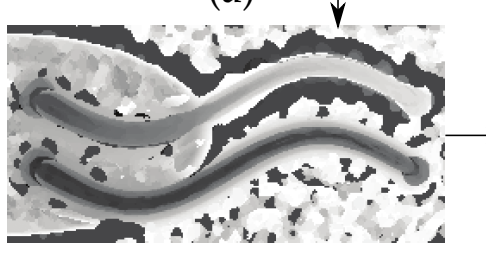

(c)

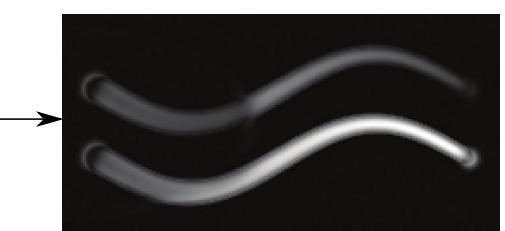

(b)

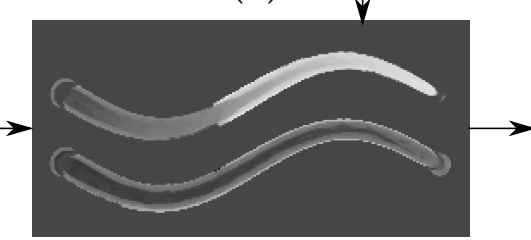

(d)

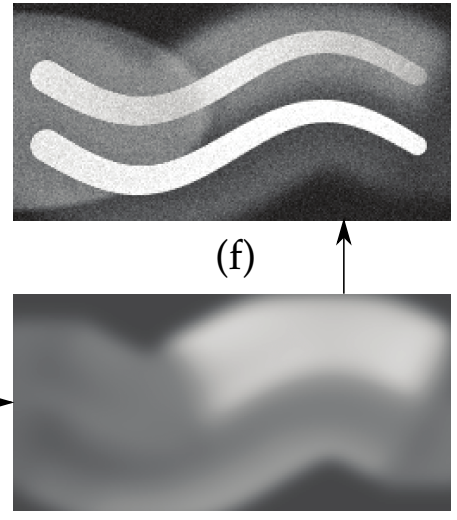

(e)

$\triangle$ Figure 4.7 - Overview of local TF shift parameter computation using a synthetic data set and a simple 1:1 grayscale TF. The data (a) is first first analyzed in raw form by a multi-scale filter (b) and in TF mapped form by a single-scale filter (c). The filter outputs are combined to a localized shift image (d) which is regularized (e). Applying the shift parameter to the TF yields highlighted vessels in the image output (f).

The computational measure of vesselness used in this work was originally proposed by Sato et al. [105]. In addition to the original multi-scale filter, our work also use a single scale variant. The size of the single scale filter is chosen to be larger than the largest vessel in the data. This makes the filter response dependent on vessel size, with increased magnitudes for thicker vessels. Maximizing this measure brings out the largest vessel-like structures of the data.

\section{Regularization}

Once the initial single-scale filter response is computed, the algorithm is finalized by a regularization. The response is first morphologically extended through a weighted averaging operation, before being smoothed with a Gaussian filter. This regularization serves to prevent the TF from changing too rapidly between neighboring voxels. The filter output is also weighted by the output of the original multi-scale filter. The purpose of this weighting is to prevent the TF from being manipulated in regions where no vessels exist. The computation of the shift parameter and its regularization is illustrated on a synthetic data set in Figure 4.7. 

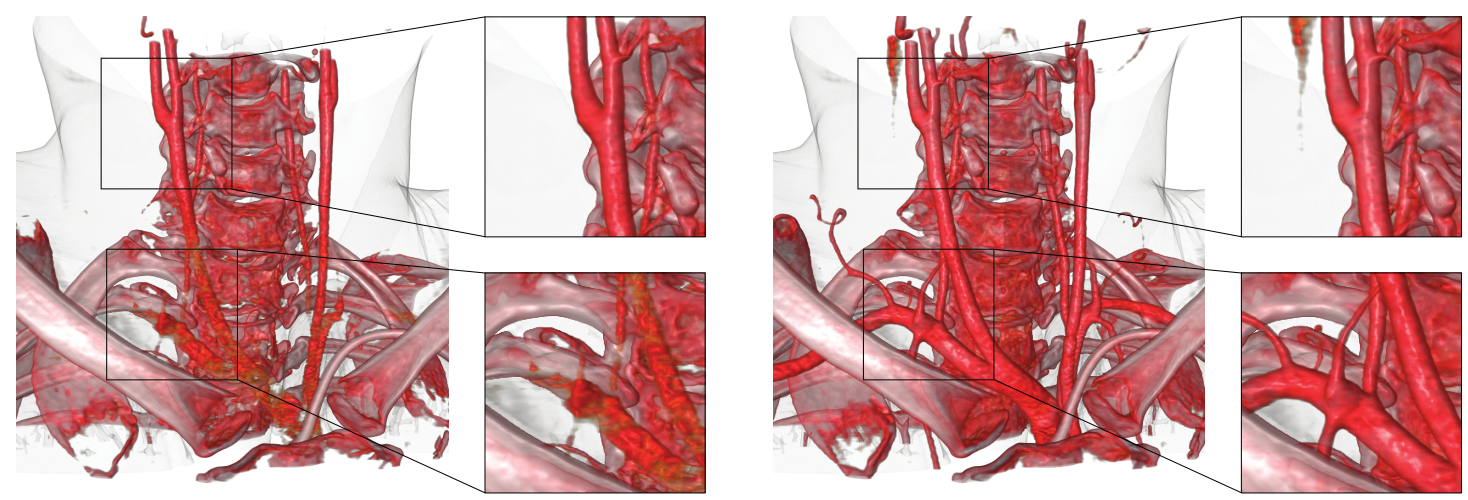

$\Delta$ Figure 4.8 - Visualizations of complete vascular trees are difficult due to variations in contrast agent concentration in CTA. The images show a standard preset TF (left) and the proposed method (right), with magnifications on two interesting regions along the patient's right carotid artery.

\section{Results}

The results of the method are demonstrated in Figure 4.8, which shows a CTA examination of the carotid artery. Visualizations for a standard preset TF (left) and the proposed method (right) are both shown, including magnifications of interesting regions. The case exemplifies how the proposed method only marginally alters the visualization in regions where the preset is sufficient (top magnification), while regions with poor preset output are properly adjusted (bottom magnification).

The clinical feasibility of the method was investigated through a user study in Paper IV. The primary focus of the study was to gain understanding of the limitations and benefits associated with this form of vessel visualization. Therefore, a qualitative approach was selected. In short, the study found that standard TFs often lead to overestimations of the vessel lumen and that locally adjusted TFs can help to alleviate this problem. This is exemplified in Figure 4.9, where the three images show the slice data (left), global TFs (center) and locally adjusted TFs (right). The participants did find that the interpretation of the locally adaptive method to be less intuitive, but also noted that this did not introduce any prohibitive complexity in the work flow.

\subsubsection{Registration Based Regions-of-Interest}

Many features or generic ROIs in the body cannot be found through filtering in the same way as the vessels discussed above. In such cases, other forms of data prioritization is necessary to avoid the problems of globally applied TFs, illustrated previously in Chapter 1 Figure 1.3, p. 3. 

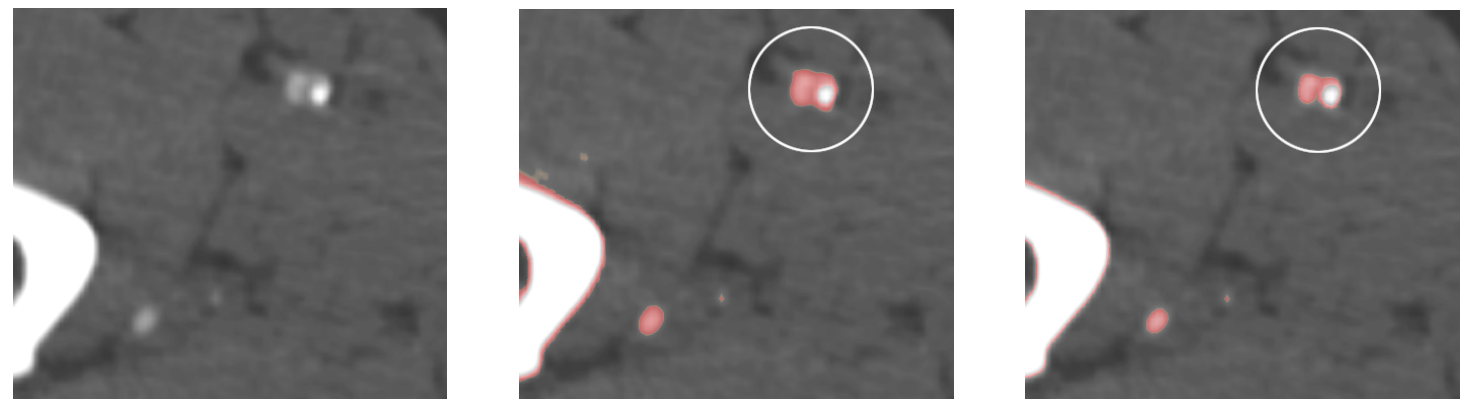

$\Delta$ Figure 4.9 - The study in Paper IV found that standard TFs sometimes lead to overestimation of the vessel lumen, a problem that was less pronounced with locally adjusted TFs. The images show the slice data (left), standard TFs (center) and locally adjusted TFs (right).

Anatomical data prioritization has traditionally been based on accurate label volumes or tree structures (cf. Section 3.4.2). Unfortunately, as noted in C.4 \& C.5, pp. 27-29, neither label volumes or tree structures are readily available or easy to construct. Presented below is a registration based approach for automatic anatomical ROI (AA-ROI) designed to alleviate these limitations. The approach was originally presented in Paper V.

\section{Localized Rendering Based on Atlas Compartments}

Distance based TFs $[45,112]$ represent an interesting niche of research well suited for data prioritization. The concept is straightforward; once the position of a targeted feature is known, the surrounding data can be emphasized or de-emphasized based on its distance to the targeted feature.

Two ways of de-emphasizing data are considered in this work: opacity reduction (which modulates sample transparency) and saturation reduction (which modulates sample color). De-emphasizing data can of course be done in many ways, such as illustrative methods [9,95] or alternative shading schemes [31, 34]. Another interesting option is to use sparsity instead of transparency to achieve a similar effect [47]. The interface used in the work of this thesis relies on label association instead of text based interfaces, as proposed by Rautek et al. [94]. The same form of de-emphasizement and interface is also used in Paper VI, Section 4.4.1.

Using registration to power the AA-ROIs increases efficiency, since the same generic atlas is used for all patients. Deformable image registration on the GPU is performed automatically in under 30 seconds. This registration is often performed between the patient data and model data, rather than directly with the atlas itself. Patient aligned atlases are acquired by applying the displacement 

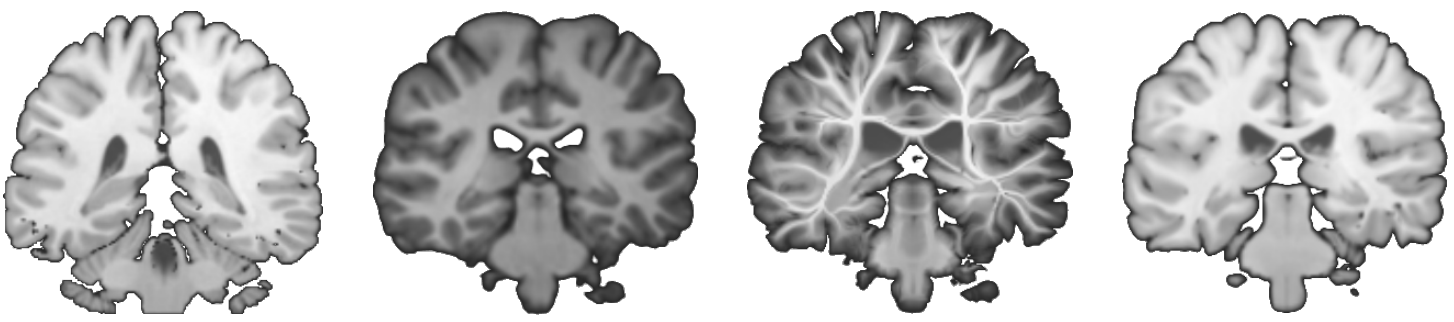

$\Delta$ Figure 4.10 - Choosing the right registration algorithm is important. Leftto-right: labeled atlas data before alignment, patient data with vastly different intensity ranges, failed alignment with intensity dependent Demons algorithm and successful alignment with the intensity independent Morphons algorithm.

field from the registration onto the generic atlas. Figure 4.10 highlights the need to consider data characteristics when choosing registration algorithm.

\section{Requirements for Clinical Deployment}

The work in Paper $\mathrm{V}$ focuses on general requirements for successful clinical deployment of AA-ROIs. A set of prerequisites were first set up in collaboration with clinical professionals before a prototype software was implemented. Important factors for clinical deployment were then investigated through a user study; the full results of which are available in Paper V. In short, the clinicians participating in the study judged that the concept of AA-ROIs could increase interaction efficiency. AA-ROIs would be particularly useful for tasks that presently require extensive use of clip-planes, such as the case shown in Figure 4.11, where the visualizations depict the internal structures of a pair of human lungs.

The availability of atlas data and registration accuracy are arguably the most critical factors determining the applicability of the approach to specific cases.

\subsection{Probabilistic Classifiers and User Knowledge}

The domain knowledge provided by the user is a highly valuable but challenging source of information. Medical professionals, for instance, rely on large amounts of experience in their diagnostic work. The proficiency of human users in patterns recognition tasks is also well known. At the same time, any human computer interaction needs to be fluid and intuitive.

The focus of this section is on methods based on user input. In particular, a TFlike interface is used to construct probabilistic material classification functions, hereafter denoted classifiers. Two options are explored: either the classifiers are 

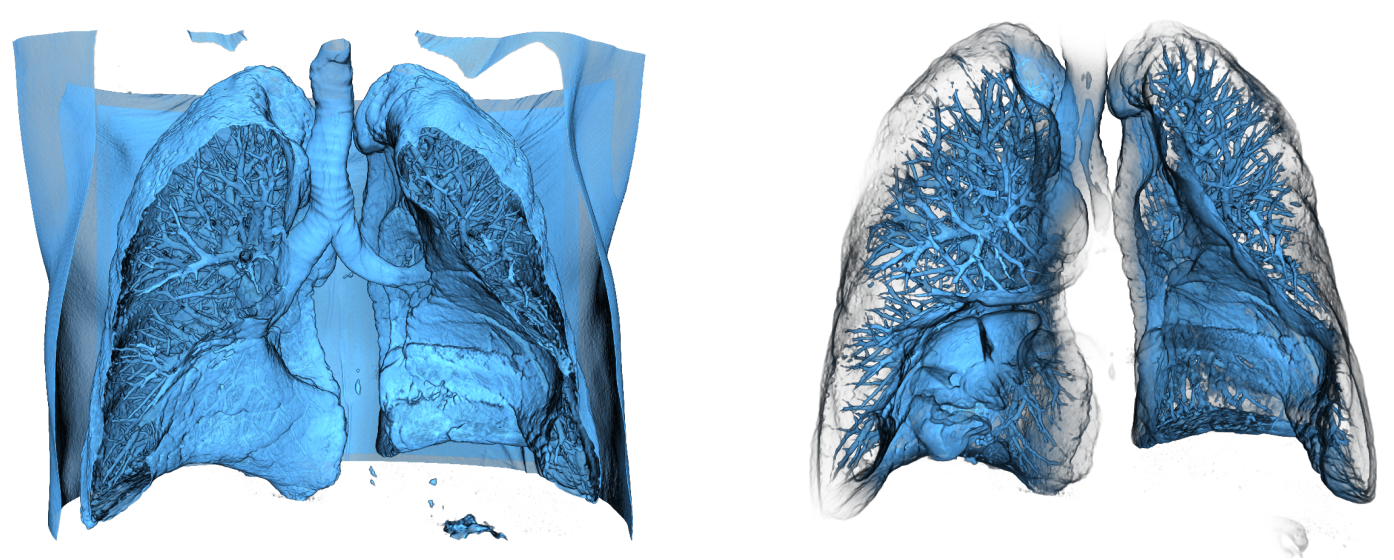

$\triangle$ Figure 4.11 - Clip-planes are not always sufficient to expose internal structures (left). AA-ROI selection can help reduce the necessary interaction (right).

derived from the opacity channels of the TF primitives, or the classifiers are constructed using separate widgets (typically in a separate editor). Both options are illustrated in Figure 4.12 and result in a set of initial user estimations

$$
P\left(C_{m} \mid y\right): \text { user supplied probabilities, } m \in[1, M] \text {. }
$$

These user estimations are then normalized to ensure that $\sum_{m=1}^{M} P\left(C_{m} \mid y\right) \leq 1$. In Paper VII the normalization is designed to preserve the relative magnitudes of the individual estimations. An extended discussion on normalization schemes is available in Section 5.1. The normalized estimations are then complimented by a null class, $C_{0}$, such that $m \in[0, M]$, before being used in probabilistic models

$$
P\left(C_{0} \mid y\right)=1.0-\sum_{m=1}^{M} P\left(C_{m} \mid y\right) .
$$

The role of the null class is to handle the 'remaining', unidentified classes not explicitly expressed by the user. The null class also ensures that the combined probabilities sum to one without the need for potentially strong normalization. Classifiers of this form are used in both Paper VI \& VII to address the challenges of data prioritization and boundary representation. (Note that the classifiers are referred to as 'material presence functions' in the original manuscript of Paper VI.)

\subsubsection{Clinical Data Prioritization by Spatial Conditioning}

Spatial relations between tissues have a prominent role in image-based radiology. For example, in the diagnostic procedure, a radiology examination of a patient 

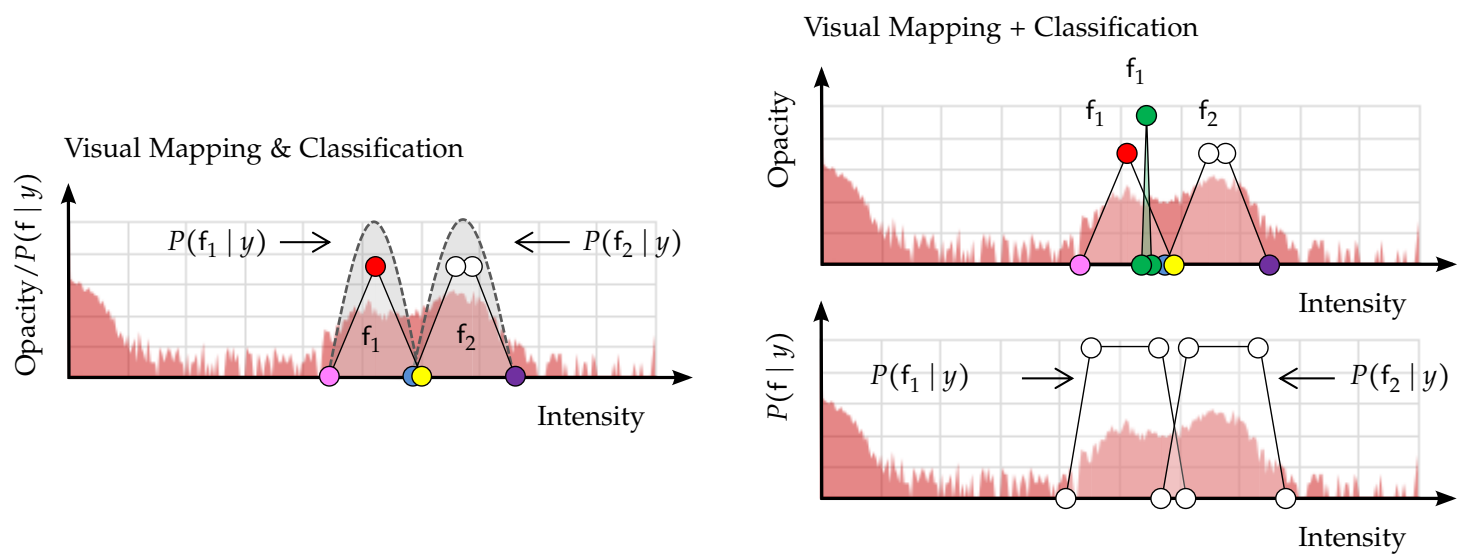

$\Delta$ Figure 4.12 - Feature classifiers are either extracted from the visual primitives (left) or edited as separate widgets (right).

is often based on hypotheses expressed by more than one tissue. The expression "metastases in the liver", for instance, refers only to a specific subset of all possible metastases.

Including spatial relations in the DVR approach creates a very effective form of data prioritization. The approach shares many similarities to the atlas-based data prioritization described earlier, but relies on a very different form of feature delineation. This delineation comes from the use of feature classifiers, which are used to identify and encode material footprints in the attribute domain. The approach is related to the overall goal of data prioritization, as well as the interface/workflow related challenges listed in C.6, p. 29. It was originally proposed in Paper VI.

\section{Forming Material Dependencies}

Figure 4.13 exemplify spatial conditioning through three different visualizations, all targeting internal air pockets. The images show: a naïve visualization which includes all air in the data set (left), a visualization of a thin slab (center) and a visualization with the spatial condition to "only render air when close to blood" (right). (The slab visualization is presently the prominent way to expose the inner air pockets.)

The user would create the conditioned visualization, Figure 4.13 (right), by selecting the corresponding widget in the TF and adding expressions based on the semantic labels of the classifiers. To enforce the condition, the method first analyzes the data to find regions containing blood. The result of this analysis is then encoded and stored as Neighborhood Meta-Data (NMD). During rendering, the TF widget associated with air is first evaluated to acquire a 

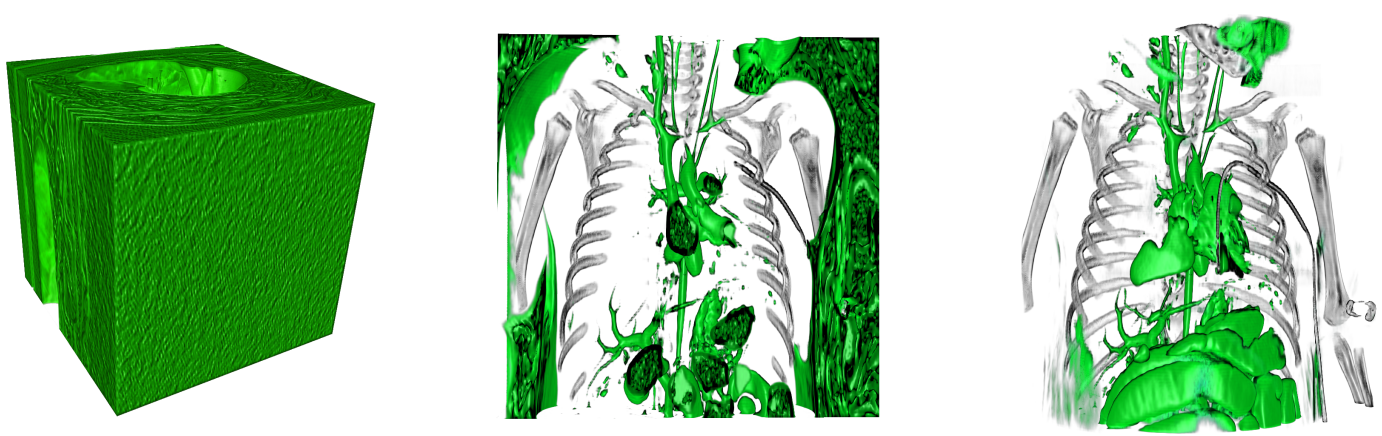

$\triangle$ Figure 4.13 - Standard TFs cause all air in the data to be equally emphasized (left). Viewing of internal air pockets becomes a time-consuming slab-by-slab procedure (center). The spatial condition "render air if close to blood" effectively emphasize only clinically important air pockets (right).

candidate visual contribution. This contribution is then conditioned according to the amount of blood that is present at the current sample position, as given by the previously encoded NMD. This way, the TF is prevented from creating any visual appearance of air in regions that do not also have a presence of blood. A conceptual method overview is provided in Figure 4.14.

\section{Material Footprints in the Attribute Domain}

In Paper VI, the classifiers are referred to as 'material presence functions', named so after their purpose of estimating and encoding spatial material distributions as scalar NMD. The encoding of the NMD is performed by averaging the classifier response in local neighborhoods of the data using a normalized box filter. A simple approach in assigning neighborhoods is to subdivide the data into cubic blocks. Half overlapping blocks are recommended for increasing the smoothness of the resulting NMD data, i.e. twice as many blocks per dimension as in the simple approach. Using unique neighborhoods for each original voxel was found not to be worth the computational cost.

The amount of data is reduced in two ways relative to approaches storing local histograms. First, each neighborhood is only represented by as many scalar values as there are classifiers. Second, the process effectively reduces the dimensionality of the data prior to storage, compared to directly storing the local histograms. The second form of data reduction is key in enabling the approach to also be applicable for higher-dimensional data. For example, storing the NMD at a resolution of $256^{3}$ and 8 bits will consume $16 \mathrm{MB}$ of memory. Storing local histograms of $16^{2}$ samples at the same granularity would consume 4.2 GB. 


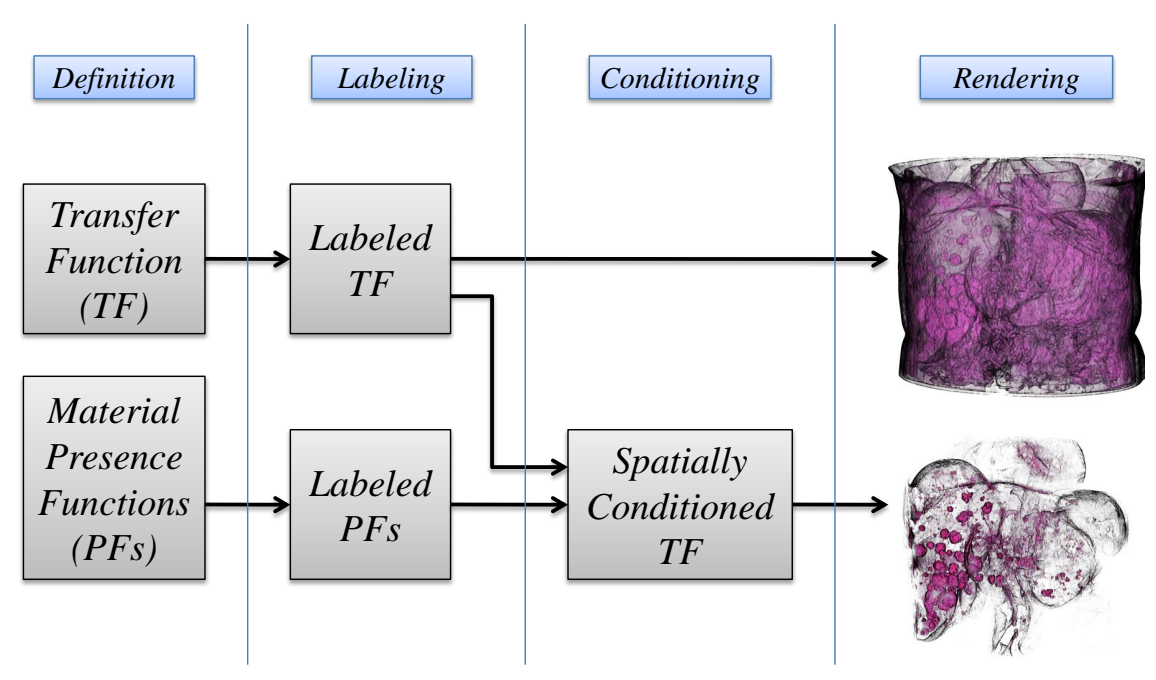

$\triangle$ Figure 4.14 - Material relations is an effective form of data prioritization. Semantic conditions, such as "render iodine when close to liver", allows the user to express important relations in his or her natural language.

Appropriate neighborhood sizes for the NMD depend on its intended use and the physical size of a voxel. Common sizes range between $4^{3}$ and $16^{3}$ voxels, with smaller features naturally requiring finer NMD granularity. Note that the granularity does smooth the data, as it only effects how fast the TF is conditioned in the spatial domain.

\section{Method Applicability and Accessibility}

The work presented in Paper VI addresses two explicit needs in medical volume visualization: the ability to express spatial relations between tissues and extensibility to higher dimensional data. The method is purposefully designed to require minimal changes to existing DVR pipelines in order to promote applicability. The use of high-level semantics is also designed to make the method directly accessible to non-technical users. Figure 4.15 shows the applied algorithm including two ways to de-emphasize less prioritized data.

\subsubsection{Improved Representations of Discontinuities}

The primary way to deal with discontinuities today is to analyze a local region of the data and to select a most likely tissue out of all possible tissues. Section 3.6 discussed some of the issue with this approach, see C.9, C.10 \& C.11, pp. 33-34. The work presented below follows a different concept in addressing the identified challenges. Instead of considering all possible tissues, the approach only 

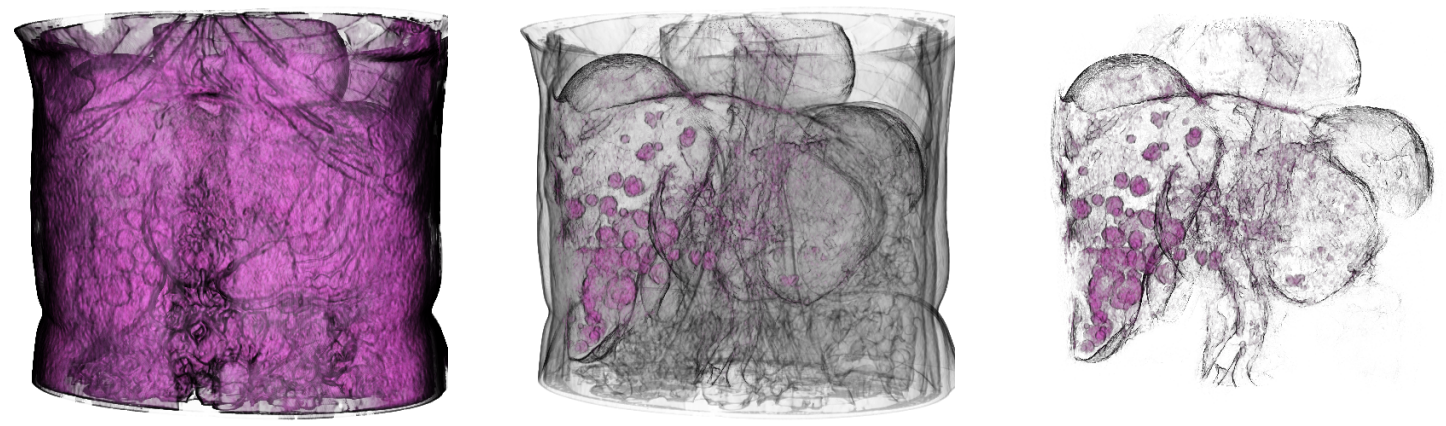

$\Delta$ Figure 4.15 - Visualizations of "iodine in liver": standard DVR (left) versus data prioritization through desaturation (center) and opacity modulation (right).

considers features that are actually visualized, i.e. features with currently active TF widgets. At every sample point the method also not only estimates the probability for each visualized feature, but also estimates what signal value each feature would have.

The value estimates is performed thorugh a feature-specific reconstruction stage, which is what separates the approach from related literature. Reconstructions are here weighted to prevent undesirable interpolations, such as the 'air + bone $=$ heart' problem described in C.8, p. 31. The classifiers discussed earlier in this section are used for feature weights and visual contributions from all active features are combined to produce a final visual representation.

The result is a novel piece-wise continuous data representation with non-sharp transitions. The approach was first presented in Paper VII.

\section{A Piece-wise Continuous Data Representation}

Extraction of feature specific signals from the data is performed by weighting the neighboring grid values differently for each targeted feature. The process is illustrated in Figure 4.16. For reference, a non-weighted reconstruction of the signal estimate $\hat{z}(x)$ would take the following form

$$
\hat{z}(x)=\frac{\sum_{x_{i} \in \Omega} w_{i} y_{i}}{\sum_{x_{i} \in \Omega} w_{i}}
$$

where $y_{i}$ and $w_{i}$ are the data value and spatial weight for grid point $i$, and $\Omega$ is the local neighborhood of all samples with non-zero spatial weights. To make 


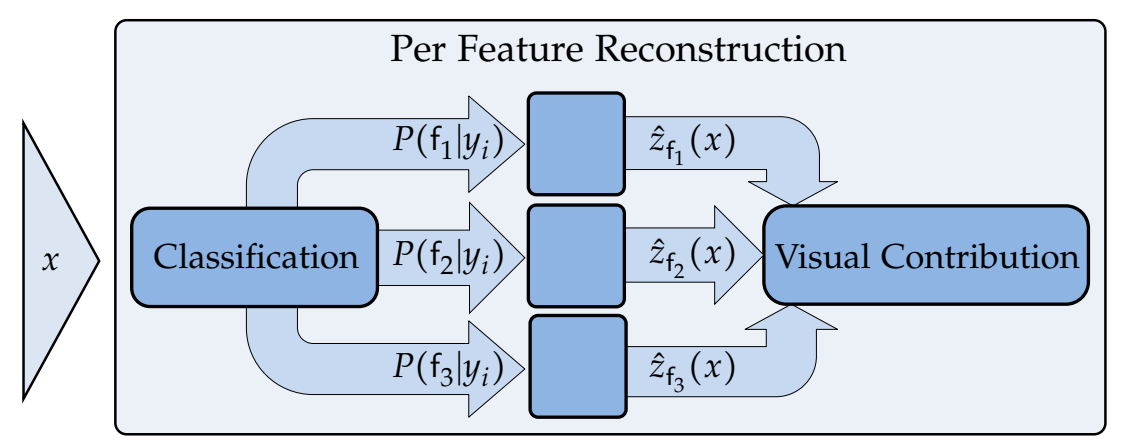

$\Delta$ Figure 4.16 - Uncertainty in boundary areas often lead to misclassification artifacts. This is addressed by performing multiple feature-weighted reconstructions which are combined to a single output.

the reconstruction specific for material $f$, an additional weight term is added to each grid sample

$$
\hat{z}_{\mathrm{f}}(x)=\frac{\sum_{x_{i} \in \Omega} w_{i} P\left(\mathrm{f} \mid y_{i}\right) y_{i}}{\sum_{x_{i} \in \Omega} w_{i} P\left(\mathrm{f} \mid y_{i}\right)}
$$

where $P\left(\mathrm{f} \mid y_{i}\right)$ is provided by the classifiers.

Two measures are introduced to enable variable kernel sizes and address undefined reconstructions require: feature support, $\mathcal{F}_{\mathrm{f}}(x)$, and kernel validity, $\mathcal{V}_{\mathrm{f}}(x)$. Feature support is computed over a fixed size local neighborhood and is used to select an appropriate kernel size for each reconstruction. Kernel validity is derived from the strength of the normalization in Equation 4.4 and is used to adjust the visual impact of individual contributions, as well as to discard undefined reconstructions where the denominator of Equation 4.4 is near zero. For detailed formulations, the reader is referred to Paper VII.

The result of the variable kernel size is that standard-sized kernels are maintained within solid objects, while larger kernels are applied in boundary areas. This preserves high frequency information and also reduces the overall number of samples required by the approach.

Once signal estimates have been computed for all visualized features, these are mapped to visual contributions using their associated TF widgets. (The full TF is never applied to any of the estimates.) The visual contributions are then combined, weighted by their associated kernel validity. 


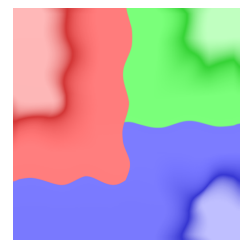

(a) Reference data.

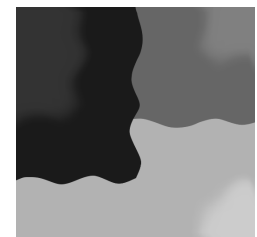

(b) TF mapped reference imge.

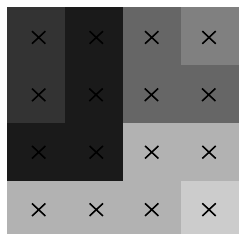

(c) Sampled data.

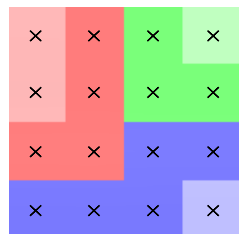

(d) Nearest neighbor color mapping.

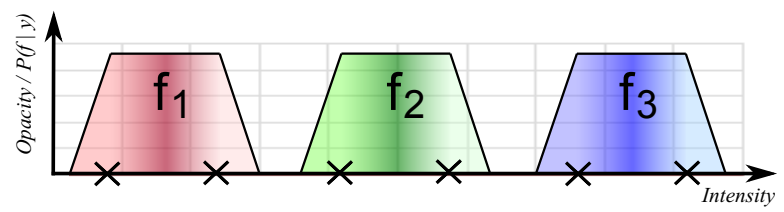

(e) Visual mapping and feature classification.

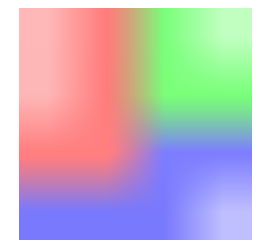

(f) Pre-class. DVR p.w. constant.

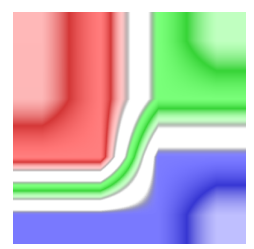

(g) Post-class DVR continuous.

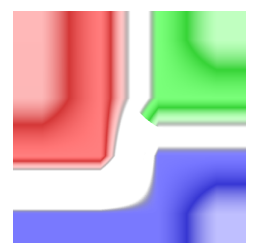

(h) Two-level DVR segmented.

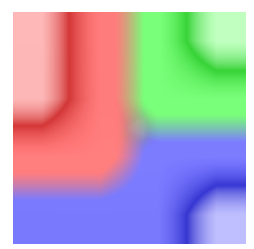

(i) Our method p.w. continuous.

$\Delta$ Figure 4.17 - The of using boundary aware reconstruction resembles postclassified DVR within features with fuzzy uncertainty representations in boundary areas.

\section{Method Results and Applicability}

The output of the method is compared to existing approaches in Figure 4.17 for a small synthetic data set. This example illustrates well the issues associated with existing methods. It also highlights how the output of the proposed method resembles post-classified DVR within features while fuzzy representations of uncertainty are created between features.

An example of applying the method to medical data is shown in Figure 4.18, with a magnification in 2D available in Figure 4.19. The data for this particular case were acquired with DECT, which entails an inherently 2D TF interface. The proposed method addresses the misclassifications without increasing the TF dimensionality. 

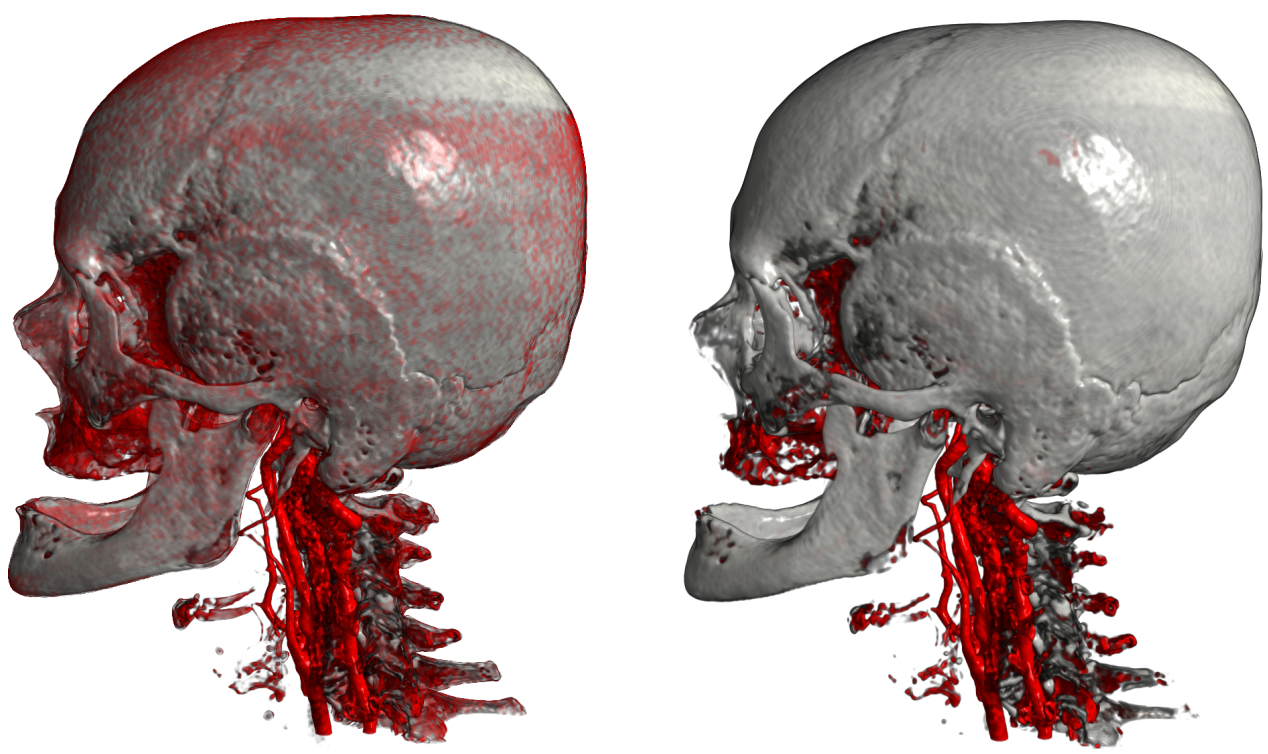

$\Delta$ Figure 4.18 - Dual-energy CT features improved material separability over standard CT, but does not prevent rendering artifacts (left). Artifacts can be removed by preventing undesirable reconstructions between features (right).
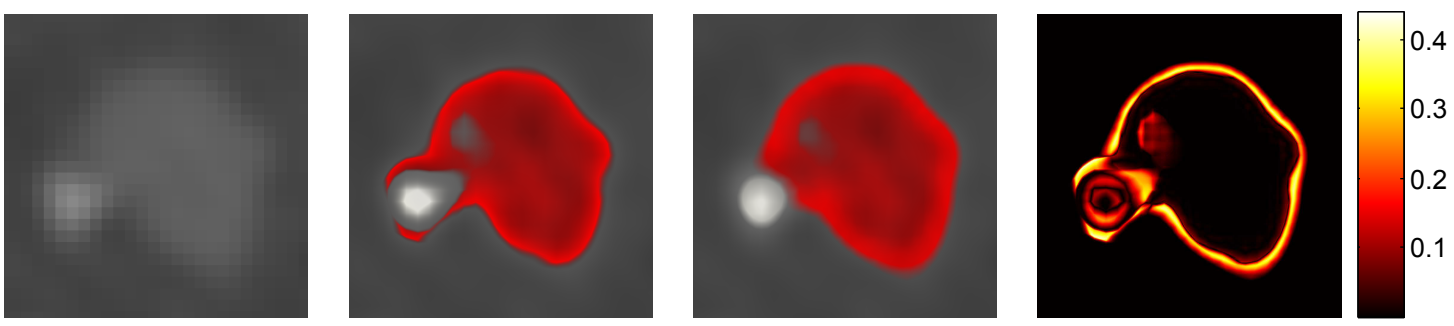

$\triangle$ Figure 4.19 - The slices show magnifications of one of the arteries in Figure 4.18. Left-to-right: the slice data, continuous interpolated data with TF mapping, the proposed piece-wise reconstructed data with TF mapping, and a difference image between the latter two. 




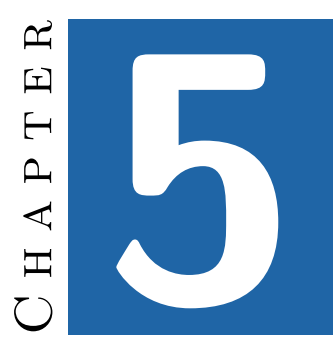

\section{Generalizations and Reflections}

Many interesting questions and discussions have taken place during the last five years of doctoral studies. This includes generalizations and discussions that do not fit into the format of scientific papers. Some of these are included here, starting with a set of discussions at a fairly technical level.

This chapter will also, on a less technical level, summarize this dissertation and its contributions. This will be accompanied by a selected set of reflections on both my personal research and the visualization community. I am afraid that from here on there will be more questions than answers, but such is ever the nature of science and research.

\subsection{Transfer Functions 3.0}

The act of associating semantic labels with TF widgets to identify targeted structures is a shared aspect of Paper III, IV, V \& VI. The action required by the user is, in itself, no more complicated than choosing an option from a right click menu. Yet the knowledge that is transferred, the identity of the targeted feature, opens many possibilities for tailored visualizations. The vesselness filters of Paper III are one example. Another example is the spatial conditions of Paper VI. The underlying generalization is, of course, that specialized tools and algorithms can be applied once the identity of the targeted feature is known.

This form of feature adaption is just one example of how the role of the traditional TF can be extended. Having an associated label also makes the 
60 Chapter 5 - Generalizations and Reflections

classification provided by the TF more explicit.

\subsubsection{Problematizing Transfer Function Classification}

Part of the work presented in Chapter 4 relies on the TF, or a TF-like interface, to supply feature classification. While this methodology is widespread in visualization, it is not the only one and neither is it entirely unproblematic.

Going back to Chapter 2, the role of the TF is described solely as a visual mapper, meaning that its only function is to assign visual properties to data values. In this interpretation, the TF has no probabilistic interpretation.

Yet as soon as a user designs a TF by assigning colors and opacities to perceived structures, there is some form of classification taking place. Something of which has been acknowledged in the literature:

"traditionally, the transfer function is not thought of as a feature classifier ... [with] volume visualization, however, transfer function design is going far beyond a simple color-table set-up"

Engel et al. [20]

"A fuzzy classification function ... [can] be employed to assign different colors and opacities to [observed structures in a histogram]"

Kaufman and Mueller [43]

This widens the role of the TF to, what can be described as, a visual classifier. In this interpretation, the opacity assigned by the TF to a certain data value is implicitly related to (but seldom properly formalized as) the probability that the sample point belongs to a certain feature. For example, the a common design for both control-point and widget based TFs has higher opacity near the expected value range for the targeted feature, with decreasing opacities further away. This decreasing opacity can be interpreted as the decreasing probability that the observed values belong to the targeted feature.

In the most stringent interpretation of the $\mathrm{TF}$, the assignment of visual attributes is paired with a set of proper probabilistic classifiers. This is the interpretation used in both Paper VI \& VII. As detailed in Chapter 4, the classifiers may be exposed as separate widgets or be derived from the visual mapping. However, interpreting user input in a probabilistic framework is not always straightforward. 


\subsubsection{Complications of Interpreting User Input}

One complication is that the user may not describe probability distributions for all features, but rather a few conditional probabilities for a smaller set of features of interest. This complication is addressed in Section 4.4 by the insertion of a null class, see Equation 4.2, p. 49. The use of a null class is inspired by the work of Lundström et al. [77], who use a null class for material likelihoods, $L_{m}(y)$, to extend the $M$ materials specified by the user

$$
\begin{aligned}
& L_{m}(y): \text { user supplied class likelihoods, } m \in[1, M] \\
& L_{0}(y)=\max \left(0.0,1.0-\sum_{m=1}^{M} L_{m}(y)\right) .
\end{aligned}
$$

Intensity specific material probabilities, $P_{m}(y)$, are then computed as normalized likelihoods

$$
P_{m}(y)=\frac{L_{m}(y)}{\sum_{m=0}^{M} L_{m}(y)} .
$$

It can be noted, however, that different forms of normalization are used in the work of Lundström et al. and Paper VII.

A related complication is the ambiguity of the interpretation of the classifiers as conditional probabilities. Possible interpretations include both class likelihoods, $P(y \mid C)$, and posterior probabilities, $P(C \mid y)$. Both Drebin et al. [17] and Lundström et al. [77] argues for likelihoods with the motivation that they should be derivable from observed material distributions. The former assume that likelihoods for all materials are known, while the latter includes a null class. It can be observed, however, that as long as $\sum_{m=1}^{M} L_{m}(y) \leq 1$ in Equation 5.2, the inclusion of the null class cancels out the denominator in Equation 5.3,

$$
P_{m}(y)=\frac{L_{m}(y)}{1.0-\sum_{m=1}^{M} L_{m}(y)+\sum_{m=1}^{M} L_{m}(y)}=L_{m}(y),
$$

resulting in an equivalence between likelihoods and posteriors. Normalization is thus only applied for intensities where the sum of likelihoods exceeds one. As a result, different parts of the user supplied estimates may be normalized differently.

To summarize the above, final probabilities are dependent both on the likelihood/posterior interpretation choice, as well as on any applied normalization. While the math can be solved for most cases, questions arise in relation to the user and his or her initial estimates. Is the user aware of which interpretation is being used? Would he/she understand the difference? And are certain normalizations more intuitive than others? 
A third complication of user provided classifiers is related to the user's goal of the interaction. This goal is likely to explore the data and achieve a meaningful visualization, not to maximize the correctness of any underlying probabilistic classifiers (regardless of their interpretation). This discrepancy arguably weakens any probabilistic interpretations of the TF. Yet, as was shown in Chapter 4, user provided classifiers can be quite powerful and desirable thanks to their accessibility and low costs. Another reason why probabilistic TFs could be valuable is found in the work of Lundström

"Another advantage of explicitly probabilistic TFs has been identified, which may prove to be an important evolution for clinical DVR ... During the evaluation of [work based on probabilistic TFs], several radiologists with expertise in DVR expressed that the classification mindset would be an important step towards more effective DVR interaction in diagnostic work."

Lundström [73]

\subsubsection{Problematizing Material Probability}

Another question not commonly discussed relates to what kind of probability classifiers actually express. In one interpretation, a posterior probability expresses "the probability of the spatial point $x$ belonging to class $C$, given the observed value $y^{\prime \prime}$, i.e. a spatial probability, or

$$
P(C \mid y) \equiv P(x \in C \mid y) .
$$

This interpretation is arguably the most common and seems to be implicitly applied in much of the literature.

In another interpretation, a posterior probability express "the probability of the observed value $y$ belonging to class $C$, given the observed value itself", i.e. a value probability, or

$$
P(C \mid y) \equiv P(y \in C \mid y) .
$$

This interpretation is arguably less common, but becomes important once the value is used in subsequent computations, such as in Paper VII.

The following example may clarify the difference between the two interpretations. Let some data contain air and bone with nominal values $y_{\text {air }} \sim-1000$ HU and $y_{\text {bone }} \sim 1000$ HU respectively. Then, let an observed value at point $x$ be $y(x)=50 \mathrm{HU}$ and compute the following probability: $P($ air $\mid y=50)$. With the spatial interpretation of the probability, $P(x \in$ air $\mid y=50) \sim 0.55$ is a reasonable 
output, which reads as a $55 \%$ chance that the point $x$ is occupied by air. With the value interpretation, a reasonable output may instead be $P(y \in$ air $\mid y=50) \sim 0.0$. The reason for this is that an observed value of 50 is highly unlikely to be a valid value for either pure air or pure bone. In fact, perhaps validity would be a better term for the entire concept.

This notion of validity shares many similarities to the radiometric weights used in bilateral filtering [116]. A similar concept is also found in the framework of Normalized Convolution [54], as a measure of certainty associated with signal samples. The concept has also been used for visualization purposes through moving least squares [60]. An extended classifier concept should likely include both spatial probability and signal validity.

\subsection{Summary and Future Work}

The work presented in Chapter 4 answers difficulties in an expanding and diverging visualization pipeline. An overall goal has been to improve medical volume visualization while maintaining sufficiently simple user interfaces. Many of the methods are therefore based on the traditional TF and its probabilistic interpretation. It has been shown that even the crude classification provided by a simple 1D interface can be useful in achieving effective data prioritization. This is particularly applicable for methods that operate on neighborhoods rather than on single voxel values. The information provided by the classifiers is used to both enable spatial conditions and to improve boundary representations. The latter method also goes beyond the traditional linear pipeline, using knowledge provided by the user already in the reconstruction stage. Again, the underlying idea is to maximize the use of information which is already in the system, i.e. the TF, so as to not introduce additional interaction complexity. The methodology of broadening the role of the TF is to no small degree inspired by the work of Lundström [73].

The improvements contained in this thesis can be summarized as follows:

- Fused visualization of volume-only and hybrid scenes has been made both simpler and faster through new data structures and improved memory management.

- Visualization of vessel trees has been improved with locally adaptive TFs, accounting for intensity shifts and studied in a clinical setting.

- Diagnostic volume rendering has been enriched with the ability to prioritize data through both anatomical ROIs and spatial material conditions.

- Representation of boundary regions have been addressed through a reconstruction approach with a minimal interface. 
In terms of algorithmical advancements, the introduction of feature adaptive reconstructions open up many interesting paths. For one, the kernel regression framework allows for reconstructions of any order, which would make gradient estimation a straightforward extension. At the same time, there is the option to study the changes in the feature support fields themselves. This would lead to the situation where one value, one data gradient and one feature gradient would be available per reconstruction point and feature. Such information has the potential to change the way we currently perform both illumination and highlighting.

A highly interesting direction for future work would, of course, be to answer some of the questions raised in Section 5.1 regarding the TF and its probabilistic interpretations. In particular, it would be interesting to study the users' understanding of the frameworks and their probabilistic aspects.

The integration of specialized versions of the presented methods in medical application would be a valuable direction for the future. While the manuscripts contain sample cases and potential areas of applicability, these would need to be investigated further. The methods also need to be included in the architecture ecosystems at clinical sites in order to effectively measure their utility. Unfortunately, this is not an easy task as one cannot simply install new software and expect the radiologists to use it. One possibility to circumvent this problem would be to provide the methods as augmentations to existing techniques, perhaps in the form of 'focus lenses'.

\subsection{Reflections}

As I finalize this thesis and reflect on my five+ years of research, there are many interesting trends that can be observed. One trend is the ever increasing number of arrows used whenever a visualization pipeline is illustrated. The narrow concept of a linear pipeline with a few fixed stages is going extinct. Two examples from this thesis alone are the addition of the prioritization stage and the utilization of user derived classifiers in the reconstruction stage. Going forward, the amount of auxiliary data will only increase as segmentation and registration algorithms become faster and more accurate. As this happens, the pipeline will likely become much more interconnected with flows of information in more than one direction. Significant research will likely need to be directed towards the consolidation of all this information and its varying degrees of accuracy.

A more interconnected pipeline also stresses the need to treat different parts of the visualization differently. In this thesis, a simple label added onto the 
TF was used to effectively prioritize data. Such specialization is likely to be integrated to the point where different aspects of the visualization would even have different pipeline illustrations.

A very much related trend is the interfaces exposed to the user. Current research is heavily focused on adapting the interface to the user, including many of the methods described in this thesis. Yet in the long run I believe the user will also adapt to the interface. Take the development of graphical tools such as Illustrator, Photoshop or Maya as examples. These highly technical and complicated interfaces are now common among illustrators and artists. Why could not radiologists go the same path? Sure the situations are very different, but as generations go by, the average radiologist may soon request interfaces far beyond what is provided today.

However, it is neither current achievements nor future technical strategies that stands out the most as I reflect on visualization and its community. Instead, what stands out is the possibility that the contributions contained in this thesis result in any real world impact. I say possibility, for while many of the manuscripts contain studies or evaluation that support their contributions to the visualization community, more work is certainly necessary before clinical deployment is feasible.

I believe evaluations is the key. I am not sure, however, if our community is the optimal community to perform them. If we are talking about evaluations that solidifies clinical value, then evaluations are expected to be quantitative, timed, task-based evaluations with a minimum of 20 or so participating domain experts. All of which costs significant resources. This in itself is not a problem, but it becomes a problem when the costs and risks associated with such studies are so significant that creating a new algorithm is always a cheaper and more efficient way to get a similarly valued publication. As a result, clinical deployment is often left as an exercise for the reader.

The industry fulfills the role of clinical validation to some degree, but I suspect that many possible contributions simply never make it to clinical environments. Going forward, I believe the visualization community needs to do its part in ensuring that more contributions are implemented in clinical workstations. Perhaps one way is to increase the prestige and recognition for evaluations and industry collaborations, such that their risk/reward ratios match that of more technical forms of research. Doing so would go a long way towards turning possibilities into reality and facilitate real world impacts, both for the work within the scope of this thesis as well as the community at large.

Stefan Lindholm 



\section{Bibliography}

[1] S. Andrews, G. Hamarneh, and A. Saad. Fast random walker with priors using precomputation for interactive medical image segmentation. In Medical Image Computing and Computer-Assisted Intervention (MICCAI), volume 6363 of Lecture Notes in Computer Science, pages 9-16, Beijing, China, 2010. Springer Berlin Heidelberg. doi:10.1007/978-3-642-15711-0_2. [page 33]

[2] K. P. Andriole, R. L. Morin, R. L. Arenson, J. A. Carrino, B. J. Erickson, S. C. Horii, D. W. Piraino, B. I. Reiner, J. A. Seibert, and E. Siegel. Addressing the coming radiology crisis-the society for computer applications in radiology transforming the radiological interpretation process (TRIP) initiative. Journal of Digital Imaging, 17(4):235-243, 2004. doi:10.1007/s10278004-1027-1. [page 21]

[3] K. P. Andriole, J. M. Wolfe, R. Khorasani, S. T. Treves, D. J. Getty, F. L. Jacobson, M. L. Steigner, J. J. Pan, A. Sitek, and S. E. Seltzer. Optimizing analysis, visualization, and navigation of large image data sets: One 5000-section CT scan can ruin your whole day. Radiology, 259(2):346-362, 2011. doi:10.1148/radiol.11091276. [page 21]

[4] L. Bavoil and K. Myers. Order-independent transparency with dual depth peeling. Technical report, NVIDIA Developer SDK 10, 2008. [page 25]

[5] L. Bavoil, S. P. Callahan, A. Lefohn, J. a. L. D. Comba, and C. T. Silva. Multi-fragment effects on the GPU using the $k$-buffer. In ACM SIGGRAPH Symposium on Interactive 3D Graphics and Games, pages 97-104, Seattle, Washington, 2007. doi:10.1145/1230100.1230117. [page 26]

[6] Å. Birkeland, V. Šoltészová, D. Hönigmann, O. H. Gilja, S. Brekke, T. Ropinski, and I. Viola. The ultrasound visualization pipeline - a survey. ArXiv e-prints, 1206.3975, 2012. [pages 8, 9, and 38]

[7] M. Brady. Image segmentation and classification. Lecture series on health informatics, 2005. [Online]. Available: http://www.robots.ox.ac.uk/ jmb/ lectures/InformaticsLecture5.pdf, University of Oxford. [page 8]

[8] R. Brecheisen, A. V. Bartroli, B. Platel, and B. M. ter Haar Romeny. Flexible GPU-based multi-volume ray-casting. In Vision, Modeling and Visualization, pages 303-312, Konstanz, Germany, 2008. [page 25] 
[9] S. Bruckner, S. Grimm, A. Kanitsar, and M. E. Gröller. Illustrative contextpreserving exploration of volume data. IEEE Transactions on Visualization and Computer Graphics, 12(6):1559-1569, 2006. doi:10.1109/TVCG.2006.96. [page 47]

[10] K. Bühler, P. Felkel, and A. L. Cruz. Geometric methods for vessel visualization and quantification - a survey. In Geometric Modelling for Scientific Visualization, Mathematics and Visualization, pages 399-420. Springer Berlin Heidelberg, 2003. doi:10.1007/978-3-662-07443-5_24. [page 31]

[11] W. Cai and G. Sakas. Data intermixing and multi-volume rendering. Computer Graphics Forum, 18(3):359-368, 1999. doi:10.1111/1467-8659.00356. [page 16]

[12] L. Carpenter. The A-buffer, an antialiased hidden surface method. In Computer Graphics, volume 18, pages 103-108, Minneapolis, USA, 1984. doi:10.1145/800031.808585. [page 26]

[13] N. Carr, R. Měch, and G. Miller. Coherent layer peeling for transparent high-depth-complexity scenes. In ACM SIGGRAPH/EUROGRAPHICS Workshop on Graphics Hardware, pages 33-40, Sarajevo, BosniaHerzegovina, 2008. doi:10.2312/EGGH/EGGH08/033-040. [page 25]

[14] C. D. Correa and K.-L. Ma. Size-based transfer functions: A new volume exploration technique. IEEE Transactions on Visualization and Computer Graphics, 14(6):1380-1387, 2008. doi:10.1109/TVCG.2008.162. [pages 27 and 29]

[15] C. Crassin. OpenGL 4.0+ ABuffer v2.0: Linked lists of fragment pages. Personal Blog, 2010. [Online]. Available: http://blog.icare3d.org/2010/07/, Open source OIT implementation. [page 26]

[16] S. Doyle, J. Monaco, A. Madabhushi, S. Lindholm, P. Ljung, L. Ladic, J. Tomaszewski, and M. Feldman. Evaluation of effects of JPEG2000 compression on a computer-aided detection system for prostate cancer on digitized histopathology. In IEEE International Symposium on Biomedical Imaging (ISBI), pages 1313-1316, Rotterdam, Netherlands, 2010. doi:10.1109/ISBI.2010.5490238. [page viii]

[17] R. A. Drebin, L. Carpenter, and P. Hanrahan. Volume rendering. In Computer Graphics, volume 22, pages 65-74. ACM, 1988. doi:10.1145/378456.378484. [page 61] 
[18] D. S. Ebert and R. E. Parent. Rendering and animation of gaseous phenomena by combining fast volume and scanline A-buffer techniques. In Computer Graphics, volume 24, pages 357-366, Dallas, USA, 1990. ACM. doi:10.1145/97880.97918. [page 26]

[19] K. Engel, M. Hadwiger, J. Kniss, A. E. Lefohn, C. Rezk-Salama, and D. Weiskopf. Real-time volume graphics. In ACM SIGGRAPH 2004 Course Notes, page 29. ACM, 2004. doi:10.1145/1103900.1103929. [pages 13 and 16]

[20] K. Engel, M. Hadwiger, J. M. Kniss, C. Rezk-Salama, and D. Weiskopf. Real-Time Volume Graphics. A. K. Peters, Ltd., 2006. [pages 14, 16, 23, and 60]

[21] C. Everitt. Interactive order-independent transparency. Technical report, NVIDIA, 2001, NVIDIA white paper. [page 25]

[22] R. Fernando. GPU Gems: Programming Techniques, Tips and Tricks for Real-Time Graphics. Pearson Higher Education, 2004. [page 14]

[23] I. Fovia. High definition volume rendering®. http://www.fovia.com/, 2014, Commersially available CPU based rendering engine. [page 24]

[24] A. Frangi, W. Niessen, K. Vincken, and M. Viergever. Multiscale vessel enhancement filtering. In Medical Image Computing and Computer-Assisted Interventation (MICCAI), volume 1496 of Lecture Notes in Computer Science, pages 130-137. Springer Berlin Heidelberg, 1998. doi:10.1007 /BFb0056195. [page 30]

[25] I. Fujishiro, T. Azuma, and Y. Takeshima. Automating transfer function design for comprehensible volume rendering based on 3D field topology. In Visualization, pages 467-563, San Francisco, USA, 1999. doi:10.1109/VISUAL.1999.809932. [page 28]

[26] S. Glaßer, S. Oeltze, A. Hennemuth, C. Kubisch, A. H. Mahnken, S. Wilhelmsen, and B. Preim. Automatic transfer function specification for visual emphasis of coronary artery plaque. Computer Graphics Forum, 29 (1):191-201, 2010. doi:10.1111/j.1467-8659.2009.01590.x. [page 31]

[27] R. C. Gonzalez and R. E. Woods. Digital Image Processing. Prentice-Hall, Inc., 3rd edition, 2006. [page 34]

[28] S. Grimm, S. Bruckner, A. Kanitsar, and M. E. Gröller. Flexible direct multi-volume rendering in interactive scenes. In Vision, Modeling and Visualization, pages 379-386, 2004. [page 24] 
[29] H. Guo and X. Yuan. Local WYSIWYG volume visualization. In IEEE Pacific Visualization Symposium, pages 65-72, 2013. doi:10.1109/PacificVis.2013.6596129. [page 29]

[30] H. Guo, N. Mao, and X. Yuan. WYSIWYG (what you see is what you get) volume visualization. IEEE Transactions on Visualization and Computer Graphics, 17(12):2106-2114, 2011. doi:10.1109/TVCG.2011.261. [page 29]

[31] M. Hadwiger, C. Berger, and H. Hauser. High-quality two-level volume rendering of segmented data sets on consumer graphics hardware. In IEEE Visualization, VIS '03, pages 301-308, Washington, DC, USA, 2003. IEEE Computer Society. doi:10.1109/VISUAL.2003.1250386. [pages 28, 33, and 47]

[32] M. Hadwiger, F. Laura, C. Rezk-Salama, T. Höllt, G. Geier, and T. Pabel. Interactive volume exploration for feature detection and quantification in industrial CT data. IEEE Transactions on Visualization and Computer Graphics, 14(6):1507-1514, 2008. doi:10.1109/TVCG.2008.147. [page 29]

[33] M. Haidacher, D. Patel, S. Bruckner, A. Kanitsar, and M. E. Gröller. Volume visualization based on statistical transfer-function spaces. In IEEE Pacific Visualization Symposium, pages 17-24, Taipei, Taiwan, 2010. doi:10.1109/PACIFICVIS.2010.5429615. [page 27]

[34] H. Hauser, L. Mroz, G. I. Bischi, and E. Gröller. Two-level volume rendering. IEEE Transactions on Visualization and Computer Graphics, 7(3):242-252, 2001. doi:10.1109/2945.942692. [pages 28 and 47]

[35] T. He, L. Hong, A. Kaufman, and H. Pfister. Generation of transfer functions with stochastic search techniques. In IEEE Visualization, page 227, San Francisco, USA, 1996. IEEE. doi:10.1109/VISUAL.1996.568113. [page 30]

[36] C. Heinzl, J. Kastner, T. Möller, and M. E. Gröller. Statistical analysis of multi-material components using dual energy CT. In Vision, Modeling and Visualization, pages 179-188, Konstanz, Germany, 2008. [page 33]

[37] Y. Hu and R. A. Malthaner. The feasibility of three-dimensional displays of the thorax for preoperative planning in the surgical treatment of lung cancer. European journal of cardio-thoracic surgery, 31(3):506-511, 2007. doi:10.1016/j.ejcts.2006.11.054. [page 28]

[38] M. Ikits, J. Kniss, A. E. Lefohn, and C. Hansen. GPU Gems: Volume Rendering Techniques, chapter 39. Addison-Wesley Professional, 2004. [page 14] 
[39] C. Y. Ip, A. Varshney, and J. Jaja. Hierarchical exploration of volumes using multilevel segmentation of the intensity-gradient histograms. IEEE Transactions on Visualization and Computer Graphics, 18(12):2355-2363, 2012. doi:10.1109/TVCG.2012.231. [page 29]

[40] C. Johnson and C. Hansen, editors. The Visualization Handbook. Academic Press, Inc., 1 edition, 2004. [pages 14 and 23]

[41] A. Joshi, X. Qian, D. Dione, K. Bulsara, C. Breuer, A. Sinusas, and X. Papademetris. Effective visualization of complex vascular structures using a non-parametric vessel detection method. IEEE Transactions on Visualization and Computer Graphics, 14(6):1603-1610, 2008. doi:10.1109/TVCG.2008.123. [page 30]

[42] B. Kainz, M. Grabner, A. Bornik, S. Hauswiesner, J. Muehl, and D. Schmalstieg. Ray casting of multiple volumetric datasets with polyhedral boundaries on manycore GPUs. ACM Transactions on Graphics, 28(5):152:1-152:9, 2009. doi:10.1145/1618452.1618498. [page 26]

[43] A. Kaufman and K. Mueller. The Visualization Handbook, chapter Overview of Volume Rendering, pages 127-174. Elsevier, 2005. [page 60]

[44] D. Kauker, M. Krone, A. Panagiotidis, G. Reina, and T. Ertl. Rendering molecular surfaces using order-independent transparency. In Eurographics Symposium on Parallel Graphics and Visualization, volume 13, pages 33-40, Girona, Spain, 2013. doi:10.2312/EGPGV /EGPGV13/033-040. [page 25]

[45] T. Kerwin, B. Hittle, H.-W. Shen, D. Stredney, and G. Wiet. Anatomical volume visualization with weighted distance fields. In Eurographics Workshop on Visual Computing for Biology and Medicine (VCBM), volume Vi, pages 117-124, 2010. doi:10.2312/VCBM/VCBM10/117-124. [pages 28, 33, and 47]

[46] E. Kerzner, C. Wyman, L. Butler, and C. Gribble. Toward efficient and accurate order-independent transparency. In ACM SIGGRAPH 2013 Posters, SIGGRAPH '13, pages 109:1-109:1, Anaheim, USA, 2013. ACM. doi:10.1145/2503385.2503504. [page 26]

[47] R. Khlebnikov, B. Kainz, M. Steinberger, and D. Schmalstieg. Noise-based volume rendering for the visualization of multivariate volumetric data. IEEE Transactions on Visualization and Computer Graphics, 19(12):2926-2935, 2013. doi:10.1109/TVCG.2013.180. [page 47] 
[48] G. Kindlmann and J. W. Durkin. Semi-automatic generation of transfer functions for direct volume rendering. In IEEE Symposium on Volume Visualization, pages 79-86, Research Triangle Park, USA, 1998. IEEE. doi:10.1109/SVV.1998.729588. [pages 27 and 33]

[49] C. Kirbas and F. Quek. A review of vessel extraction techniques and algorithms. ACM Computing Surveys, 36(2):81-121, 2004. doi:10.1145/1031120.1031121. [page 31]

[50] J. Kniss, G. Kindlmann, and C. Hansen. Interactive volume rendering using multi-dimensional transfer functions and direct manipulation widgets. IEEE Transactions on Visualization and Computer Graphics, pages 255-262, 2001. doi:10.1109/VISUAL.2001.964519. [page 27]

[51] J. Kniss, G. Kindlmann, and C. Hansen. Multidimensional transfer functions for interactive volume rendering. IEEE Transactions on Visualization and Computer Graphics, 8(3):270-285, 2002. doi:10.1109/TVCG.2002.1021579. [pages 27 and 33]

[52] J. Kniss, S. Premoze, M. Ikits, A. E. Lefohn, C. Hansen, and E. Praun. Gaussian transfer functions for multi-field volume visualization. IEEE Transactions on Visualization and Computer Graphics, page 65, 2003. doi:10.1109/VISUAL.2003.1250412. [page 28]

[53] J. Kniss, R. Van Uitert, A. Stephens, G. Li, T. Tasdizen, and C. Hansen. Statistically quantitative volume visualization. In IEEE Visualization, pages 287-294, Baltimore, USA, 2005. IEEE. doi:10.1109/VIS.2005.91. [page 34]

[54] H. Knutsson and C.-F. Westin. Normalized and differential convolution: Methods for interpolation and filtering of incomplete and uncertain data. In Computer Vision and Pattern Recognition, pages 515-523, 1993. [page 63]

[55] D. H. Laidlaw. Geometric Model Extraction from Magnetic Resonance Volume Data. PhD thesis, California Institute of Technology, 1995. [page 34]

[56] D. H. Laidlaw, K. W. Fleischer, and A. H. Barr. Partial-volume bayesian classification of material mixtures in MR volume data using voxel histograms. IEEE Transactions on Medical Imaging, 17(1):74-86, 1998. doi:10.1109/42.668696. [page 33]

[57] G. Läthén. Level Set Segmentation and Volume Visualization of Vascular Trees. PhD thesis, Linköping University, 2013. [pages 31 and 44] 
[58] G. Läthén, S. Lindholm, R. Lenz, A. Persson, and M. Borga. Automatic tuning of spatially varying transfer functions for blood vessel visualization. IEEE Transactions on Visualization and Computer Graphics, 18(12): 2345-2354, 2012. doi:10.1109/TVCG.2012.203. [page 30]

[59] G. Läthén, S. Lindholm, R. Lenz, and M. Borga. Evaluation of transfer function methods in direct volume rendering of the blood vessel lumen. In Eurographics Workshop on Visual Computing for Biology and Medicine (VCBM), Vienna, Austria, 2014. The Eurographics Association, In press.

[60] C. Ledergerber, G. Guennebaud, M. Meyer, M. Bacher, and H. Pfister. Volume MLS ray casting. IEEE Transactions on Visualization and Computer Graphics, 14(6):1539-1546, 2008. doi:10.1109/TVCG.2008.186. [page 63]

[61] D. Lesage, E. D. Angelini, I. Bloch, and G. Funka-Lea. A review of 3D vessel lumen segmentation techniques: Models, features and extraction schemes. Medical Image Analysis, 13(6):819-845, 2009. doi:10.1016/j.media.2009.07.011. [page 31]

[62] M. Levoy. Display of surfaces from volume data. IEEE Computer Graphics and Applications, 8(3):29-37, 1988. doi:10.1109/38.511. [pages 27 and 33]

[63] B. Lichtenbelt, R. Crane, and S. Naqvi. Introduction to Volume Rendering. Prentice Hall, 1998. [page 14]

[64] S. Lindholm and A. Bock. Poor man's rendering of segmented data. In SIGRAD, pages 19-54, Norrköping, Sweden, 2013. [page viii]

[65] S. Lindholm and J. Kronander. Accounting for uncertainty in medical data: A CUDA implementation of normalized convolution. In SIGRAD, pages 35-42, Stockholm, Sweden, 2011. [page viii]

[66] S. Lindholm, P. Ljung, M. Hadwiger, and A. Ynnerman. Fused multivolume DVR using binary space partitioning. Computer Graphics Forum, 28(3):847-854, 2009. doi:10.1111/j.1467-8659.2009.01465.x. [page 24]

[67] S. Lindholm, P. Ljung, C. Lundström, A. Persson, and A. Ynnerman. Spatial conditioning of transfer functions using local material distributions. IEEE Transactions on Visualization and Computer Graphics, 16(6):1301-1310, 2010. doi:10.1109/TVCG.2010.195.

[68] S. Lindholm, D. Jönsson, H. Knutsson, and A. Ynnerman. Towards coherent sampling in volume rendering using iso surfaces. In SIGRAD, pages 55-60, Norrköping, Sweden, 2013. [page viii] 
[69] S. Lindholm, M. Falk, E. Sundén, A. Bock, A. Ynnerman, and T. Ropinski. Hybrid data visualization based on depth complexity histograms. Computer Graphics Forum, 2014. doi:10.1111/cgf.12460, In press.

[70] S. Lindholm, D. Forsberg, A. Ynnerman, H. Knutsson, M. Andersson, and C. Lundström. Towards clinical deployment of automated anatomical regions-of-interest. In Eurographics Workshop on Visual Computing for Biology and Medicine (VCBM), Vienna, Austria, 2014. The Eurographics Association, In press.

[71] S. Lindholm, D. Jönsson, C. Hansen, and A. Ynnerman. Boundary aware reconstruction of scalar fields. IEEE Transactions on Visualization and Computer Graphics, 20(4), 2014. doi:10.1109/TVCG.2014.2346351, In press.

[72] F. Liu, M.-C. Huang, X.-H. Liu, and E.-H. Wu. Efficient depth peeling via bucket sort. In Conference on High Performance Graphics, pages 51-57, New Orleans, USA, 2009. doi:10.1145/1572769.1572779. [page 25]

[73] C. Lundström. Efficient Medical Volume Visualization: An Approach Based on Domain Knowledge. PhD thesis, Linköping University, Department of Science and Technology, The Institute of Technology, 2007. [pages 62 and 63]

[74] C. Lundström and A. Persson. Characterizing visual analytics in diagnostic imaging. In International Workshop on Visual Analytics (EuroVA), pages 1-4, Bergen, Norway, 2011. Eurographics Association. doi:10.2312/PE/EuroVAST/EuroVA11/001-004. [page 21]

[75] C. Lundström, P. Ljung, and A. Ynnerman. Extending and simplifying transfer function design in medical volume rendering using local histograms. Computer Graphics Forum, pages 263-270, 2005. doi:10.2312/VisSym/EuroVis05/263-270. [pages 27 and 29]

[76] C. Lundström, P. Ljung, and A. Ynnerman. Local histograms for design of transfer functions in direct volume rendering. IEEE Transactions on Visualization and Computer Graphics, 12(6):1570-9, 2006. doi:10.1109/TVCG.2006.100. [pages 27 and 29]

[77] C. Lundström, P. Ljung, A. Persson, and A. Ynnerman. Uncertainty visualization in medical volume rendering using probabilistic animation. IEEE Transactions on Visualization and Computer Graphics, 13(6):1648-1655, 2007. doi:10.1109/TVCG.2007.70518. [pages 34 and 61] 
[78] C. Lundström, A. Persson, S. Ross, P. Ljung, S. Lindholm, F. Gyllensvärd, and A. Ynnerman. State-of-the-art of visualization in post-mortem imaging. Acta Pathologica (APMIS), 120(4):316-326, 2012. doi:10.1111/j.16000463.2011.02857.x. [page viii]

[79] C. Lux and B. Fröhlich. GPU-based ray-casting of multiple multiresolution volume datasets. In Advances in Visual Computing, volume 5876 of Lecture Notes in Computer Science, pages 104-116. Springer Berlin Heidelberg, 2009. doi:10.1007/978-3-642-10520-3_10. [pages 24 and 25]

[80] R. Maciejewski, I. Woo, W. Chen, and D. S. Ebert. Structuring feature space: A non-parametric method for volumetric transfer function generation. IEEE Transactions on Visualization and Computer Graphics, 15(6): 1473-1480, 2009. doi:10.1109/TVCG.2009.185. [page 29]

[81] A. Mammen. Transparency and antialiasing algorithms implemented with the virtual pixel maps technique. IEEE Computer Graphics and Applications, 9(4):43-55, 1989. doi:10.1109/38.31463. [page 25]

[82] J. Marks, B. Andalman, P. A. Beardsley, W. Freeman, S. Gibson, J. Hodgins, T. Kang, B. Mirtich, H. Pfister, W. Ruml, K. Ryall, J. Seims, and S. Shieber. Design galleries: a general approach to setting parameters for computer graphics and animation. In SIGGRAPH, pages 389-400, Los Angeles, USA, 1997. ACM Press/Addison-Wesley Publishing Co. doi:10.1145/258734.258887. [page 30]

[83] M. Maule, J. Comba, R. Torchelsen, and R. Bastos. Memory-efficient orderindependent transparency with dynamic fragment buffer. In Graphics, Patterns and Images (SIBGRAPI), pages 134-141, Ouro Preto, Brazil, 2012. doi:10.1109/SIBGRAPI.2012.27. [page 26]

[84] N. Max. Optical models for direct volume rendering. IEEE Transactions on Visualization and Computer Graphics, 1(2):99-108, 1995. doi:10.1109/2945.468400. [page 14]

[85] A. Morar, F. Moldoveanu, and M. E. Gröller. Image segmentation based on active contours without edges. In IEEE International Conference on Intelligent Computer Communication and Processing (ICCP), pages 213-220, 2012. doi:10.1109/ICCP.2012.6356188. [page 33]

[86] K. Myers and L. Bavoil. Stencil routed A-buffer. In ACM SIGGRAPH 2007 Sketches, San Diego, USA, 2007. doi:10.1145/1278780.1278806. [page 26] 
[87] B. F. Naylor, J. Amanatides, and W. Thibault. Merging BSP trees yields polyhedral set operations. In Computer Graphics, volume 24(4), pages 115-124, aug, 1990. doi:10.1145/97880.97892. [page 39]

[88] D. Patel, M. Haidacher, J.-P. Balabanian, and M. E. Gröller. Moment curves. In IEEE Pacific Visualization Symposium, pages 201-208, Peking, China, 2009. doi:10.1109/PACIFICVIS.2009.4906857. [page 27]

[89] H. Pfister, B. Lorensen, C. Bajaj, G. Kindlmann, W. Schroeder, L. S. Avila, K. Martin, R. Machiraju, and J. Lee. The transfer function bake-off. IEEE Computer Graphics and Applications, 21(3):16-22, 2001. doi:10.1109/38.920623. [page 27]

[90] J. Plate, T. Holtkaemper, and B. Froehlich. A flexible multi-volume shader framework for arbitrarily intersecting multi-resolution datasets. IEEE Transactions on Visualization and Computer Graphics, 13(6):1584-91, 2007. doi:10.1109/TVCG.2007.70534. [pages 24 and 25]

[91] J.-S. Prassni, T. Ropinski, J. Mensmann, and K. Hinrichs. Shape-based transfer functions for volume visualization. In IEEE Pacific Visualization Symposium, pages 9-16, 2010. doi:10.1109/PACIFICVIS.2010.5429624. [pages 27 and 29]

[92] B. Preim and D. Bartz. Visualization in Medicine: Theory, Algorithms, and Applications. Morgan Kaufmann Publishers Inc., San Francisco, CA, USA, 2007. [pages 7, 23, and 31]

[93] B. Preim and S. Oeltze. 3D visualization of vasculature: an overview. In Visualization in Medicine and Life Sciences, Mathematics and Visualization, pages 39-59. Springer Berlin Heidelberg, 2008. doi:10.1007/978-3-54072630-2_3. [page 30]

[94] P. Rautek, S. Bruckner, and M. E. Gröller. Semantic layers for illustrative volume rendering. IEEE Transactions on Visualization and Computer Graphics, 13(6):1336-1343, 2007. doi:10.1109/TVCG.2007.70591. [pages 29 and 47]

[95] P. Rautek, S. Bruckner, E. Gröller, and I. Viola. Illustrative visualization: New technology or useless tautology? ACM SIGGRAPH Computer Graphics, 42(3):4:1-4:8, 2008. doi:10.1145/1408626.1408633. [page 47]

[96] P. Rautek, S. Bruckner, and M. E. Gröller. Interaction-dependent semantics for illustrative volume rendering. Computer Graphics Forum, 27(3):847-854, 2008. doi:10.1111/j.1467-8659.2008.01216.x. [page 29] 
[97] B. Reiner. One size (doesn't) fit all. Journal of the American College of Radiology, 5(4):567 - 570, 2008. doi:10.1016/j.jacr.2007.09.006. [page 21]

[98] B. Reitinger, A. Bornik, R. Beichel, and D. Schmalstieg. Liver surgery planning using virtual reality. IEEE Computer Graphics and Applications, 26 (6):36-47, 2006. doi:10.1109/MCG.2006.131. [page 28]

[99] C. Rezk-Salama, M. Keller, and P. Kohlmann. High-level user interfaces for transfer function design with semantics. IEEE Transactions on Visualization and Computer Graphics, 12(5):1021-1028, 2006. doi:10.1109/TVCG.2006.148. [page 29]

[100] F. Rössler, E. Tejada, T. Fangmeier, T. Ertl, and M. Knauff. GPU-based multi-volume rendering for the visualization of functional brain images. In Simulation und Visualisierung (SimVis), pages 305-318, 2006. [page 24]

[101] F. Rössler, R. Botchen, and T. Ertl. Dynamic shader generation for GPUbased multi-volume ray casting. IEEE Computer Graphics and Applications, 28(5):66-77, 2008. doi:10.1109/MCG.2008.96. [page 25]

[102] S. Röttger, M. Bauer, and M. Stamminger. Spatialized transfer functions. In Eurographics / IEEE VGTC Symposium on Visualization (EuroVis), pages 271-278, Leeds, United Kingdom, 2005. Eurographics Association. doi:10.2312/VisSym/EuroVis05/271-278. [page 27]

[103] A. Saad, G. Hamarneh, and T. Möller. Exploration and visualization of segmentation uncertainty using shape and appearance prior information. IEEE Transactions on Visualization and Computer Graphics, 16(6):1366-75, 2010. doi:10.1109/TVCG.2010.152. [page 34]

[104] P. Santago and H. Gage. Statistical models of partial volume effect. IEEE Transactions on Image Processing, 4(11):1531-1540, 1995. doi:10.1109/83.469934. [page 34]

[105] Y. Sato, S. Nakajima, N. Shiraga, H. Atsumi, S. Yoshida, T. Koller, G. Gerig, and R. Kikinis. Three-dimensional multi-scale line filter for segmentation and visualization of curvilinear structures in medical images. Medical Image Analysis, 2(2):143-168, 1998. doi:10.1016/S1361-8415(98)80009-1. [page 45]

[106] Y. Sato, C.-F. Westin, A. Bhalerao, S. Nakajima, N. Shiraga, S. Tamura, and R. Kikinis. Tissue classification based on 3D local intensity structure for volume rendering. IEEE Transactions on Visualization and Computer Graphics, 6(2):160-180, 2000. doi:10.1109/2945.856997. [pages 27 and 30] 
[107] P. Sereda, A. Bartroli, I. W. O. Serlie, and F. Gerritsen. Visualization of boundaries in volumetric data sets using LH histograms. IEEE Transactions on Visualization and Computer Graphics, 12(2):208-218, 2006. doi:10.1109/TVCG.2006.39. [page 27]

[108] M. Shiozawa, N. Sata, K. Endo, M. Koizumi, Y. Yasuda, H. Nagai, and H. Takakusaki. Preoperative virtual simulation of adrenal tumors. Abdominal Imaging, 34(1):113-120, 2009. doi:10.1007/s00261-008-9364-z. [page 28]

[109] N. A. Svakhine, D. S. Ebert, and D. Stredney. Illustration motifs for effective medical volume illustration. IEEE Computer Graphics and Applications, 25(3):31-39, 2005. doi:10.1109/MCG.2005.60. [page 29]

[110] S. Takahashi, Y. Takeshima, and I. Fujishiro. Topological volume skeletonization and its application to transfer function design. Graphical Models, 66(1):24-49, 2004. doi:10.1016/j.gmod.2003.08.002. [page 28]

[111] Y. Takeshima, S. Takahashi, I. Fujishiro, and G. M. Nielson. Introducing topological attributes for objective-based visualization. In IEEE Visualization. IEEE, 2004. doi:10.1109/VISUAL.2004.57. [page 28]

[112] A. Tappenbeck, B. Preim, and V. Dicken. Distance-based transfer function design: Specification methods and applications. In Simulation und Visualisierung (SimVis), pages 259-274, Magdeburg, Germany, 2006. SCS Publishing House e.V. [pages 28 and 47]

[113] S. Tenginakai and R. Machiraju. Statistical computation of salient ISOvalues. In Symposium on Data Visualisation (VISSYM), pages 19-24, Barcelona, Spain, 2002. Eurographics Association. [page 27]

[114] S. Tenginakai, J. Lee, and R. Machiraju. Salient ISO-surface detection with model-independent statistical signatures. In Visualization, pages 231-238, San Diego, USA, 2001. doi:10.1109/VISUAL.2001.964516. [page 27]

[115] U. Tiede, T. Schiemann, and K. Hohne. High quality rendering of attributed volume data. In Visualization, pages 255-262, 1998. doi:10.1109/VISUAL.1998.745311. [page 33]

[116] C. Tomasi and R. Manduchi. Bilateral filtering for gray and color images. In Computer Vision, pages 839-846, Mumbai, India, 1998. IEEE. doi:10.1109/VISUAL.2004.57. [page 63]

[117] F.-Y. Tzeng, E. B. Lum, and K.-L. Ma. A novel interface for higherdimensional classification of volume data. In IEEE Visualization, pages 505-512, Seattle, USA, 2003. doi:10.1109/VISUAL.2003.1250413. [page 29] 
[118] A. Vasilakis and I. Fudos. S-buffer: Sparsity-aware multi-fragment rendering. In C. Andújar and E. Puppo, editors, Eurographics - Short Papers, pages 101-104, Cagliari, Italy, 2012. Eurographics Association. doi:10.2312/conf/EG2012/short/101-104. [page 26]

[119] A. A. Vasilakis and I. Fudos. $k^{+}$-buffer: Fragment synchronized kbuffer. In ACM SIGGRAPH Symposium on Interactive 3D Graphics and Games, I3D '14, pages 143-150, San Francisco, USA, 2014. ACM. doi:10.1145/2556700.2556702. [page 26]

[120] I. Viola, A. Kanitsar, and M. E. Gröller. Importance-driven volume rendering. In IEEE Visualization, pages 139-145, Austin, USA, 2004. IEEE. doi:10.1109/VISUAL.2004.48. [page 29]

[121] G. H. Weber, S. E. Dillard, H. Carr, V. Pascucci, and B. Hamann. Topologycontrolled volume rendering. IEEE Transactions on Visualization and Computer Graphics, 13(2):330-341, 2007. doi:10.1109/TVCG.2007.47. [page 28]

[122] M. A. Westenberg, J. Roerdink, and M. H. F. Wilkinson. Volumetric attribute filtering and interactive visualization using the max-tree representation. IEEE Transactions on Image Processing, 16(12):2943-2952, 2007. doi:10.1109/TIP.2007.909317. [page 28]

[123] Y. $\mathrm{Wu}$ and $\mathrm{H}$. Qu. Interactive transfer function design based on editing direct volume rendered images. IEEE Transactions on Visualization and Computer Graphics, 13(5):1027-1040, 2007. doi:10.1109/TVCG.2007.1051. [page 30]

[124] J. C. Yang, J. Hensley, H. Grün, and N. Thibieroz. Real-time concurrent linked list construction on the GPU. Computer Graphics Forum, 29(4): 1297-1304, 2010. doi:10.1111/j.1467-8659.2010.01725.x. [pages 25 and 26]

[125] L. Zhou, M. Schott, and C. Hansen. Transfer function combinations. Computers \& Graphics, 36(6):596-606, 2012. doi:10.1016/j.cag.2012.02.007, 2011 Joint Symposium on Computational Aesthetics (CAe), Non-Photorealistic Animation and Rendering (NPAR), and Sketch-Based Interfaces and Modeling (SBIM). [page 29] 


\section{Articles}

The articles associated with this thesis have been removed for copyright reasons. For more details about these see:

http://urn.kb.se/resolve?urn=urn:nbn:se:liu:diva-110239 


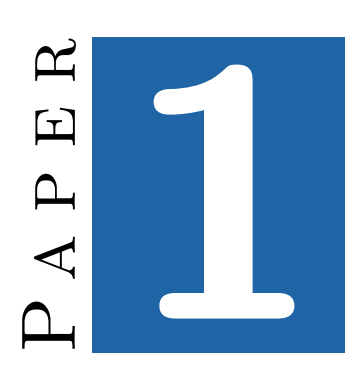

Fused Multi-Volume DVR using Binary Space Partitioning

(C) 2009 The Eurographics Association and John Wiley \& Sons Ltd.

S. Lindholm, P. Ljung, M. Hadwiger, and A. Ynnerman. Fused multivolume DVR using binary space partitioning. Computer Graphics Forum, 28(3):847-854, 2009. doi:10.1111/j.1467-8659.2009.01465.x. 


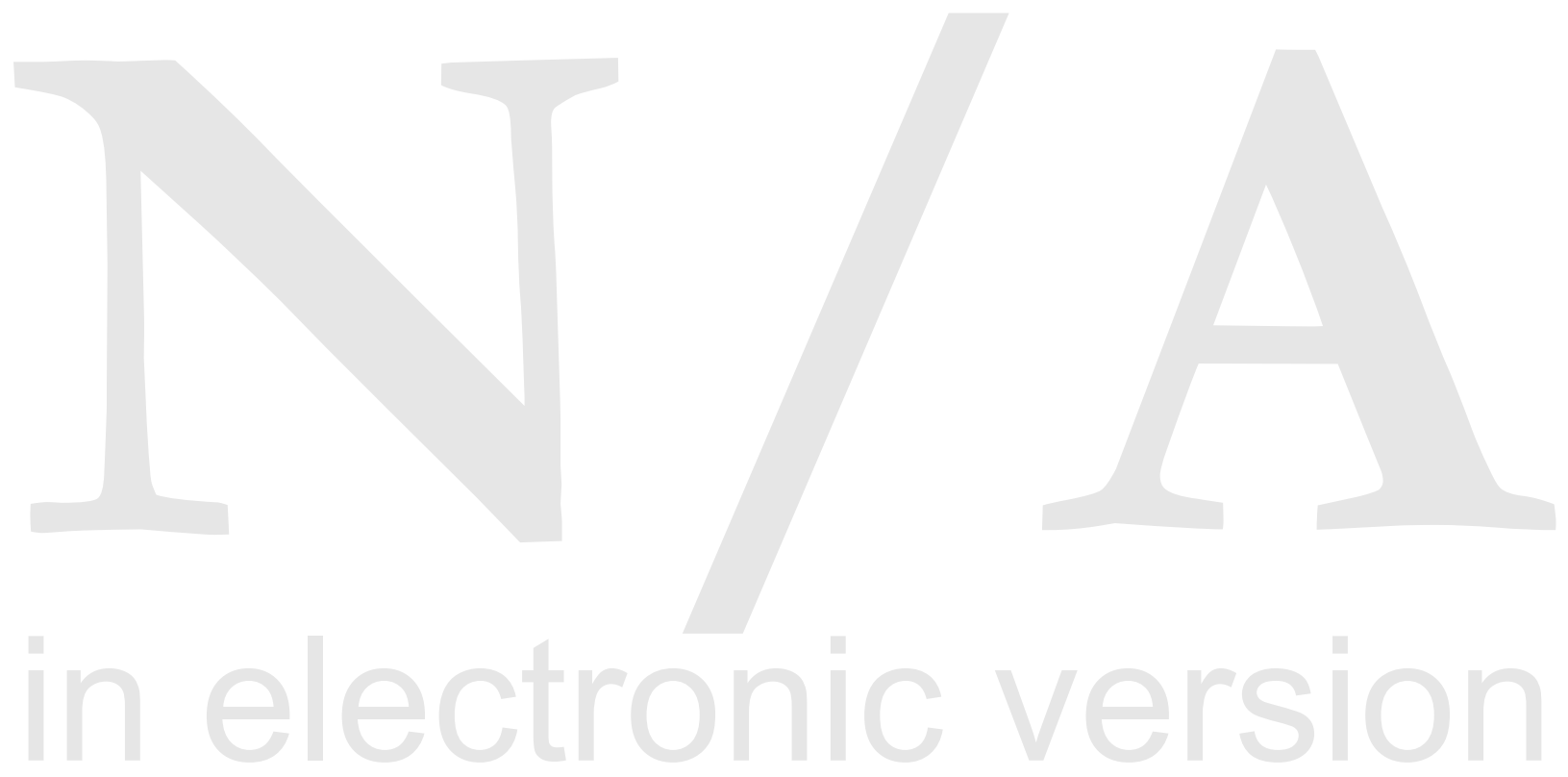




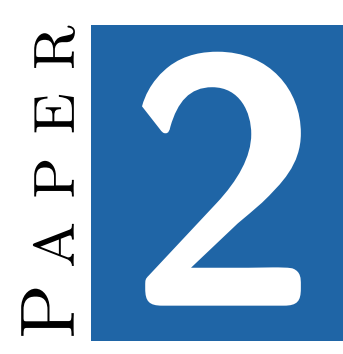

\section{Hybrid Data Visualization Based On Depth Complexity Histograms}

(C) 2014 The Eurographics Association and John Wiley \& Sons Ltd.

S. Lindholm, M. Falk, E. Sundén, A. Bock, A. Ynnerman, and T. Ropinski. Hybrid data visualization based on depth complexity histograms. Computer Graphics Forum, 2014. doi:10.1111/cgf.12460, In press. 


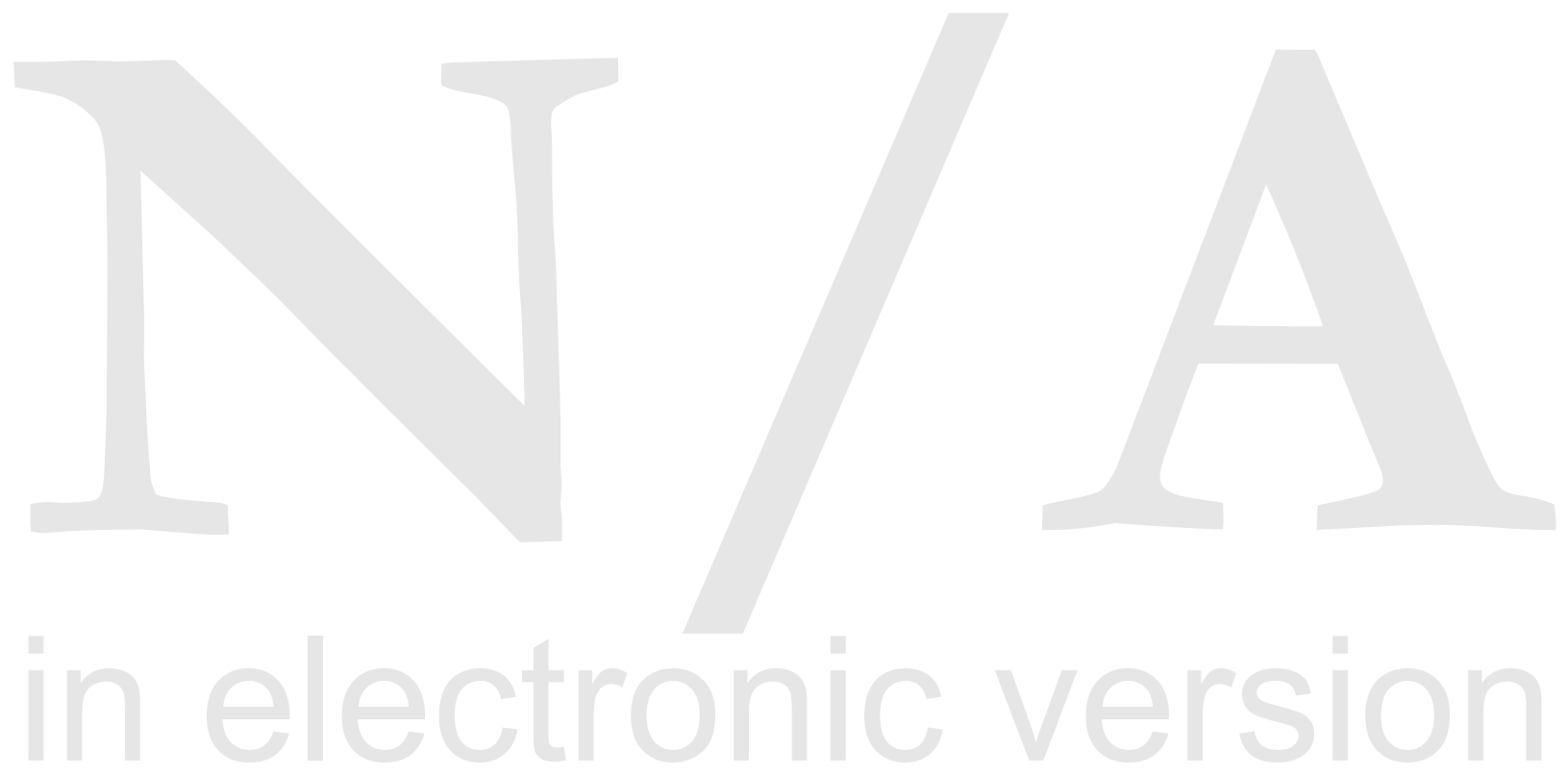


Automatic Tuning of Spatially Varying Transfer Functions for Blood Vessel Visualization

(C) 2012 IEEE Computer Society.

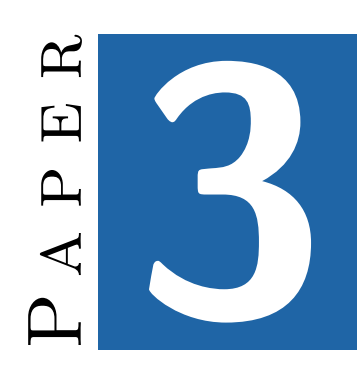

G. Läthén, S. Lindholm, R. Lenz, A. Persson, and M. Borga. Automatic tuning of spatially varying transfer functions for blood vessel visualization. IEEE Transactions on Visualization and Computer Graphics, 18(12): 2345-2354, 2012. doi:10.1109/TVCG.2012.203. 


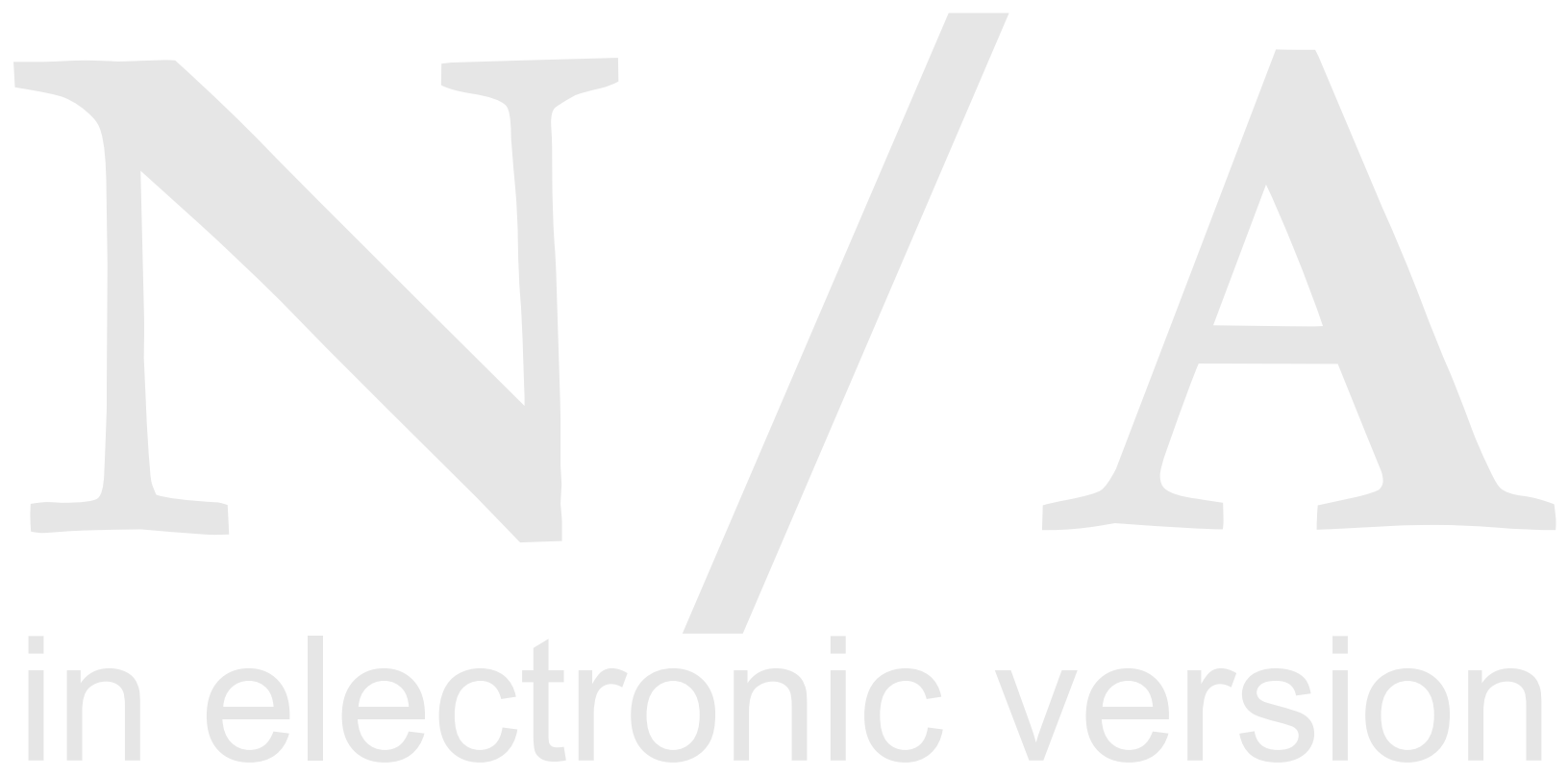


Evaluation of Transfer Function Methods in Direct Volume Rendering of the Blood Vessel Lumen

(C) 2014 The Eurographics Association.

G. Läthén, S. Lindholm, R. Lenz, and M. Borga. Evaluation of transfer function methods in direct volume rendering of the blood vessel lumen. In Eurographics Workshop on Visual Computing for Biology and Medicine (VCBM), Vienna, Austria, 2014. The Eurographics Association, In press.

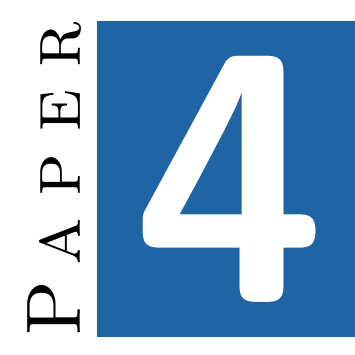




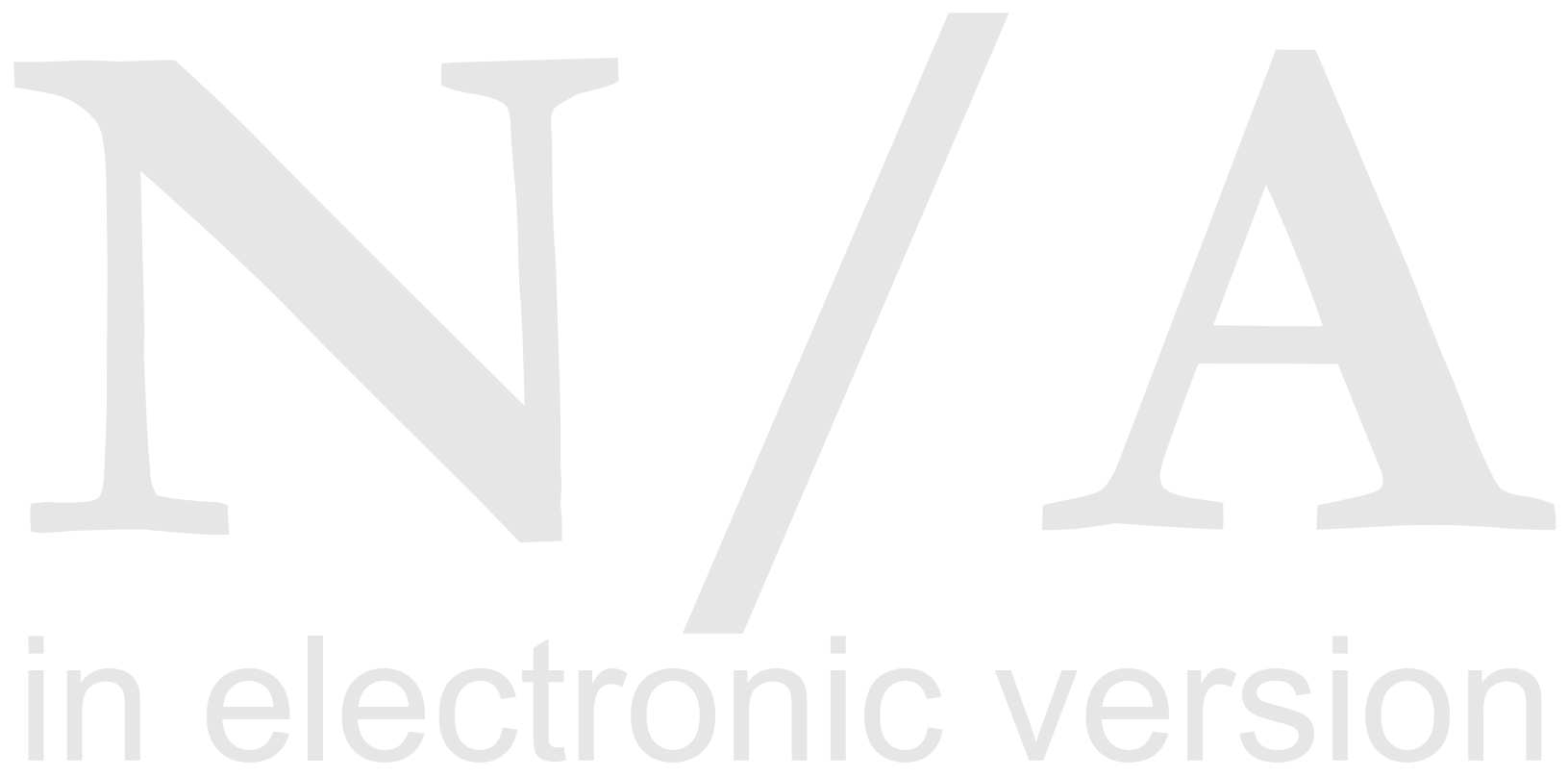


Towards Clinical Deployment of Automated Anatomical Regions-Of-Interest

(C) 2014 The Eurographics Association.

S. Lindholm, D. Forsberg, A. Ynnerman, H. Knutsson, M. Andersson, and C. Lundström. Towards clinical deployment of automated anatomical regions-of-interest. In Eurographics Workshop on Visual Computing for Biology and Medicine (VCBM), Vienna, Austria, 2014. The Eurographics Association, In press.

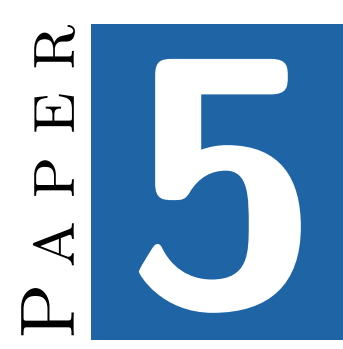




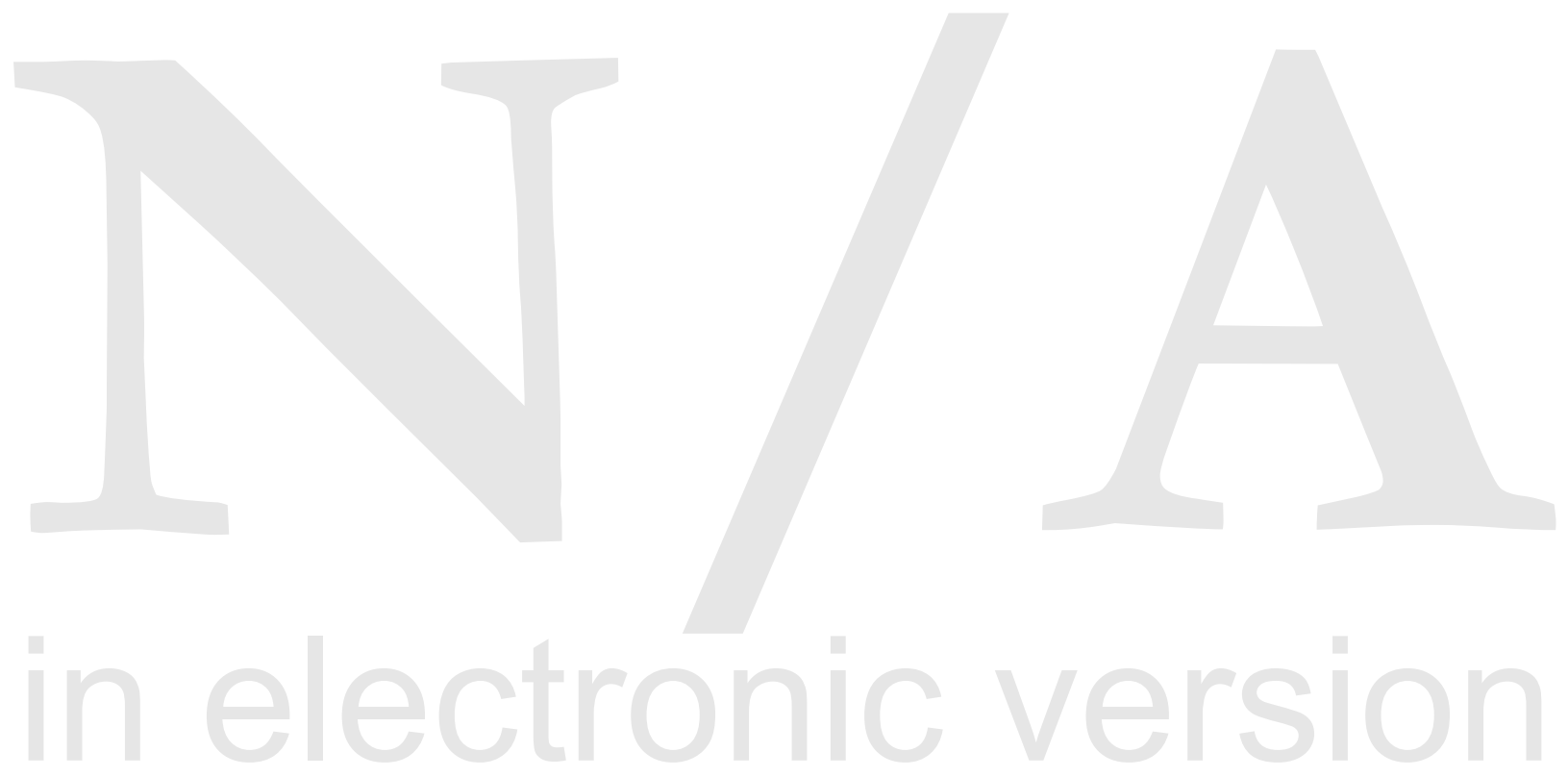




\section{Spatial Conditioning of Transfer Functions using Local Material Distributions \\ (C) 2010 IEEE Computer Society.}

S. Lindholm, P. Ljung, C. Lundström, A. Persson, and A. Ynnerman. Spatial conditioning of transfer functions using local material distributions. IEEE Transactions on Visualization and Computer Graphics, 16(6): 1301-1310, 2010. doi:10.1109/TVCG.2010.195.

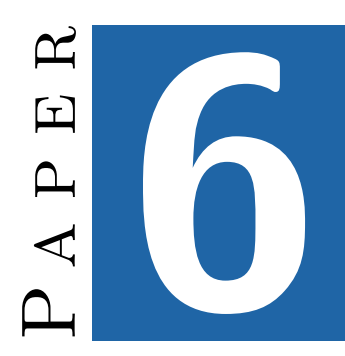




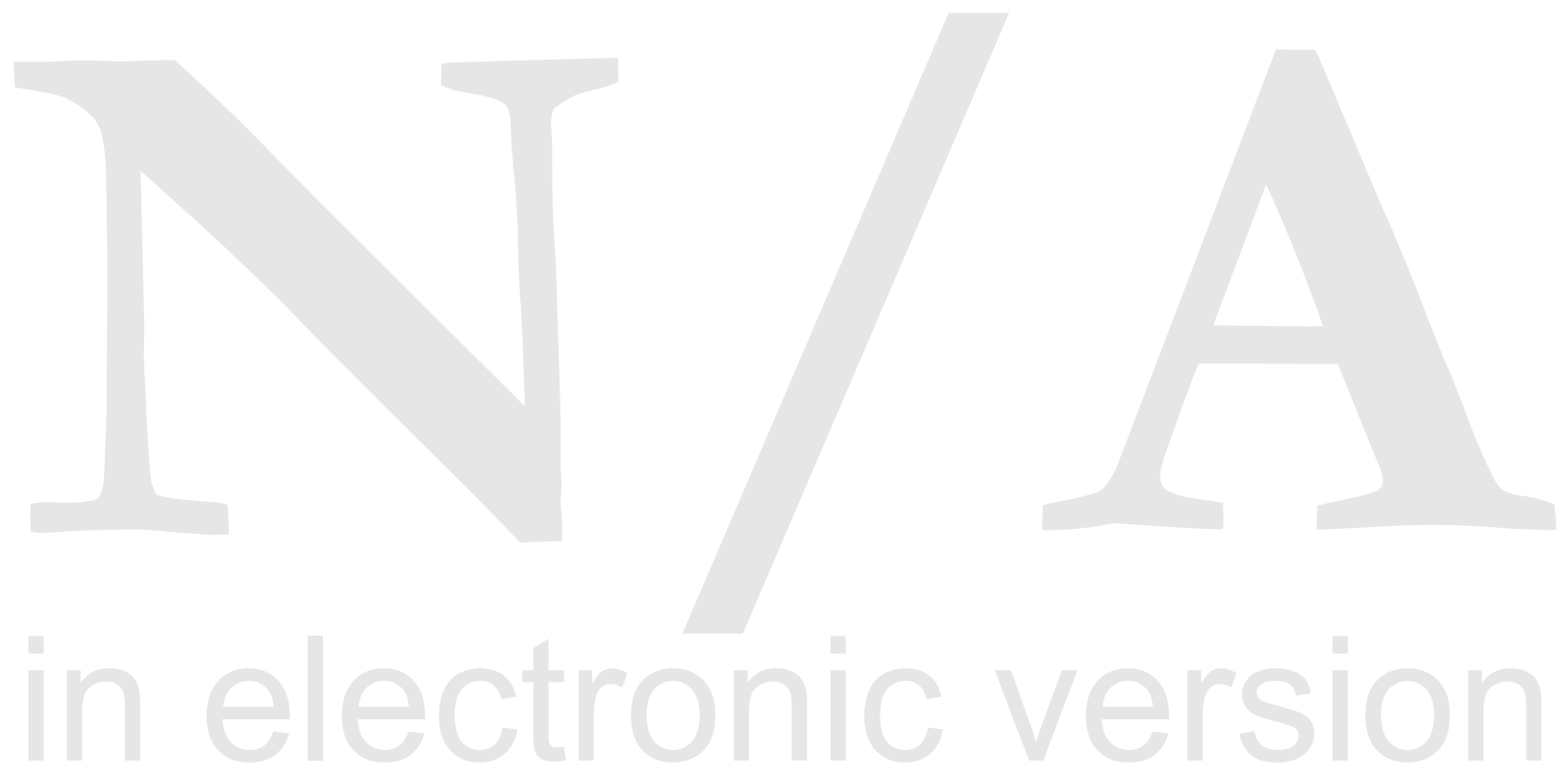




\section{Boundary Aware Reconstruction of Scalar Fields}

(C) 2014 IEEE Computer Society.

S. Lindholm, D. Jönsson, C. Hansen, and A. Ynnerman. Boundary aware reconstruction of scalar fields. IEEE Transactions on Visualization and Computer Graphics, 20(4), 2014. doi:10.1109/TVCG.2014.2346351, In press.

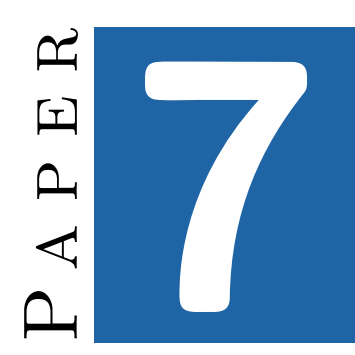




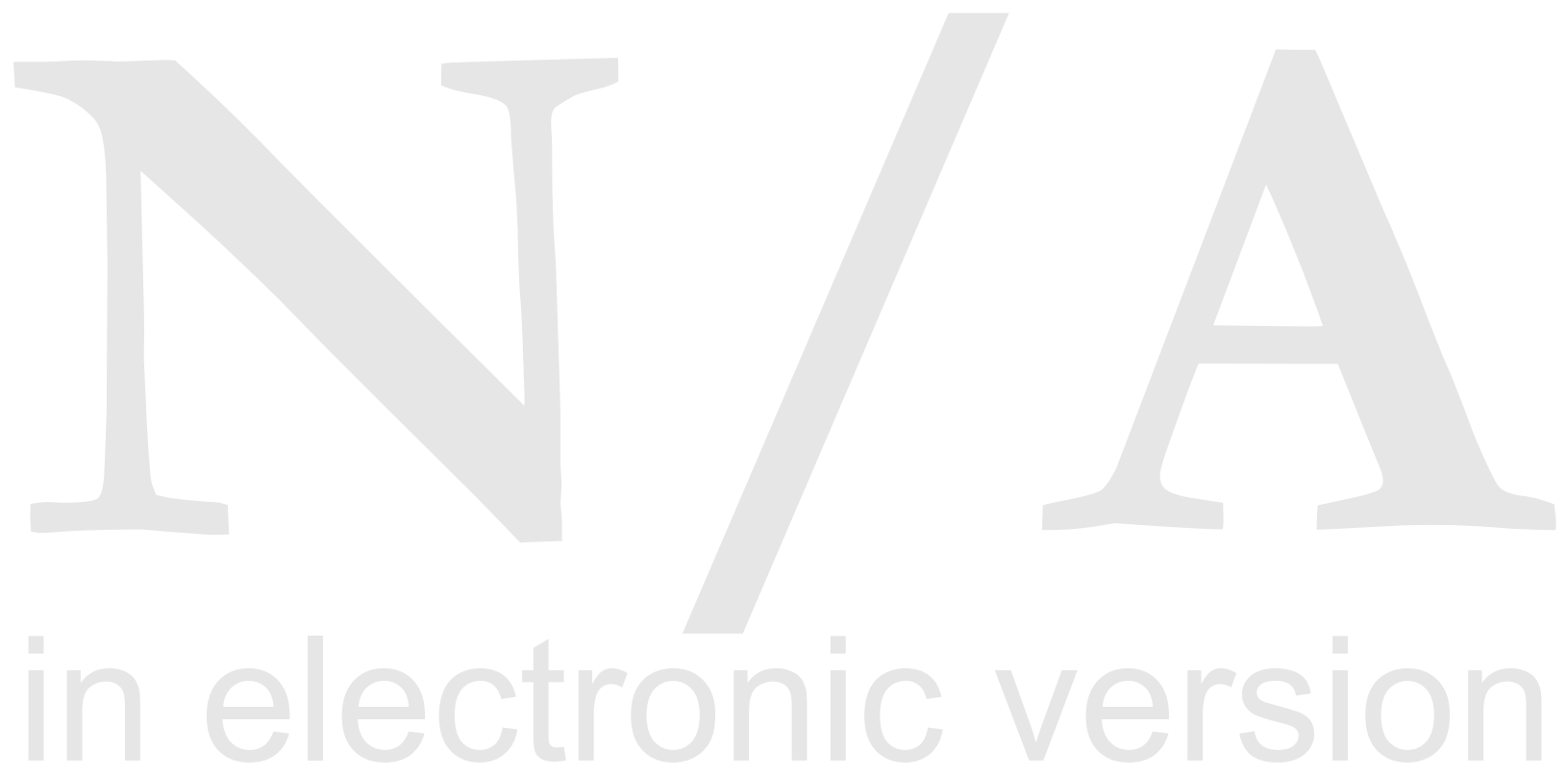

